\title{
Link-Layer Cooperative Communication in Vehicular Networks
}

by

\author{
Sailesh Bharati
}

\author{
A thesis \\ presented to the University of Waterloo \\ in fulfillment of the \\ thesis requirement for the degree of \\ Doctor of Philosophy \\ in \\ Electrical and Computer Engineering
}

Waterloo, Ontario, Canada, 2016

(C) Sailesh Bharati 2016 


\section{Author's Declaration}

I hereby declare that I am the sole author of this thesis. This is a true copy of the thesis, including any required final revisions, as accepted by my examiners.

I understand that my thesis may be made electronically available to the public. 


\begin{abstract}
Vehicular ad hoc networks (VANETs) are a special kind of communication networks and possess unique characteristics as compared with general mobile ad hoc networks (MANETs), where vehicles communicate with each other or with stationary road side units. Hence, directly applying the existing communication protocols designed for MANETs may not be reliable and efficient in VANETs. Thus, this thesis presents link-layer cooperative frameworks to improve transmission reliability and network throughput over distributed TDMA MAC ${ }^{1}$ protocols for VANETs.
\end{abstract}

We present a link-layer node cooperation scheme for VANETs, referred to as Cooperative ADHOC MAC (CAH-MAC). In CAH-MAC, neighboring nodes cooperate to utilize unused time slots to retransmit failed packets. Throughput improvement is achieved by using idle time slots that are wasted in the absence of node cooperation. In addition, as a packet is retransmitted earlier by a relay node, transmission delay and packet dropping rate are reduced. We study the effects of a dynamic networking environment on the performance of CAH-MAC. It is observed that, system performance degrades due to cooperation collisions. To tackle this challenge, we present an enhanced CAH-MAC (eCAH-MAC) scheme. In eCAH-MAC, using different types of packet and by delaying or suspending some relay transmissions, cooperation collisions can be avoided and cooperation opportunities can be efficiently utilize without disrupting the normal operations of the distributed TDMA MAC.

We propose a node cooperation based makeup strategy for vehicular networks, referred to as cooperative relay broadcasting (CRB), such that neighboring nodes proactively rebroadcast the packet from a source node. An optimization framework is developed to provide an upper bound on the CRB performance with accurate channel information. Further, we propose a channel prediction scheme based on a two-state first-order Markov chain, to select the best relaying node for CRB. As packets are repeatedly broadcasted by the neighboring nodes before they expire, the proposed CRB framework provides a more reliable broadcast service as compared with existing approaches.

The proposed node cooperation frameworks enhance the performance of distributed TDMA MAC and make it more robust to tackle VANET's dynamic networking conditions.

\footnotetext{
${ }^{1}$ Time division multiple access - medium access control.
} 



\section{Acknowledgements}

I would like to pay my sincere thanks to Professor Weihua Zhuang for her great supervision, constant guidance and support throughout my $\mathrm{PhD}$ study.

I would like to thank the members of my $\mathrm{PhD}$ thesis examining committee, Professor Mohamed Oussama Damen, Professor Nasser Lashgarian Azad, Professor Zhou Wang, and Professor Lian Zhao, for their valuable comments and suggestions, which help to improve the quality of this thesis. I would also like to thank Professor Guang Gong for her support to serve as a delegate during my $\mathrm{PhD}$ thesis defense.

I am grateful to Professor Xuemin (Sherman) Shen and BBCR group members, past and present, for their suggestions during my PhD study. Their constructive feedbacks and suggestions helped me to enjoy my PhD life at the BBCR lab.

Finally, I would like to express my deepest gratitude and am very thankful to my parents, my wife, Babita Yogi, and my brother, Santosh Bharati, for their endless support and love, continuous encouragement in my life, and helping me to be what I am today. 



\section{Dedication}

This PhD thesis is dedicated to my mother, Late Ambika Bharati, and father, Murari Prasad Bharati. 



\section{Table of Contents}

Author's Declaration

$\begin{array}{lll}\text { Abstracts } & \text { iii }\end{array}$

Acknowledgements $\quad$ v

Dedication $\quad$ vii

List of Tables $\quad$ xiii

List of Figures $\quad$ XV

List of Abbreviations $\quad$ xviii

List of Symbols $\quad$ xxi

1 Introduction $\quad 1$

1.1 Vehicular Ad-Hoc Networks . . . . . . . . . . . . . . . . 2

1.2 Medium Access Control in VANETs . . . . . . . . . . . . . . . 5

1.2.1 IEEE $802.11 \ldots \ldots \ldots \ldots \ldots$

1.2.2 Time Division Multiple Access . . . . . . . . . . . . . . . . . . 6 
1.3 Node Cooperation in Wireless Communication . . . . . . . . . . . 7

1.3.1 Link-Layer Cooperation . . . . . . . . . . . . . . . 7

1.3.2 Cooperation for Makeup Transmission . . . . . . . . . . . . 8

1.4 Motivation and Research Contributions . . . . . . . . . . . . . . . . 9

1.5 Organization of This Thesis . . . . . . . . . . . . . . . 11

2 System Model 12

2.1 Network Topology and Communication Among Nodes . . . . . . . . . . . . 12

2.2 Channel Access . . . . . . . . . . . . . . . . . . . . . 13

2.3 Time Synchronization Among Vehicles . . . . . . . . . . . . . . . 13

2.4 Neighboring Nodes . . . . . . . . . . . . . . . . . . . . . . . . . . . 14

2.5 Reservation and Retention of Time Slots . . . . . . . . . . . . . . . 15

3 Cooperation in ADHOC MAC $\quad 16$

3.1 Protocol Description . . . . . . . . . . . . . . . . . 17

3.1 .1 Frame Information . . . . . . . . . . . . . . . 18

3.1.2 Cooperation Among Neighboring Nodes . . . . . . . . . . . . . 19

3.1.3 Cooperation Header and Cooperation Acknowledgement . . . . . . 21

3.2 Preliminaries for the Performance Analysis . . . . . . . . . . . . . . . . . 23

3.2.1 Node Distribution . . . . . . . . . . . . . . . . 23

3.2.2 Distribution of the Neighboring Node Numbers . . . . . . . . . . . 24

3.2.3 Transmission During a Time Slot . . . . . . . . . . . . . 25

3.2.4 Types of Time Slots and Their Distributions . . . . . . . . . . 26

3.2.5 Cooperation Enabled Transmission . . . . . . . . . . . . 27

3.2.6 Benefit of Cooperation . . . . . . . . . . . . . . . . . 29 
3.3 Throughput Analysis . . . . . . . . . . . . . . . . . . . . . . . . . . . . . 29

3.3.1 Expected Number of Successful Time Slots . . . . . . . . . . . . 29

3.3 .2 Throughput and Throughput Gain . . . . . . . . . . . . . 30

3.4 Reliability Analysis . . . . . . . . . . . . . . . . . . . . . 31

3.4 .1 Packet Transmission Delay . . . . . . . . . . . . . . . . . . 31

$3.4 .2 \quad$ Packet Dropping Rate $\ldots \ldots \ldots \ldots$

3.5 Numerical Results . . . . . . . . . . . . . . . . . . . . . . . . . 33

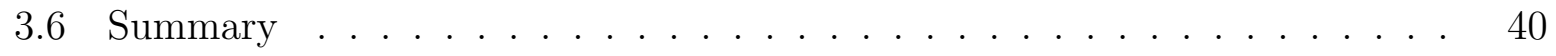

4 Enhanced Node Cooperation $\quad 41$

4.1 System Overview . . . . . . . . . . . . . . . . . . . . . . . . . 42

4.1 .1 Channel Model . . . . . . . . . . . . . . . . . . . . . . . . 42

4.1.2 Cooperation Collisions and Release of Time Slots . . . . . . . . 44

4.2 Enhanced Cooperative ADHOC MAC . . . . . . . . . . . . . . 45

4.2 .1 Types of Packet Structure . . . . . . . . . . . . . . . 45

4.2 .2 Cooperation Collision Avoidance . . . . . . . . . . . . 47

4.3 Utilization of an Unreserved Time Slot . . . . . . . . . . . . . . 49

4.3.1 Distribution of Node Number . . . . . . . . . . . . . . . . 51

4.3.2 Probability of Event $3 \ldots \ldots \ldots \ldots$

4.3 .3 Probability of Event $4 \ldots \ldots \ldots \ldots \ldots$

4.3.4 Close-Form Expressions for Time Slot Utilization . . . . . . . 54

4.4 Numerical Results . . . . . . . . . . . . . . . . . . . . . . 55

4.5 Simulation Results . . . . . . . . . . . . . . . . . 57

4.6 Summary . . . . . . . . . . . . . . . . . . . . . . . 62 
5 Cooperative Relay Broadcasting $\quad 65$

5.1 Node Cooperation for Broadcast Service in D-TDMA MAC . . . . . . . . 67

5.2 Optimal Helper Selection with Accurate Channel Information . . . . . . . 69

5.3 Helper Selection with Channel Prediction . . . . . . . . . . . . 71

5.3.1 Prediction of Failed and Successful Nodes . . . . . . . . . . . . 72

5.3.2 Cooperation Decisions . . . . . . . . . . . . . . 74

5.3.3 Cooperative Relay Broadcasting . . . . . . . . . . . . . . . 74

5.4 Simulation Results . . . . . . . . . . . . . . . . 76

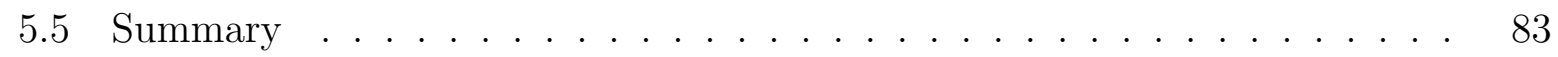

6 Conclusions and Future Works $\quad 84$

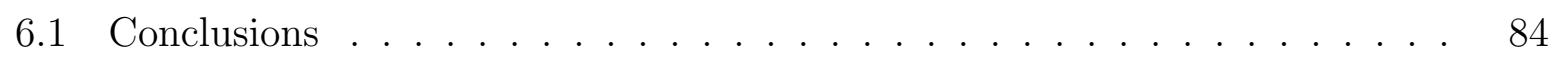

6.2 Future Research Directions . . . . . . . . . . . . . . . . 86

$\begin{array}{lr}\text { References } & 89\end{array}$

Appendix A Multi-Channel Operation in VANETs 99

$\begin{array}{lll}\text { Appendix B } & \text { Two-state Markov Channel } & 101\end{array}$ 


\section{List of Tables}

4.1 Parameters used in simulation . . . . . . . . . . . . . . 55

4.2 VISSIM simulation parameters . . . . . . . . . . . . . . . . . 58

4.3 Parameters for doppler spread in different driving environments[1] . . . . 59 



\section{List of Figures}

1.1 An illustrative network architecture of a VANET. . . . . . . . . . . . . 2

2.1 Use of the 1PPS signal for time synchronization. . . . . . . . . . . . 13

2.2 Illustration of a two-hop set with node $A$ as a reference, where an ellipse represents an OHS such that all nodes inside one ellipse can directly communicate with each other. . . . . . . . . . . . . . . . . . . . 14

3.1 Packet structure in CAH-MAC . . . . . . . . . . . . . . . 18

3.2 Structure of a frame information field, where $\phi$ indicates an empty field. . . 18

3.3 Information exchanges in the CAH-MAC: (a) Phase 1: Source node fails to transmit a packet to the destination; (b) Phase 2: Neighboring nodes detect transmission failure after examining the FI from the destination; (c) Phase 3: Helper node $H$ offers cooperation; (d) Phase 4: Helper node $H$ re-transmits the packet that failed to reach the destination after receiving a cooperation acknowledgement from the destination. . . . . . . . . . . . . . 21

3.4 Cooperative relay transmission during an unreserved time slot. . . . . . . 22

3.5 Illustration of the common coverage road segment of an $s-d$ pair. . . . . 24

3.6 Throughput comparison of ADHOC MAC and CAH-MAC with $R=300 \mathrm{~m}$

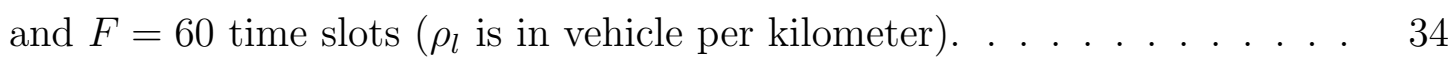

3.7 Throughput comparison of ADHOC MAC and CAH-MAC with $\rho_{l}=30$ vehicle per kilometer and $F=60$ time slots. . . . . . . . . . . . 34 
3.8 Throughput comparison of ADHOC MAC and CAH-MAC with $\rho_{l}=30$ vehicle per kilometer and $R=300 \mathrm{~m}$. . . . . . . . . . . . . .

3.9 Throughput gain of CAH MAC over ADHOC MAC with $F=60$ time slots

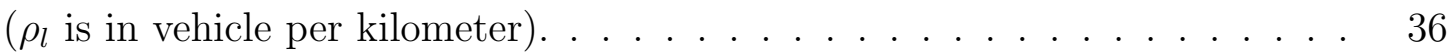

3.10 Packet transmission delay of ADHOC MAC and CAH-MAC with $\rho_{l}=50$ vehicle per kilometer and $F=60$ time slots. . . . . . . . . . . .

3.11 Packet dropping rate of ADHOC MAC and CAH-MAC with $M_{\max }=1$ frame, $\rho_{l}=30$ vehicle per kilometer and $F=60$ time slots. . . . . . . . . 38

3.12 Packet dropping rate of ADHOC MAC and CAH-MAC with $M_{\max }=5$ frames, $\rho_{l}=30$ vehicle per kilometer and $F=60$ time slots. . . . . . . . .

3.13 Packet dropping rate of ADHOC MAC and CAH-MAC with $M_{\max }=10$ frames, $\rho_{l}=30$ vehicle per kilometer and $F=60$ time slots. . . . . . . . .

4.1 Cooperative relay transmission in eCAH-MAC during an unreserved time slot. 47

4.2 Suspension of a cooperative relay transmission by the helper node after failing to receive C-ACK. . . . . . . . . . . . . . . . . 48

4.3 Suspension of a cooperative relay transmission by the destination node in the presence of the contending node(s) in its one-hop distance. . . . . . . .

4.4 Comparison of utilization of unreserved time slots in eCAH-MAC, CAH-MAC and ADHOC MAC with (a) $\eta=0.80$; (b) $\eta=0.50$; (c) $\eta=0.20 \ldots$. . . .

4.5 Status of two-hop member nodes and time slots of the corresponding frame with $\aleph=622$ over various channel conditions: (a) Portion of the number of resident and contending nodes in a THS per frame; (b) Portion of reserved and unreserved time slots in a THS per frame; (c) Portion of reserved and unreserved time slots in a THS per frame observed between consecutive frames in a steady state. . . . . . . . . . . . . . . . . . . . 
4.6 Probability of events during cooperative relay transmission (CRT), such as successful CRT (Successful), failed CRT due to channel error (Channel error), and failed CRT due to cooperation collision in CAH-MAC or suspension of CRT to avoid cooperation collision in eCAH-MAC (Coop. coll/susp.), with (a) $\alpha=2$; (b) $\alpha=3$; (c) $\alpha=4 \ldots \ldots \ldots \ldots$

4.7 Probability of successful usage of unreserved time slots in ADHOC MAC, CAH-MAC and eCAH-MAC, with (a) $\alpha=2$; (b) $\alpha=3$; (c) $\alpha=4 \ldots \ldots$.

5.1 CRB performed by the best helper node and suspension of CRB(s) from the other potential helper node(s) that is (are) not within the one-hop distance from the transmitting helper node. . . . . . . . . . . . . . . . 76

5.2 Packet received rate in D-TDMA MAC, CRB-OPT and CRB-HSCP with (a) $\alpha=2$ and 3 ; (b) $\alpha=4 \ldots \ldots \ldots \ldots \ldots \ldots \ldots$

5.3 Packet delivery ratio with $\alpha=3$ in D-TDMA MAC, CRB-OPT and CRBHSCP such that (a) 50\%; (b) $75 \%$; (c) $99 \%$ of nodes in the area of interest received packet within a duration of one time frame. . . . . . . . . . . .

5.4 Packet delivery ratio with $\alpha=4$ in D-TDMA MAC, CRB-OPT and CRBHSCP such that (a) $50 \%$; (b) $75 \%$; (c) $99 \%$ of nodes in the area of interest received packet within a duration of one time frame. . . . . . . . .

5.5 Normalized number of retransmission attempts over the number of unreserved time slot in CRB-OPT and CRB-HSCP with (a) $\alpha=3$; (b) $\alpha=4 \ldots \ldots$.

A.1 Spectrum and Power Allocation for DSRC. . . . . . . . . . . . . . 99

B.1 Two-state Markov chain channel. . . . . . . . . . . . . . . . . . . . . 102 


\title{
List of Abbreviations
}

\author{
$s-d \quad$ A pair of source and destination nodes \\ 1PPS One-Pulse-Per-Second \\ ACK Acknowledgement \\ AU Application Unit \\ C-ACK Cooperation Acknowledgement \\ CAH-MAC Cooperative ADHOC MAC \\ $\mathrm{CCH} \quad$ Control Channel \\ cdf Cumulative Density Function \\ CDMA Code Division Multiple Access \\ $\mathrm{COH} \quad$ Cooperation Header \\ CRB-HSCP Cooperative Relay Broadcasting with Helper Selection Based on Channel \\ Prediction \\ CRB-OPT Cooperative Relay Broadcasting with Optimal Helper Selection \\ CRC Cyclic Redundancy Check \\ CRT Cooperative Relay Transmission \\ xviii
}




\begin{tabular}{ll} 
D-DTMA & Distributed Time Division Multiple Access \\
DIFS & Distributed Inter-frame Space \\
DSRC & Dedicated Short Range Communication \\
eCAH-MAC & enhanced Cooperative ADHOC MAC \\
EDCA & Enhance Distributed Channel Access \\
FCC & Federal Communication Commission \\
FI & Frame Information \\
GPS & Global Positioning System \\
HGV & Heavy Goods Vehicle \\
i.i.d & Independent and Identically Distributed \\
IDF & Identification Field \\
ITS & Intelligent Transportation Systems \\
MAC & Medium Access Control \\
MANET & Mobile Ad Hoc Network \\
MSD & Minimum Safety Distance \\
NACK & Negative Acknowledgement \\
OBU & On-board Unit \\
OHS & One-Hop Set \\
Pdf & Probability Density Function \\
PH & Packet Header \\
\hline
\end{tabular}

xix 


$\begin{array}{ll}\text { pmf } & \text { Probability Mass Function } \\ \text { PN } & \text { Pseudo-random Noise } \\ \text { QoS } & \text { Quality-of-Service } \\ \text { RSU } & \text { Road Side Unit } \\ \text { SCH } & \text { Service Channel } \\ \text { SDMA } & \text { Space Division Multiple Access } \\ \text { TDMA } & \text { Time Division Multiple Access } \\ \text { THS } & \text { Two-Hop Set } \\ \text { Type-C } & \text { Cooperation Packet } \\ \text { Type-G } & \text { General Packet } \\ \text { Type-R } & \text { Reservation Packet } \\ \text { V2I } & \text { Vehicle-to-Infrastructure } \\ \text { V2V } & \text { Vehicle-to-Vehicle } \\ \text { VANET } & \text { Vehicular Ad Hoc Network } \\ \text { WAVE } & \text { Wireless Access in Vehicular Environment } \\ \text { WBSS } & \text { Wave-Mode Basic Service Set } \\ \text { WSA } & \text { WAVE Service Advertisement }\end{array}$




\title{
List of Symbols
}

\author{
$A C 0 \quad$ Access Catagory 0 \\ $A C 1 \quad$ Access Catagory 1 \\ $A C 2$ Access Catagory 2 \\ AC3 Access Catagory 3 \\ $E_{1} \quad$ Event that potential helpers exist for cooperative relay transmission \\ $E_{2} \quad$ Event that unreserved time slots exist for cooperative relay transmission \\ $E_{3} \quad$ Event that a contending node chooses an unreserved time slot among all the \\ available unreserved time slots \\ $E_{4} \quad$ Event that an unreserved time slot is not selected by contending nodes \\ $F \quad$ The number of time slots per frame \\ $G_{r} \quad$ Antenna gain at a receiving node \\ $G_{t} \quad$ Antenna gain at a transmitting node \\ $I_{v}(\cdot) \quad$ The $v^{t h}$ order modified Bessel function of the first kind \\ $J_{0}(\cdot) \quad$ The zeroth-order Bessel function of the first kind \\ $L \quad$ The number of lanes in a road-segment \\ $M \quad$ The packet transmission delay, in frames, in ADHOC MAC \\ $M_{\text {coop }}$ The packet transmission delay, in frames, in CAH-MAC
}


$M_{\max } \quad$ The maximum packet transmission limit

$N_{C} \quad$ The number of contending nodes in a reference two-hop neighborhood

$N_{T}\left(\bar{N}_{T}\right) \quad$ The (average) number of nodes in a reference two-hop neighborhood

$N_{o} \quad$ The number of common one-hop neighbors of a pair of source and destination nodes

PDR The packet dropping rate in ADHOC MAC

$P D R_{\text {coop }}$ The packet dropping rate in CAH MAC

$P_{t} \quad$ The constant transmission power of all nodes

$P_{b b} \quad$ The probability that channel is in the bad state in the current time slot and remains unchanged in the next time slot

$P_{g g} \quad$ The probability that channel is in the good state in the current time slot and remains unchanged in the next time slot

$R \quad$ Transmission range of a vehicle

$T \quad$ The number of frames per second

$U \quad$ The number of failed time slots per frame

$X \quad$ The number of successful time slots per frame

$X_{\text {coop }} \quad$ The number of sucessful time slots in per frame with cooperation enabled transmission

$Y \quad$ The number of potential helpers for a given failed packet transmission

$\Delta \quad$ A fixed, and small, time duration

$\Gamma(\cdot) \quad$ The gamma function

$\Gamma(\cdot, \cdot) \quad$ The upper incomplete Gamma function

$\Phi_{3}(\cdot, \cdot ; \cdot, \cdot) \quad$ The confluent hypergeometric function 
$\Pi \quad$ The set of calculated probability of finding the channel in a good state

$\aleph \quad$ The number of vehicles in the network

$\alpha \quad$ Path-loss exponent

$\beta \quad$ The sum of time durations to sense the channel, transmission time of a cooperative acknowledgement, and the guard time

$\beta_{1} \quad$ The time duration during which a destination node sense the channel before cooperative relay transmission

$\beta_{2} \quad$ The transmission time of a cooperative acknowledgement plus the guard time

$\delta \quad$ A fixed number such that $\delta \Delta$ is the time durations of a time slot minus the transmission time to perform cooperative relay broadcasting

$\delta_{z} \quad$ A random number that node $z$ draws for black-burst before cooperative relay broadcasting

$\epsilon_{A} \quad$ The utilization of an unreserved time slot in ADHOC MAC

$\epsilon_{C} \quad$ The utilization of an unreserved time slot in CAH-MAC

$\epsilon_{E} \quad$ The utilization of an unreserved time slot in eCAH-MAC

$\eta \quad$ The ratio of the number of reserved time slots in a frame to the total number of time slots per frame

$\gamma_{r}\left(\bar{\gamma}_{r}\right)$ The (average) received power by a node at a distance $r$ from a transmitter

$\gamma_{t h} \quad$ Threshold received power level

$\mathcal{A}_{z} \quad$ The set of failed nodes, predicted and reported combined, from the perspective of node $z$

$\mathcal{B}_{x} \quad$ The set all time slots which belong to two-hop neighbors of node $x$ $\mathcal{D}^{\prime} \quad$ The optimal set of potential destination nodes that received packet during a cooperative relay broadcasting 
$\mathcal{D}$ The set of potential destination nodes that do not have the tagged packet

$\mathcal{F} \quad$ The set of all time slots in a frame

$\mathcal{H}$ The set of potential helper nodes with the tagged packet

$\mathcal{K}$ The set of indicator variables $\left(k_{x y}\right)$ indicating the transmission status from node $y$ to node $x$

$\mathcal{M}_{i} \quad$ A random process representing the channel state during $i^{\text {th }}$ time slot

$\mathcal{O}_{z} \quad$ The set of all one-hop neighbors of node $z$

$\mathcal{O}_{z}^{f} \quad$ The set of one-hop neighbors of node $z$ that failed to receive the tagged packet

$\mathcal{O}_{z}^{r} \quad$ The set of one-hop neighbors of node $z$ that already reported its transmission status with a source node

$\mathcal{O}_{z}^{s} \quad$ The set of one-hop neighbors of node $z$ that successfully received the tagged packet

$\mathcal{O}_{z}^{c r b}$ The set of one-hop neighbors of node $z$ that already performed cooperative relay broadcasting

$\mathcal{P}_{z}^{f} \quad$ The predicted set of one-hop neighbors of node $z$ that failed to receive the tagged packet

$\mathcal{P}_{z}^{s} \quad$ A predicted set of one-hop neighbors of node $z$ that sucessfully received the tagged packet

$\mathcal{Q}$ The set of indicator variables $\left(q_{y}\right)$ indicating the selection of node $y$ as the best potential helper node

$\mathcal{R}_{z}^{f} \quad$ The set of one-hop neighbors of node $z$ that reported failure to receive the tagged packet

$\mathcal{R}_{z}^{s} \quad$ A set of one-hop neighbors of node $z$ that reported the successful reception of the tagged packet 
$\mathcal{T}_{z} \quad$ The set of all two-hop neighbors of node $z$

$\mathcal{V} \quad$ The set of indicator variables $\left(v_{x y}\right)$ indicating the channel condition from node $y$ to node $x$

$\pi_{g} \quad$ The steady state probability of finding the channel in the good state

$\pi_{g}^{x y} \quad$ The steady state probability of finding the channel in the good state when node $x$ transmits a packet to receiving node $y$

$\rho \quad$ The vehicle density of a road-segment

$\rho_{i} \quad$ The vehicle density of lane $i$

$\sigma \quad$ The throughput of ADHOC MAC

$\sigma_{\text {coop }}$ The throughput of CAH-MAC

$\sigma_{\text {gain }}$ The normalized throughput gain achieved by cooperation in CAH-MAC over ADHOC MAC

$\tau \quad$ The time duration of a time slot

$\theta \quad$ The slope value of effective velocity

$\varrho \quad$ The amplitude correlation coefficient of a received signal

c The speed of light

$f_{c} \quad$ The carrier frequency of signals

$f_{d} \quad$ The average doppler spread

$m$ The shape parameter of the Nakagami- $m$ channel

$o \quad$ The offset value of effective velocity

$p \quad$ The probablity that a vehicle within the transmission range of a source node successfully receive the transmitted packet, taking account of a possible poor channel condition 
$p(n, z)$ The probablity of finding $m$ vehicles along a road segment of length $n$

$p_{c} \quad$ The probablity of transmission collision in a given time slot

$p_{s} \quad$ The probability of successful transmission between a pair of source and destination nodes during a reserved time slot

$p_{s}^{c o o p}$ The probability of successful transmission between a pair of source and destination nodes during a reserved time slot, with cooperation enabled transmission

$p_{\text {coop }} \quad$ The probablity of cooperation

$v_{r} \quad$ The velocity of a receiver

$v_{t} \quad$ The velocity of a transmitter

$v_{\text {eff }} \quad$ The effective velocity between receiver and transmitter

$w_{l} \quad$ The width of (each) lane $l$ of the road 


\section{Chapter 1}

\section{Introduction}

Increasing road accidents and vehicle traffic congestions have led to the evolution of intelligent transportation systems (ITS) [2] and other applications that improve road safety, increase transportation efficiency, and provide on-board infotainment. To make these applications possible, vehicles are equipped with sensors and communication devices such that they can gather and exchange information to maintain road safety as well as to optimize vehicle-traffic efficiency. Moreover, wireless technology makes communication among vehicles possible, forming a vehicular ad hoc network (VANET). Due to the rapid advancement in the wireless communication technology and automotive industries, the paradigm of VANETs emerges as a promising approach to provide a communication infrastructure supporting ITS and other on-board applications, primarily to improve road safety. The National Highway Traffic Safety Administration (NHTSA) of the United States Department of Transportation (USDoT) has predicted that traffic accidents, specifically vehicle collisions, can be reduced by approximately $80 \%$ through the deployment of safety applications enabled by VANETs [3]. In addition, comfort related applications such as gaming, automatic toll collection, drive-thru Internet connections are expected to be delivered to vehicles for their drivers and passengers, providing on-board infotainment services [4].

Motivated by its immense potential, several stake holders including governments, academia, and automotive industries have taken initiatives in developing VANETs, with various projects $[5,6,7,8,9,10]$ focusing on designing protocols, algorithms, and systems 


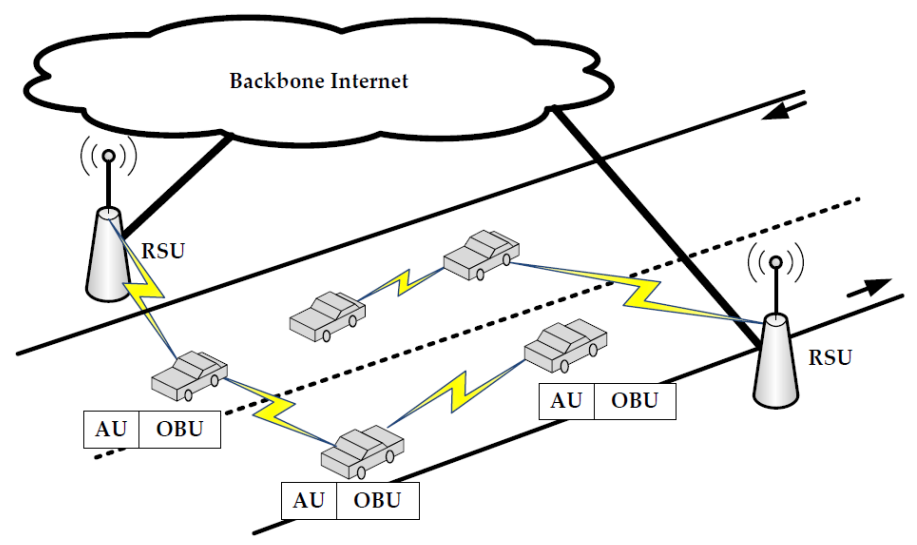

Figure 1.1: An illustrative network architecture of a VANET.

for VANETs. In addition, several consortiums [11, 12] have been established to standardize the proposed protocols and algorithms. Furthermore, government agencies have come forward and allocated radio spectrum for VANETs. In the United State, the Federal Communications Commission (FCC) has allocated $75 \mathrm{MHz}$ of Dedicated Short Range Communication (DSRC) spectrum in $5.9 \mathrm{GHz}$ band [13]. As this band is not available in Europe, two $10 \mathrm{MHz}$ radio spectrum bands have been proposed in $5.9 \mathrm{GHz}, 5.885$ $5.895 \mathrm{GHz}$ for control signal and safety applications and $5.865-5.875 \mathrm{GHz}$ for non-safety applications [13].

\subsection{Vehicular Ad-Hoc Networks}

\section{VANET Architecture}

VANETs are a special kind of mobile ad hoc networks (MANETs) in which vehicles are primary communication nodes, to facilitate a safe and efficient vehicle transportation system. In contrast to MANETs, VANETs may use the fixed stations that are deployed along the roadside. Fig. 1.1 illustrates a simple network architecture of VANETs, where vehicles on the road can communicate with each other and/or with a fixed station along the roadside. Each vehicle is equipped with an on-board unit (OBU) and/or one or multiple application units (AUs) [14]. The OBU, a device with wireless networking interfaces, enables vehicles 
to communicate. The AUs, on the other hand, are devices which run application(s) and make use of the OBU to exchange information with other vehicles.

Vehicles communicate independently either with each other or with stationary road side units (RSUs) which can be traffic light units, roadside monitors, and information traffic gateways connected to the Internet. Thus, VANETs will support both vehicle-to-vehicle (V2V) and vehicle-to-infrastructure (V2I) communications [13].

\section{VANET Applications}

Vehicles communicate via a radio channel to exchange messages to support a wide range of mobile distributed applications. Based on their objectives, these applications can be broadly categorized into the following three groups $[13,15,16,17,18]$.

1. Safety applications to improve road safety: Providing warning messages to drivers regarding safety threats, such as due to possible vehicles collision, road damage, weather condition, is an example of safety applications;

2. Traffic management applications to increase the vehicle traffic efficiency: Navigating to an alternative route in case of a disaster or heavy traffic condition avoiding congestions, assisting a driver to find parking space, are examples of traffic management applications;

3. Infotainment applications to entertain or comfort drivers and/or passengers: Invehicle internet access, online gaming, video streaming, point-of-interest information, location-specific information/advertisements, and electronic flyer distribution can be considered under this category.

\section{VANET Characteristics}

VANETs have some unique characteristics as compared to other forms of wireless networks such as MANETs or cellular networks. The differences are mainly in terms of the network architecture (presence of stationary RSUs and highly mobile vehicles), users mobility pattern (presence of highly mobile vehicles with restricted mobility guided by the geometry 
of roadways), energy constraints (presence of high capacity on-board batteries) and, finally, the presence of real-life applications [19]. Furthermore, the radio spectrum allocated for VANETs is divided into multiple channels as in DSRC, such that the control channel is used for broadcasting safety messages and control information, and negotiation between a pair of provider and user to run infotainment applications using the service channels (spectrum allocation of DSRC and multi-channel operations are discussed in Appendix A). Such differences result in both challenges and opportunities in the development and operation of VANETs. For example, the on-board batteries are capable of supplying sufficient energy and alleviate energy constraints on VANET applications and communication protocols. On the other hand, high node mobility and variations in vehicle speed and density in space (such as city, highway, and rural areas) and in time (such as holidays, rush hours) result in dynamic topology changes with frequent link breakage [20,21, 22]. Moreover, a wide range of VANET applications imposes diverse quality-of-service (QoS) requirements in its communication protocols. For example, most of the safety applications require a message ${ }^{1}$ to reach to $99 \%$ of the target destination nodes within 100 milliseconds [18, 23, 24], i.e., high communication reliability and strict delay constraints $[15,25]$, which is difficult to guarantee due to the VANET's dynamic networking conditions. A broadcast service supports disseminating messages from a source node generating the messages to its neighboring nodes [26]. Consequently, safety applications use the broadcast service for disseminating messages to nodes within an area of interest, e.g., within one-hop transmission distance of a source node generating the messages. Hence, an efficient, quick, and reliable broadcast service is required to successfully deploy the safety applications with stringent QoS requirements. On the other hand, non-safety applications such as gaming and video streaming are throughput sensitive.

In order to develop a robust, efficient, and reliable communication protocol in VANETs, the aforementioned advantages should be exploited and the challenges must be addressed. One of possible ways to meet such challenges is by enabling node cooperation focusing on link-layer or medium access control (MAC) protocol design in VANETs.

\footnotetext{
${ }^{1}$ Safety messages can be either periodic or event-driven [18]. Periodic messages are generated periodically, normally 10 messages per second, and consist of information such as position, speed, deceleration, etc. On the other hand, event-driven messages are generated when some undesired events occur, such as bad road condition, sudden lane changing, etc., to warn the nearby drivers about the events.
} 


\subsection{Medium Access Control in VANETs}

Several approaches have been considered while designing a MAC protocol for VANETs. Such MAC protocols are designed based on channel access schemes such as code division multiple access (CDMA), space division multiple access (SDMA), IEEE 802.11 random access, and time division multiple access (TDMA). MAC protocols based on CDMA channel access $[27,28]$ require each vehicle to have a database of allowable location-specific pseudo-random noise $(\mathrm{PN})$ codes specified in its digital map. In addition, vehicles must be equipped with a large number of match filters, which depends on the length of PN codes. Similarly, MAC protocols based on SDMA channel access [29, 30] require each vehicle to have a location specific channel-allocation database in its digital map. These factors add complexity in the protocol development and make protocols difficult to implement. Hence, in the following, we focus only on MAC protocols based on IEEE 802.11 and TDMA channel access schemes, which are widely used in research studies related to VANETs.

\subsubsection{IEEE 802.11}

The IEEE 802.11 random access scheme is a well known MAC protocol in wireless networks [31]. Such a scheme allows a node to contend for the channel and access it if it is found idle. Variations in the IEEE 802.11 standard are made to make it suitable for a high mobility scenario, which is highly likely in VANETs. The IEEE 802.11p standard [32] is developed for VANETs, which targets to support vehicle-to-vehicle (V2V) and vehicle-to-infrastructure (V2I) communication. MAC protocols based on the IEEE 802.11p standard also support QoS provision and is governed by the IEEE 802.11e enhance distributed channel access (EDCA) standard [33], such that traffic packets are differentiated into four different access categories, $A C 0-A C 3$, where $A C 0$ has the lowest priority and $A C 3$ has the highest priority to access the channel. Service differentiation is achieved in terms of a back-off interval while accessing channel, such that a high priority traffic packet, in general, experiences a shorter back-off interval than that of a low priority packet. Although the IEEE 802.11p access scheme is considered in several studies to develop MAC protocols for VANETs, it suffers from unbounded latency [34] and broadcast storm [35, 36]. Moreover, in the IEEE 802.11p MAC protocol, even successful broadcast packets are left unacknowledged. This 
results in an unreliable broadcast service. Since broadcast service is critical to support safety applications, MAC protocols based on the IEEE 802.11p standard may not be reliable enough for VANETs. In VANETs, the high priority safety messages are short range, uncoordinated, and broadcast in nature [37]. They have a strict delay requirement and demand a reliable broadcast service. However, due to the aforementioned limitations, MAC protocols based on the IEEE 802.11 are not suitable to provide the required QoS in VANETs.

\subsubsection{Time Division Multiple Access}

In [35], the ADHOC MAC, a distributed time division multiple access (TDMA) based MAC protocol, abbreviated as D-TDMA MAC, is proposed for VANETs. The ADHOC MAC is based on RR-ALOHA [38] and supports reliable multi-hops, point-to-point, and broadcast services. The channel time is divided into time frames and each frame is further divided into a fixed number of time slots. Nodes contend for time slots. A single time slot is used by only one node within a two-hop transmission distance. Since each node, within the two-hop transmission distance, uses an unique time slot, the hidden node terminal problem is solved. In addition, the broadcast storm problem $[35,36]$ is solved as a node rebroadcasts a packet only when needed, i.e., if it did not sense retransmission from any of its neighboring nodes. In [39], the authors show that the network throughput reduces due to the mobility among nodes. Under such a mobile scenario, transmission collisions among vehicles moving in opposite directions or between vehicles and the stationary road side units (RSUs) increase, reducing the throughput. VeMAC is proposed in [40] to minimize the throughput reduction due to the relative mobility as well as to provide a reliable broadcast service. Moreover, D-TDMA MAC protocols are reliable with a small chance of transmission collisions and have explicit ACKs for each transmitted messages, which the IEEE 802.11p fails to provide [41]. However, due to the VANET dynamic topology, the D-TDMA MAC protocols may lead to wastage of time slots. The wastage occurs when there are not enough nodes in the neighborhood to use all the available time slots of a frame. In addition, upon a transmission failure, the source node has to wait until the next frame for retransmission even if the channel is idle during unreserved time slots. 


\subsection{Node Cooperation in Wireless Communication}

Wireless networks are affected by various channel impairments (such as fading, shadowing, path loss) and limited radio spectrum resources. Various techniques such as diversity and channel coding exist in link-layer protocols to mitigate wireless channel impairments and to improve network throughput. However, these techniques may introduce some overhead or require multiple antennas and/or transceivers. An alternative approach is cooperative communication, which makes use of nearby nodes to improve transmission performance between a pair of source and destination $(s-d)$ nodes via diversity gain. The broadcast nature of a wireless transmission enables neighboring nodes to overhear the transmission of a packet from the source node to the destination. When the direct transmission between the $s-d$ pair suffers from a poor channel condition, the overheard packet can be relayed to the destination node by a node or nodes which have a good channel condition to both $s-d$ nodes. This cooperative transmission with the help of neighboring node(s) can increase throughput of the entire network and reliability of a packet delivery. The node which helps to relay the packet to the destination is referred to as a helper node. In this thesis, we focus on node cooperation frameworks on the link layer for VANETs to improve the system performance.

\subsubsection{Link-Layer Cooperation}

Traditionally, node cooperation for MAC protocols, also referred to as cooperative MAC protocols, in wireless networks have been proposed for faster transmission [42, 43], or to reduce the packet dropping rate [44]. Cooperation in the link-layer is done by the use of a suitable node which has good channel conditions to both the source and destination nodes. To exploit the benefits of node cooperation, several cooperative MAC protocols have been proposed for the legacy IEEE 802.11 networks with distributed control [42, 43, 45, 46, 44, 47, 48, 49]. Such node cooperation schemes force neighboring nodes to stop their transmissions during the cooperative transmission for an $s-d$ pair. Nodes in the vicinity of the helper along with the $s-d$ pair should back-off their transmissions until the ongoing transmission finishes. In addition, the interference area increases with the introduction of helpers, which further increases the probability of hidden and exposed node problems. 
In $[50,51,52]$, a cooperation scheme in TDMA MAC is presented for infrastructure based wireless networks. In such networks, communication links are established between a central controller (or access point) and mobile nodes. Cooperation is performed by dedicated (fixed) helper nodes and coordinated by the controller. In [51], dedicated time slots are allocated for helper nodes, even if cooperation is not required. Hence, these schemes cannot be applied directly in VANETs. Different from the existing works, when VANETs use D-TDMA, all operations such as cluster formation, slot allocation and cooperation decisions must be performed in a distributed manner. In $[53,54,55]$, node cooperation schemes with distributed cooperation decisions are presented. In [53], helper nodes perform dynamic cooperative retransmission to transmit packets to the target receiver during the source node's time slot. In the absence of helper nodes, the source node retransmits the same packet. Application of such cooperative retransmission to VANETs is not straightforward as each node with a time slot must broadcast its neighborhood information to its nearby nodes in every frame, in order to continue using its time slot in the next frame. In $[54,55]$, cooperative retransmission for multi-hop communication is achieved using idle time slots. It requires acknowledgement (ACK) from the target relay node, during the source node's time slot. Potential helper nodes participate in cooperation if they have the packet and do not receive ACK from the relay node. Such a scheme requires a large (and may be variable) time slot duration in order to accommodate ACKs during the source node's time slot, which is not desirable as it adds communication overhead. Hence the aforementioned node cooperation schemes cannot be applied directly in VANETs.

\subsubsection{Cooperation for Makeup Transmission}

A makeup strategy is a process to proactively correct the transmission failures that might have happened when a source node broadcasts a packet targeting to its one-hop neighbors, before the source node detects and/or corrects them [56]. Various strategies have been proposed for makeup transmissions to improve the reliability and effectiveness of broadcast service in wireless networks. In an opportunistic forwarding based makeup scheme [56], nodes (that received the packets from a source node) rebroadcast the packets with probability 1, until the predefined QoS is achieved. In a probabilistic method, neighboring nodes of a source node relay the packets with a predetermined probability [57]. However, these 
probabilistic relay schemes do not address the effects of dynamic networking conditions. Such effects include wastage of makeup opportunities when relay nodes are not in a good channel condition to the nodes that failed to receive the packet from the source node. On the other hand, a makeup strategy based on a weighted probability accounts for the distance or position from the source node to calculate the probability of retransmission [56, 58]. The makeup strategy is suitable for relaying a packet in a multi-hop scenario, so that

packets are forwarded to the nodes beyond one-hop transmission distance from the source node. Receive signal strength (RSS) based schemes use instantaneous channel condition information to perform makeup transmissions [59], at the cost of additional overhead in terms of signalling and time to choose the best relay node. The approach requires a longer, and may be a variable, time slot duration to accommodate the signalling and relay selection, which is not desirable in D-TDMA MAC. Thus, the existing makeup strategies either do not deal with the dynamic networking scenario in VANETs or is not suitable for D-TDMA MAC based broadcast services. Furthermore, we need to consider the node cardinality of relay nodes, such that a maximum number of nodes, that fail to receive the packet from the source node, will receive the packet before it expires.

\subsection{Motivation and Research Contributions}

Dynamic topology changes, high relative mobility among nodes, and frequent changes in vehicle density are some factors that impose challenges in VANETs, making the development of a MAC protocol extremely difficult. D-TDMA MAC, such as ADHOC MAC [35] and VeMAC [40], provide a collision-free broadcast service in VANETs with acknowledgement (ACK) from all the receivers within one-hop transmission distance. In the infrastructure based TDMA MAC, such as in cellular networks, the central controller proactively coordinates the nodes to avoid transmission collisions, where a node is scheduled to transmit in a time slot mainly when it has a packet to transmit. In contrast, in D-TDMA MAC due to the absence of a central controller, each node requires to exchange control signals for transmission scheduling, which is not efficient. To avoid spending a large amount of transmission opportunities in exchanging communication overheads, the number of time slots in each frame and the duration of each time slot are considered the system parameters 
and kept fixed in D-TDMA MAC. On the other hand, variation in vehicle density in the space and time results in under-utilization of the radio resources, in terms of unused time slots in a frame. The under-utilization occurs when there are not enough nodes in a two-hop neighborhood to use all the time slots of a frame. Moreover, D-TDMA MAC does not have any makeup strategy to handle a transmission failure resulting from the wireless channel impairments in VANETs. As frequent link breakage due to the dynamic networking conditions is common in VANETs, the lack of a makeup strategy in D-TDMA poses technical challenges in satisfying the strict QoS requirements. Hence, D-TDMA MAC is not always capable to support the wide rage of mobile distributed applications, specifically when the channel is in a poor condition and results in the wastage of time slots. Furthermore, it may not always be reliable to support delay sensitive messages for high priority safety applications. Though node cooperation can be use to alleviate such problems, through cooperative relay transmissions and/or makeup transmissions, the existing works in link-layer cooperation cannot be applied directly in VANETs (as discussed in the previous section). Hence, VANETs require new solutions in its MAC layer as well as in cooperative communication to address the associated issues. A cooperation scheme in the MAC layer should be designed to support the wide range of applications and must provide efficient and reliable communication with fast broadcast service to satisfy strict QoS requirements.

In this thesis, we focus on developing a node cooperation framework, as one possible way to overcome the existing challenges, in the link-layer for reliable communication in VANETs. The framework is expected to enable cooperation among nodes when an ongoing transmission fails, and prevent possible packet dropping. In addition, the proposed framework can help to facilitate safety related applications to quickly broadcast the corresponding packets in an area of interest. In developing such a node cooperation framework, this thesis has the following research contributions:

- A node cooperation framework is developed for point-to-point communication in D-TDMA based MAC protocols for VANETs, which enables an efficient utilization of the available radio resources, improves the network throughput, and reduces the packet dropping rate $[60,61]$;

- In the presence of high relative node mobility and channel fading, the effects of dynamic networking condition on the node cooperation framework are studied and a 
collision avoidance scheme is proposed to utilize cooperation opportunities efficiently without disrupting the normal operations of VANETs $[62,63]$;

- A node cooperation based makeup transmission framework is developed to guarantee the dissemination of high priority safety packets quickly to the maximum number of vehicles in an area of interest, without compromising the reliability [64].

\subsection{Organization of This Thesis}

The rest of this thesis is organized as follows. Chapter 2 describes our system model and assumptions. In Chapter 3, we present a novel cooperative MAC (CAH-MAC) protocol customized for D-TDMA MAC in VANETs. Performance evaluation of CAH-MAC is conducted to investigate its efficiency and reliability and compared with existing approaches $[61,60]$. Chapter 4 presents the effects of node cooperation on the operations of D-TDMA MAC. Enhanced CAH-MAC (eCAH-MAC), with collision avoidance and other additional features, is proposed to tackle the negative effects of node cooperation. Through extensive simulations and mathematical analysis, performance evaluation of eCAH-MAC is conducted and compared with existing approaches $[62,63]$. Chapter 5 presents the node cooperation for broadcast service in D-TDMA MAC. A node cooperation based makeup strategy is proposed with several helper selection schemes. The ability of the proposed framework to support safety applications in VANETs is investigated with optimization problem formulation and simulations [64]. Finally, Chapter 6 concludes this thesis and outlines some research topics for further investigation. 


\section{Chapter 2}

\section{System Model}

This chapter describes the system model under consideration and necessary assumptions made regarding network topology, communication among nodes, channel access, and node distribution.

\subsection{Network Topology and Communication Among Nodes}

Consider a VANET consisting of vehicles moving along a one-way multi-lane road segment. Vehicles are distributed randomly along the road and moving with the same average speed. Let $L$ be the number of lanes, each with width $w_{l}, l \in\{1,2,3, \ldots, L\}$. Vehicles separated by more than the distance $R$, referred to as transmission range, cannot communicate with each other, taking account of a possible poor channel condition. A node transmits its packet with a constant and single level transmission power, denoted as $P_{t}$. Let $\gamma_{r}$ denotes the instantaneous received power at a receiving node which is at $r$ meters from a transmitting node. In order to successfully decode a packet from a transmitting node, the instantaneous received power $\gamma_{r}$ must be equal to or greater than a threshold value, denoted as $\gamma_{t h}$. 


\subsection{Channel Access}

We focus on the control channel, which is used to broadcast safety messages and control information among one-hop neighbors and for negotiation between a pair of service provider and user through point-to-point communication (see Appendix A for the detail of multichannel operations in VANETs). The channel access mechanism is based on distributed TDMA MAC protocols, such as ADHOC MAC and VeMAC, such that the channel time is partitioned into frames and each frame is further partitioned into time slots. Each time slot is of a constant time interval and each frame consists of a fixed number of time slots, denoted by $F$. Each vehicle knows the number of time slots per frame and is capable of detecting the start time of a frame and, consequently, the start time of a time slot.

\subsection{Time Synchronization Among Vehicles}

Partitioning the channel time into time slots and accessing a time slot demand precise time synchronization among nodes. When a vehicle is equipped with a Global Positioning System (GPS) receiver, the one-pulse-per-second (1PPS) signal [65, 66] that a GPS receiver gets every second can be used for the time synchronization. The rising edge of each 1PPS signal can be taken as the reference instance of the start of a frame as illustrated in Fig. 2.1. The number of frames per second is an integer, denoted by $T$ (in frame per second). With the knowledge of the number of time slots per frame, the number of frames per second, and the duration of a time slot, each vehicle can detect the start time of a frame.

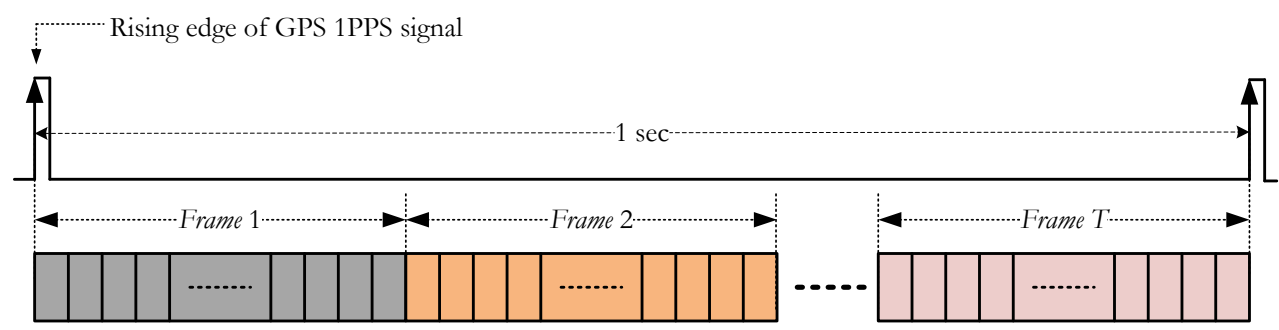

Figure 2.1: Use of the 1PPS signal for time synchronization. 
If the GPS signal is lost, a GPS receiver's local oscillator can be used for a short duration and a distributed synchronization scheme can be used for a longer duration, to synchronize nodes [67]. Details of such synchronization schemes are out of the scope of this thesis.

\section{$2.4 \quad$ Neighboring Nodes}

Each vehicle maintains lists of its one-hop and two-hop neighbors, based on information exchanged among nodes within transmission range $R$. One-hop and two-hop nodes are those which can be reached at maximum one and two hops of transmission respectively from a reference node. Sets of these nodes are called one-hop set (OHS) and two-hop set (THS) respectively. Furthermore, a node can communicate directly with any nodes in its OHSs. Similarly, all nodes in the same THS can communicate with each other with maximum two hops. Fig. 2.2 illustrates one-hop and two-hop sets with node $A$ as a reference. The reference node $A$ is a member of two OHSs namely OHS 1 and OHS 2 and a THS namely THS 1.

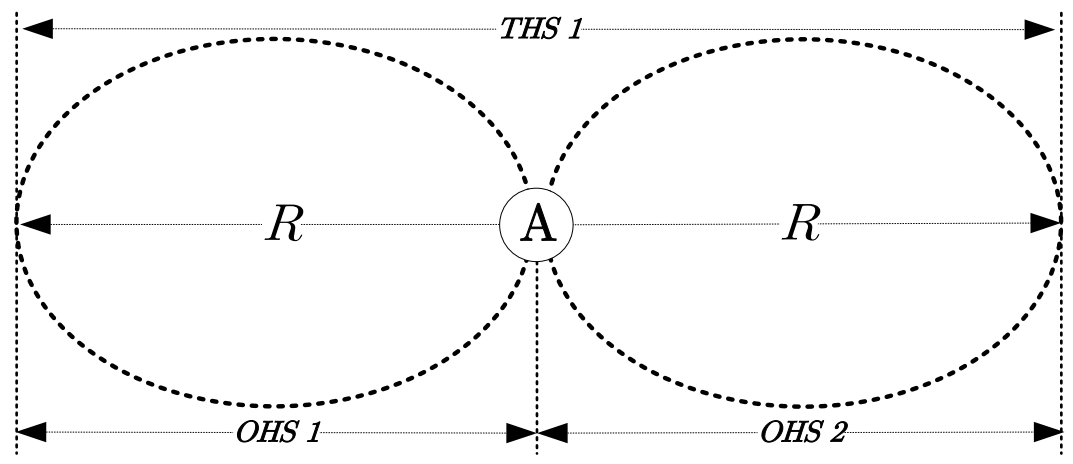

Figure 2.2: Illustration of a two-hop set with node $A$ as a reference, where an ellipse represents an OHS such that all nodes inside one ellipse can directly communicate with each other.

Nodes form clusters of THS neighbors. Here a cluster refers to a group of nodes which are at a maximum two-hop transmission distance from each other. There is no cluster head, and a node can be a member of multiple clusters. Formation of a THS can help to stop 
simultaneous usage of a time slot by more than one node within the same interference range and thus avoids the hidden node problem.

\subsection{Reservation and Retention of Time Slots}

In each time frame, a node transmits in its own time slot, if it already has a reserved time slot, or selects a time slot to perform time slot reservation based on its neighborhood and time slot usage information, otherwise. Nodes belonging to the same THS contend with each other to reserve a time slot. To reserve a time slot, a node first listens to the channel over the period of $F$ consecutive time slots (not necessarily in the same frame), then attempts to reserve one time slot among the unreserved ones if available. Access collisions occur when more than one nodes within the same interference range attempt to reserve the same time slot. After successfully reserving a time slot, a node transmits a packet in its own time slot in every frame until it encounters a merging collision [35] due to relative mobility. A merging collision occurs when nodes using the same time slot but belonging to different clusters approach each other, resulting in a transmission collision in the corresponding time slot [39]. In [39], it is shown that ADHOC MAC suffers from merging collisions due to node mobility resulting in throughput reduction. To overcome the throughput reduction, VeMAC is proposed in [40]. In VeMAC, time slots are separated into three disjoint groups, dedicated to vehicles moving in opposite directions and to RSUs respectively. Separation of the time slots into three disjoint groups alleviates throughput reduction by minimizing merging collisions due to relative node mobility.

Here, with a focus on cooperation to improve system throughput and transmission reliability, we consider a network where all nodes are perfectly synchronized in time. All operations such as reserving a time slot, time-synchronization among nodes, packet transmission are done in a distributed manner, making them suitable for VANETs [40, 41]. All vehicles have the same $R, P_{t}$ and $\gamma_{t h}$ values. 


\section{Chapter 3}

\section{Cooperation in ADHOC MAC}

In this chapter, we present a node cooperation scheme for VANETs mainly focusing on the MAC layer, called Cooperative ADHOC MAC ${ }^{1}$ (CAH-MAC) [60, 61]. As discussed in Section 1.3.1, existing works on link-layer cooperation are not suitable for VANETs scenarios and cannot be applied directly. Different from the existing works, here we consider a VANET using a distributed TDMA based MAC. In the system, nodes reserve their time slots and nearby nodes form a cluster to share a time frame. For cooperation at the link layer, a helper node utilizes an idle time slot to relay a packet that failed to reach the destination in a direct transmission due to a poor channel condition, without affecting the normal (non relay) transmissions. Using idle time slots for the cooperative relay transmissions, the proposed CAH-MAC protocol improves system throughput and transmission reliability of the VANET.

With a focus on cooperation to improve transmission efficiency, we consider a network where vehicles have already reserved their time slots and have information to transmit targeting specific destination nodes in each frame. Thus, vehicles access the channel once in every frame in their respective time slots. Access collisions do not occur and cooperation is performed by only those nodes which have their own slots for transmission. Furthermore, vehicles move with negligible relative mobility over an observation period (duration of a

\footnotetext{
${ }^{1}$ The name Cooperative ADHOC MAC is given as node cooperation is performed over the distributed TDMA MAC namely ADHOC MAC.
} 
time frame). Hence, they are stationary with respect to each other, maintaining a fixed network topology during the observation period. As the relative mobility among nodes is negligible, merging collisions do not occur; hence a reserved time slot is always dedicated to its owner. To investigate the effects of channel quality, we consider that the vehicles within the transmission range of a source node can successfully receive the transmitted packets with probability $p$, taking account of a possible poor channel condition. The probability $p$ depends on channel characteristics. The smaller the $p$ value, the poorer the channel quality. The parameter $p$ does not account for transmission errors due to collisions when multiple nodes within an interference range transmit simultaneously.

We derive a close-form expressions for the throughput and packet dropping rate of the newly proposed CAH-MAC protocol, which is verified using simulations. Our analysis shows that the $\mathrm{CAH}-\mathrm{MAC}$ protocol achieves a higher throughput and a lower packet dropping rate than that of the ADHOC MAC under a similar networking condition. Numerical results demonstrate that performance gain by cooperation is significant for a moderate channel condition. In addition, the gain is significant in the presence of a moderate number of nodes in a two-hop neighborhood relative to the number of time slots available in a frame.

\subsection{Protocol Description}

In this section, we discuss the detail operation of CAH-MAC, including cooperation decision and helper selection. A node in its own time slot transmits a packet that consists of frame information $(\mathrm{FI})$, cooperation header $(\mathrm{COH})$, packet header $(\mathrm{PH})$, payload data, and cyclic redundancy check (CRC). Fig. 3.1 shows the structure of a packet that a node transmits. The packet header, payload data, and $\mathrm{CRC}$ are the same as in ADHOC MAC and VeMAC, whereas frame information is different. In addition, $\mathrm{COH}$ is a new field that is introduced specifically for cooperation in CAH-MAC. In the following, we describe the structure and purposes of the signalling fields, namely the frame information and $\mathrm{COH}$. 


\begin{tabular}{|c|c|c|c|c|}
\hline $\begin{array}{c}\text { Frame } \\
\text { Information } \\
(\mathrm{FI})\end{array}$ & $\begin{array}{c}\text { Cooperation } \\
\text { Header } \\
(\mathrm{COH})\end{array}$ & $\begin{array}{c}\text { Packet } \\
\text { Header } \\
(\mathrm{PH})\end{array}$ & Payload Data & CRC \\
\hline
\end{tabular}

Figure 3.1: Packet structure in CAH-MAC.

\subsubsection{Frame Information}

The fame information (FI) is a collection of ID fields (IDFs). The number of IDFs in an FI field is equal to $F$, i.e., the number of time slots per frame. Each IDF is dedicated to the corresponding time slot of a frame. The basic FI field structure is shown in Fig. 3.2. Temporary (or short) identifier with the size of ( $1-2$ bytes) [35, 40], which is shorter than the size of a MAC address, can be used as an ID of a node. Such a short ID can be selected randomly by a node and changed if there is a conflict [35]. Use of such a short ID reduces the size of the FI in a packet and, hence, reduces the MAC overhead.

\begin{tabular}{|c|c|c|c|c|c|}
\hline IDF-1 & IDF-2 & IDF-3 & --- & IDF-(F-1) & IDF-F \\
\hline$i d_{a}$ & $\phi$ & $i d_{b}$ & --- & $\phi$ & $i d_{z}$ \\
\hline
\end{tabular}

Figure 3.2: Structure of a frame information field, where $\phi$ indicates an empty field.

Destination node $D$, upon receiving a packet successfully from source node $S$ in the $s^{\text {th }}$ time slot, concludes that the $s^{\text {th }}$ time slot belongs to node $S$. Node $D$ then puts the ID of node $S$ in the $s^{\text {th }}$ IDF of its FI. By successfully receiving a packet from node $S$, node $D$ knows $(i)$ the existence of node $S$ as its one-hop neighbor, (ii) node $S$ is the owner of the $s^{\text {th }}$ time slot, and finally ( $\left.i i i\right)$ all the one-hop neighbors of node $S$ and their corresponding time slots. Hence, by successfully receiving FIs from all of its one-hop neighbors, a node maintains a neighbor-table which includes: $(i)$ all of its one-hop neighbors, $(i i)$ all of its two-hop neighbors, and (iii) the owner of each time slot in a frame. If there is no signal in a time slot, then a node treats it as an unreserved time slot. In such a case, corresponding IDFs of unreserved time slots are left empty in an FI field as illustrated in Fig. 3.2 for 
IDF-2.

A node can identify an unreserved time slot in which it can transmit without causing any collision in its one-hop neighborhood. Note that a node updates its neighbor-table based on any packets received successfully from new neighbors. These packets can be broadcast, unicast, or multicast packets. In addition to the neighborhood discovery, formation of a THS cluster, and time slot reservation, the FI helps for transmission acknowledgement. For example, consider that node $D$ does not include the ID of node $S$ in the IDF- $S$ of its FI. Upon receiving the FI from node $D$, node $S$ concludes a transmission failure between itself and node $D$ in the $s^{\text {th }}$ time slot, which is basically a negative acknowledgement (NACK). Similarly, inclusion of the node $S$ ID in the FI of node $D$ serves as acknowledgement (ACK) of a successful transmission from node $S$ to node $D$.

\subsubsection{Cooperation Among Neighboring Nodes}

Cooperation is always performed through a one-hop neighbor of the source and destination nodes. Since the channel condition may remain the same during the unused time slot as that of during the source node's time slot, retransmission by the source node during the unused time slot is not likely to be helpful and will waste the transmission opportunity. On the other hand, cooperative relay transmission of a packet, through an independent channel (i.e., between the helper and destination) during an unreserved time slot provides transmission diversity and, hence, improves transmission reliability even if the channel condition between an $s-d$ pair is poor [68]. In the following, we discuss how a node decides and performs cooperation. Let $\mathcal{F}=\{1,2,3, \ldots, F\}$ be the set of time slots in a frame. Let $\mathcal{O}_{x}$ and $\mathcal{T}_{x}$ be the OHS and THS of a node $x$. Let $\mathcal{B}_{x}$ be the set of all time slots which belongs to the THS of node $x$, i.e., any time slot $t \in \mathcal{B}_{x}$ is reserved for node $x$. Consider $S$ and $D$ as the source and destination nodes with the $s^{\text {th }}$ and $d^{\text {th }}$ time slots respectively and node $H$ as the helper node. Cooperation decision and cooperative relay transmission are performed only if all the following conditions are satisfied:

1. The direct transmission fails: Cooperation is trigged when the direct transmission between the source and the destination fails. Upon a transmission failure, node $D$ does not acknowledge the transmission from node $S$, such that $S \notin \mathcal{O}_{D}$. Potential 
helper nodes have the transmission failure information after receiving the FI from node $D$.

2. The helper successfully receives a packet for retransmission: A node can potentially offer cooperation only if it receives the packet successfully from the source node $S$ during the $s^{\text {th }}$ time slot.

3. The destination is reachable: Node $H$ can relay a packet that node $D$ failed to receive from node $S$, if node $D$ is within its transmission range. Hence both source node $S$ and destination node $D$ must be listed as one-hop neighbors in node $H$ 's neighbor-table, i.e., $S, D \in \mathcal{O}_{H}$.

4. There is an available time slot: Helper node $H$, when conditions 1) - 3) are satisfied, can offer and perform cooperation if there exists at least one unreserved time slot $h \in \mathcal{F}$ during which it can transmit. The transmission from $H$ in time slot $h$ shall not cause any collision at its two-hop neighbors, i.e. $\forall h \notin \mathcal{B}_{H}$.

If all the preceding conditions are satisfied, helper node $H$ offers cooperation to the source and destination and the cooperative transmission is performed in time slot $h$. If there are multiple potential helper nodes, the one which first announces to help will relay the packet while all other potential helpers will not proceed with cooperation for the same packet. As the potential helper nodes wait for their respective time slots to offer cooperation, for a given $s-d$ pair, helper node $H$ among all the potential helper nodes is the one whose time slot is the earliest one after the detection of transmission failure. Fig. 3.3 shows necessary information exchanges for cooperation in the CAH-MAC. When destination node $D$ fails to receive a packet from source node $S$ (in Fig. 3.3(a)), it announces transmission failure through its FI as shown in Fig. 3.3(b). Upon deciding to cooperate, helper node $H$ transmits its intention of cooperation using cooperation header $(\mathrm{COH})$ as in Fig. 3.3(c). Note that as helper node $H$ 's time slot is earlier than that of potential helper node $H_{1}$, node $H$ offers cooperation and node $H_{1}$ suspends its cooperation decision after receiving $\mathrm{COH}$ from node $H$. In the $h^{\text {th }}$ time slot, after receiving a cooperation acknowledgement (C-ACK) from the destination node $D$, helper node $H$ transmits the packet that node $D$ failed to receive (in Fig. 3.3(d)). Next, we discuss $\mathrm{COH}$ that a helper node uses to offer 
cooperation and $\mathrm{C}$-ACK that a destination node uses to avoid collision during cooperative relay transmission.

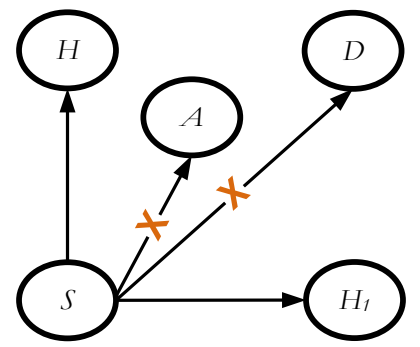

(a)

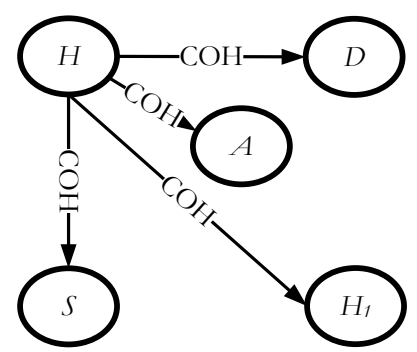

(c)

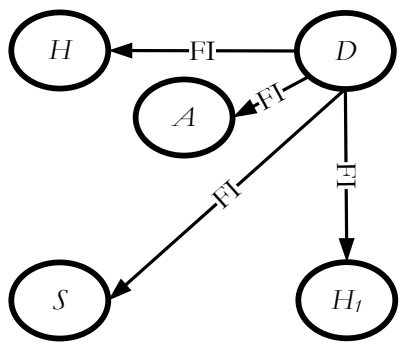

(b)

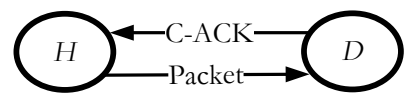

(d)

Figure 3.3: Information exchanges in the CAH-MAC: (a) Phase 1: Source node fails to transmit a packet to the destination; (b) Phase 2: Neighboring nodes detect transmission failure after examining the FI from the destination; (c) Phase 3: Helper node $H$ offers cooperation; (d) Phase 4: Helper node $H$ re-transmits the packet that failed to reach the destination after receiving a cooperation acknowledgement from the destination.

\subsubsection{Cooperation Header and Cooperation Acknowledgement}

Once a node decides to cooperate, it transmits its decision via $\mathrm{COH}$ in its packet. The following information is included in the $\mathrm{COH}$ : $(i)$ its intention to cooperate, $(i i)$ the index of time slot of the source during which transmission failure occurred, and (iii) the index of the selected unreserved time slot in which the packet will be retransmitted from the helper to the destination. The aforementioned information is embedded in the $\mathrm{COH}$ and transmitted in the helper's time slot. Other potential helpers (which can offer cooperation 


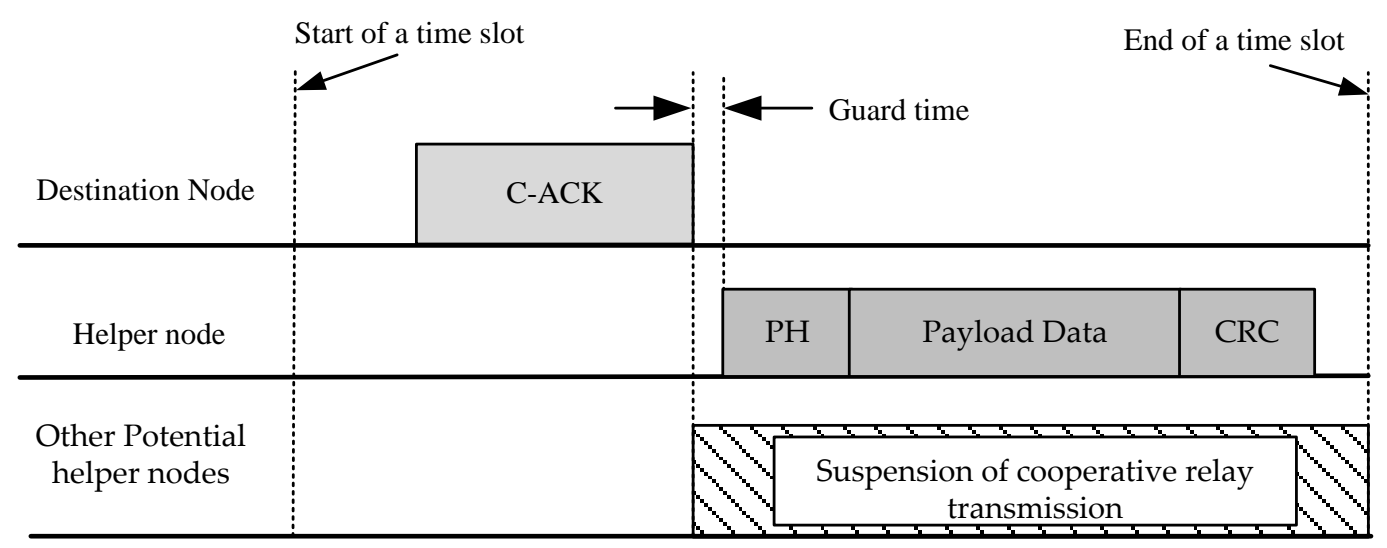

Figure 3.4: Cooperative relay transmission during an unreserved time slot.

and are in the OHS of helper node) suspend their intentions, once they receive cooperation decision from helper $H$. Hence, helper node $H$ is the one which first offers cooperation and performs cooperation for the $s-d$ pair. Such a suspension of cooperation intention from other potential helpers avoids collision during cooperative relay transmission. However, collisions may occur at the destination node when two or more potential helpers, which are not in each other's OHS, offer cooperation at the same unreserved time slot. In order to avoid such collisions, C-ACK from the destination node is transmitted during the selected unreserved time slot, which is illustrated in Fig. 3.4. In C-ACK, the destination node puts the ID of the first potential helper which offered cooperation to accept cooperation. Transmission of the C-ACK signal from the destination node forces other potential helpers to suspend their transmissions, thus avoiding any possible collision. After receiving C-ACK from the destination node, helper node $H$ retransmits the packet that failed to reach the destination in the direct transmission from the source node.

The size of a short ID is always enough to be shared among the nodes that are sharing a frame (at a time a maximum number of $F$ nodes can share a frame). Hence, the size of an index of a time slot is comparable with the size of a short ID. Consequently, the size of a $\mathrm{COH}$ is negligible as compared to the size of FI (and obviously the size a time slot), which has a space for $F$ IDs. Generally, the $F$ value is set large enough to guarantee a time slot for each node. In addition, C-ACK and cooperative transmission are performed 
in an unreserved time slot. Hence, cooperation can be performed at the cost of negligible overhead as compared to a time slot which would be wasted in the absence of cooperation. It is to be noted that, in the proposed CAH-MAC, only one helper performs the cooperative relay transmission for a failed $s-d$ direct transmission. Potential helpers, which can offer cooperation to the failed $s-d$ direct transmission, suspend their cooperation intentions once they receive cooperation decision from the helper node. Hence, a potential helper offers cooperation to only those failed $s-d$ direct transmissions which are not offered with cooperation, but not to every failed $s-d$ direct transmissions. This reduces the size of $\mathrm{COH}$ and hence the communication overhead due to cooperation.

\subsection{Preliminaries for the Performance Analysis}

In this section, we present preliminaries required for the performance evaluation of the proposed CAH-MAC protocol. Throughput and reliability are taken as metrics to compare the performance of CAH-MAC with ADHOC MAC.

\subsubsection{Node Distribution}

Vehicles are distributed randomly on the road with an exponentially distributed intervehicular distance over each lane. The assumption is valid when the roadways are not in their full capacity and vehicles have low interaction among each other, such that the intervehicle distances are independent and identically distributed (i.i.d.) with an exponential probability density function (pdf) [69]. Let $\rho_{l}, l \in\{1,2,3, \ldots, L\}$, be the vehicle density of lane $l$ in terms of the number of vehicles per unit length. Thus the counting of vehicles follows a Poisson process over a given length of road, such that the probability of finding $n$ vehicles along a given length $z$ of the road segment is given by

$$
p(n, z)=\frac{(\rho z)^{n} e^{-\rho z}}{n !}, \quad n=0,1,2, \ldots
$$

where $\rho=\sum_{l=1}^{L} \rho_{l}$. 


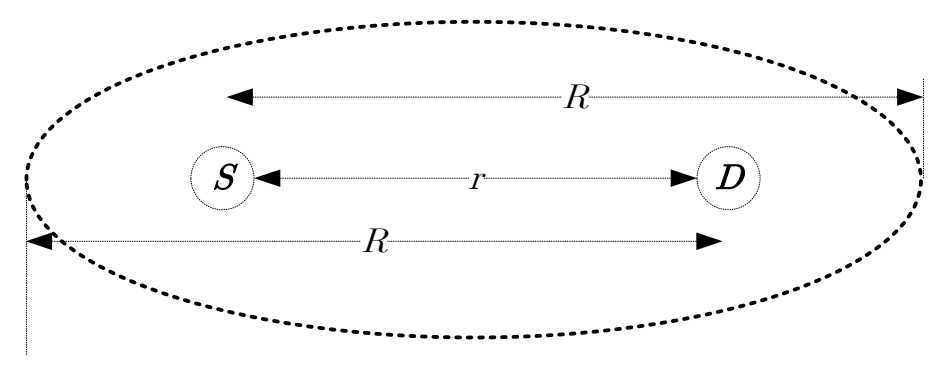

Figure 3.5: Illustration of the common coverage road segment of an $s-d$ pair.

Note that (3.1) is an approximation for tractable analytical framework, considering a vehicle as a point in a line representing a roadway. In reality, the inter-vehicular distance follows a shifted negative exponential distribution [70], such that a minimum safety distance (MSD) is always maintained by two adjacent vehicles in a lane to avoid any vehicle collision between them. In [61], it is shown, via computer simulation, that such an assumption is valid for the performance analysis.

\subsubsection{Distribution of the Neighboring Node Numbers}

Let $N_{T}$ be the number of nodes in a given THS, including all nodes within distance of $R$ meters both in backward and forward directions of the reference node (including itself). Fig. 2.2 illustrates that nodes in the given THS distributed along the road segment of length $2 R$ units. As the counting of nodes follows a Poisson distribution, the probability mass function (pmf) of $N_{T}$ can be obtained by substituting $z=2 R$ in (3.1), given by

$$
\operatorname{Pr}\left\{N_{T}=n_{t}\right\}=p\left(n_{t}, 2 R\right)=\frac{(2 \rho R)^{n_{t}} e^{-2 \rho R}}{n_{t} !}, \quad n_{t}=0,1,2, \ldots
$$

A node cannot access time slots that are being used by its THS members. Thus for stable performance, $F$ must be large enough so that each node located within a road segment of $2 R$ units gets a unique time slot. Hence, we should have $F>\bar{N}_{T}=2 \rho R$ to achieve stable performance of the MAC protocol.

Next, consider the number of nodes which are within the transmission range from both 
the source and the destination, denoted by $N_{o}$. Fig. 3.5 illustrates the road segment where such nodes are distributed. In Fig. 3.5, nodes which are in OHS of both source and destination are distributed along the road segment of length $2 R-r$ units, where $r(\leq R)$ is the distance between the source and destination nodes. If $r$ is uniformly distributed within $[0, R]$, the average distance between the source and destination is $0.5 R$. Consequently, common OHS nodes are distributed along length of $1.5 R$ units on average. The pmf of $N_{o}$ can be obtained by substituting $z=1.5 R$ in (3.1), given by

$$
\operatorname{Pr}\left\{N_{o}=n_{o}\right\}=p\left(n_{o}, 1.5 R\right)=\frac{(1.5 \rho R)^{n_{o}} e^{-1.5 \rho R}}{n_{o} !}, \quad n_{o}=0,1,2, \ldots
$$

\subsubsection{Transmission During a Time Slot}

A node performs direct transmission during its own time slot, i.e, during a reserved time slot, or cooperative relay transmission during an unreserved time slot. Within the transmission range $R$, the channel is characterized by $p$ such that the probability of successful communication in a given time slot is given by

$$
\operatorname{Pr}\left\{\gamma_{r} \geq \gamma_{t h}\right\}= \begin{cases}p, & \text { if } 0<r \leq R \\ 0, & \text { otherwise }\end{cases}
$$

where $r$ is the distance between an $s-d$ pair. Let $p_{s}$ denote the probability of successful transmission during a time slot. As channel condition and transmission collision are independent of each other, $p_{s}$ is given by

$$
p_{s}=\left(1-p_{c}\right) p
$$

where $p_{c}$ is the probability of transmission collisions in a given time slot. Collisions can be merging collisions [35] and are due to relative mobility between nodes. Since nodes are relatively stationary with respect to each other in the system model under consideration, there are no collisions among packets transmitted by different nodes. Hence $p_{c}=0$ and $p_{s}=p$. In the following, we use $p_{s}$ to derive necessary distribution functions required for the performance analysis of the CAH-MAC. 


\subsubsection{Types of Time Slots and Their Distributions}

In a given THS, out of the total $F$ time slots in a frame, each time slot is one of the following types:

- Unreserved: Time slots which are not reserved by any node are unreserved time slots. Any time slots other than unreserved in a frame are reserved time slots. Let random variable $U$ represents the number of unreserved time slots in a frame.

- Successful: The reserved time slots during which packets are successfully delivered to the target destinations are regarded as successful time slots. Let random variable $X$ represents the number of successful time slots in a frame. Hence, given $U=u$, we have $0 \leq X \leq F-u$.

- Failed: Time slots other than unreserved and successful time slots in a frame are failed time slots.

Note that in cooperation enabled transmission, for each failed direct transmission, an unreserved time slot is used for the cooperative relay transmission if conditions in subsection 3.1.2 are satisfied. With cooperation, the corresponding time slot is considered a successful time slot if a packet is successfully delivered to the destination through the helper, and a failed time slot otherwise.

In a frame, out of $F$ available time slots, only $N_{T}$ time slots are reserved by members of the corresponding THS. Hence we have

$$
U= \begin{cases}0, & \text { if } N_{T} \geq F \\ F-N_{T}, & \text { if } 1 \leq N_{T}<F .\end{cases}
$$

Note that in (3.6), for a time slot to be called as reserved (or unreserved) or for a frame to exist, there must be at least one node in the corresponding THS, i.e., $N_{T} \geq 1$. If a frame exists, unreserved time slots are leftover time slots after all members of the THS finished their reservations. Hence, if a frame exists, $0 \leq U \leq F-1$. On the other hand, if there are more than $F$ members in a THS, there will be no unreserved time slot in the corresponding 
frame, i.e., $U=0$. Hence, from (3.2) and (3.6), the pmf of $U$ is given by

$$
\operatorname{Pr}\{U=u\}= \begin{cases}1-\sum_{i=1}^{F-1} \frac{(2 \rho R)^{i} e^{-2 \rho R}}{i !}, & \text { for } u=0 \\ \frac{(2 \rho R)^{F-u} e^{-2 \rho R}}{(F-u) !}, & \text { for } 0<u \leq F-1\end{cases}
$$

\subsubsection{Cooperation Enabled Transmission}

If a transmission failure occurs, cooperation may be triggered. Based on the operation procedure discussed in Section 3.1, cooperation gets triggered if all of the following events occur:

- Event $1\left(E_{1}\right)$ : There is at least one potential helper. Potential helpers are those nodes which are in the same OHS of the source and destination nodes. In addition, potential helpers successfully received the packet, during the source nodes's time slot, that failed to reach the destination.

- Event $2\left(E_{2}\right)$ : There exists at least one unreserved time slot in which a potential helper node can transmit without causing any collision(s) in its OHS neighborhood.

Event $E_{1}$ depends on the channel conditions between the source node and common one-hop neighbors of both source and destination nodes. On the other hand, from (3.6) and (3.7), event $E_{2}$ depends on the number of THS members of the helper node for the

given $F$ value. Hence, events $E_{1}$ and $E_{2}$ are independent of each other. The probability of cooperation decision for each failed direct transmission, $p_{\text {coop }}$, is given by

$$
p_{\text {coop }}=\operatorname{Pr}\left\{E_{1}\right\} \operatorname{Pr}\left\{E_{2}\right\}
$$

In the following, we derive close-form expressions of the probabilities of events $E_{1}$ and $E_{2}$ occurrence respectively.

\section{Existence of Potential Helpers (Event 1)}

The number of common OHS neighbors of an $s-d$ pair, which receive a packet from the source, follows a binomial distribution. These common one-hop neighbors are potential 
helpers of the $s-d$ pair. Let random variable $Y$ represent the number of potential helpers for a given failed packet transmission. Given the number of common nodes in the $S$ and $D$ 's OHSs $N_{o}=n_{o}$, potential helper does not exist if $n_{o} \leq 2$, as $s-d$ pair cannot be the helper. If $3 \leq n_{o} \leq F$, up to $n_{o}-2$ nodes can act as a helper if they successfully receive the packet from the source. Finally if $n_{o}>F$, only $F-2$ nodes which have reserved a time slot in a frame can act as a helper. A node, which does not own a time slot cannot transmit its $\mathrm{COH}$, hence it cannot perform cooperation. Therefore, given $N_{o}=n_{o}$, the pmf of $Y$ is given by

$$
\operatorname{Pr}\left\{Y=y \mid N_{o}=n_{o}\right\}= \begin{cases}1, & \text { for } y=0 \text { if } n_{o} \leq 2 \\
\left(\begin{array}{c}
n_{o}-2 \\
y
\end{array}\right) p_{s}^{y}\left(1-p_{s}\right)^{n_{o}-y-2}, & \text { for } 0 \leq y \leq n_{o}-2 \text { if } 3 \leq n_{o} \leq F \\
\left(\begin{array}{c}
F-2 \\
y
\end{array}\right) p_{s}^{y}\left(1-p_{s}\right)^{F-y-2}, & \text { for } 0 \leq y \leq F-2 \text { if } n_{o}>F .\end{cases}
$$

Event 1 occurs when at least one common one-hop neighbor of the $s-d$ pair successfully receive the packet from the source, i.e., $Y>0$. Given $N_{o}=n_{o}$, the probability of Event 1 occurrences is

$$
\operatorname{Pr}\left\{E_{1} \mid N_{o}=n_{o}\right\}=1-\operatorname{Pr}\left\{Y=0 \mid N_{o}=n_{o}\right\}
$$

From (3.3), (3.9), and (3.10), the probability of Event 1 occurrences can be derived as

$$
\begin{aligned}
\operatorname{Pr}\left\{E_{1}\right\} & =\sum_{n_{o}=3}^{F}\left(1-\left(1-p_{s}\right)^{n_{o}-2}\right) \frac{(1.5 \rho R)^{n_{o}} e^{-1.5 \rho R}}{n_{o} !} \\
& +\left(1-\left(1-p_{s}\right)^{F-2}\right)\left(1-\sum_{n_{o}=0}^{F} \frac{(1.5 \rho R)^{n_{o}} e^{-1.5 \rho R}}{n_{o} !}\right) .
\end{aligned}
$$




\section{Existence of Unreserved Time Slots (Event 2)}

For nodes belonging to the same THS, an unreserved time slot for one node is unreserved for all of them. Hence, a potential helper can help an $s-d$ pair if there exists at least one unreserved time slot in the frame belonging to the corresponding THS. Event 2 occurs if there exists at least one unreserved time slot in the frame, which is being shared by the source, destination, and potential helper nodes. From (3.7), we have

$$
\operatorname{Pr}\left\{E_{2}\right\}=\operatorname{Pr}\{U>0\}=\sum_{u=1}^{F-1} \frac{(2 \rho R)^{u} e^{-2 \rho R}}{u !}
$$

From (3.8), (3.11), and (3.12), the probability of cooperation, $p_{\text {coop }}$, can be calculated.

\subsubsection{Benefit of Cooperation}

Note that just triggering a cooperation does not guarantee a successful retransmission. Cooperation is beneficial only if the transmission from the helper to the destination is successful. With the introduction of cooperation, transmission is successful either direct or cooperative relay transmission is successful. Hence the probability of a successful transmission with cooperation, $p_{s}^{c o o p}$, is given as

$$
p_{s}^{c o o p}=p_{s}+p_{s}\left(1-p_{s}\right) p_{c o o p}
$$

\subsection{Throughput Analysis}

In this section, we derive close-form expressions for throughput and throughput gain of the proposed CAH-MAC.

\subsubsection{Expected Number of Successful Time Slots}

One way to evaluate the MAC protocol is by its ability to handle channel errors. In TDMA based MAC, the number of successful time slots per frame is an indication of this ability. 
If a MAC protocol is more robust in reliable packet delivery in a relatively poor channel condition, it can achieve a higher number of successful time slots in a frame. A time slot is successful only if the transmitted packet does not collide with packets from other nodes in the THS and successfully reaches the target receiver. Given $U=u$, the number of successful time slots, $X$, follows a binomial distribution with parameters $\left(F-u, p_{s}\right)$ and its conditional pmf is given by

$$
\operatorname{Pr}\{X=x \mid U=u\}=\left(\begin{array}{c}
F-u \\
x
\end{array}\right) p_{s}^{x}\left(1-p_{s}\right)^{F-u-x}, \quad x=0,1,2, \ldots, F-u .
$$

Consequently, the expected value of $X$ given $U=u$ is

$$
E[X \mid U=u]=(F-u) p_{s}
$$

From (3.7) and (3.15), the expected number of successful time slots, $E[X]$, can be written as

$$
E[X]=p_{s} \sum_{u=1}^{F-1}(F-u) \frac{(2 \rho R)^{F-u} e^{-2 \rho R}}{(F-u) !}+p_{s} F\left(1-\sum_{u=1}^{F-1} \frac{(2 \rho R)^{u} e^{-2 \rho R}}{u !}\right)
$$

Similarly, with the cooperation, the expected number of successful time slots in a frame as in (3.16) changes to

$$
E\left[X_{\text {coop }}\right]=p_{s}^{\text {coop }} \sum_{u=1}^{F-1}(F-u) \frac{(2 \rho R)^{F-u} e^{-2 \rho R}}{(F-u) !}+p_{s}^{\text {coop }} F\left(1-\sum_{u=1}^{F-1} \frac{(2 \rho R)^{u} e^{-2 \rho R}}{u !}\right)
$$

where $X_{\text {coop }}$ is a random variable representing the number of successful time slots in a frame with cooperation enabled transmissions.

\subsubsection{Throughput and Throughput Gain}

Throughput is defined as the fraction of successful time slots over the total number of time slots per frame, $F$. Let $\sigma$ and $\sigma_{\text {coop }}$ denote the throughput of ADHOC MAC and 
CAH-MAC respectively. We have

$$
\sigma=\frac{E[X]}{F}, \quad \sigma_{\text {coop }}=\frac{E\left[X_{\text {coop }}\right]}{F} .
$$

The normalized throughput gain achieved by cooperation is given by

$$
\sigma_{\text {gain }}=\frac{\sigma_{\text {coop }}-\sigma}{\sigma} .
$$

\subsection{Reliability Analysis}

In this section, we develop a mathematical model to study the reliability of the CAH-MAC protocol in terms of packet transmission delay and packet dropping rate.

\subsubsection{Packet Transmission Delay}

The packet transmission delay is defined as the number of frames that is required to successfully transmit a packet to the destination. Upon transmission failure, a source attempts retransmission of a packet until it successfully reaches the destination. Since in the system under consideration, the probability of successful transmission during a time slot depends only on channel characteristics, the number of retransmission attempts is independent of collision probability and follows a geometric distribution [71, 72]. Let random variables $M$ and $M_{\text {coop }}$ represent the packet transmission delay of ADHOC MAC and CAH-MAC respectively. Hence, the pmf of $M$ with parameter $p_{s}$, is given by

$$
\operatorname{Pr}\{M=i\}=\left(1-p_{s}\right)^{i-1} p_{s}, \quad i=1,2,3, \ldots
$$

Similarly, the pmf of $M_{\text {coop }}$ with parameter $p_{s}^{\text {coop }}$ is given by

$$
\operatorname{Pr}\left\{M_{\text {coop }}=i\right\}=\left(1-p_{s}^{\text {coop }}\right)^{i-1} p_{s}^{\text {coop }}, \quad i=1,2,3, \ldots
$$


Consequently, the expected values of $M$ and $M_{\text {coop }}$ are given by

$$
E[M]=\frac{1}{p_{s}}, \quad E\left[M_{\text {coop }}\right]=\frac{1}{p_{s}^{c o o p}}
$$

respectively.

\subsubsection{Packet Dropping Rate}

In a communication system, a packet is dropped by a source node from its buffer memory, when it fails to deliver the packet to the destination within the predefined time limit. In the system, we consider the time limit in terms of the number of frames. Let $M_{\text {max }}$ denote the maximum number of frames that a source node attempts to transmits a packet, referred as the maximum transmission limit. Hence, for a given $M_{\max }$ value, the packet dropping rate of $\mathrm{ADHOC} \mathrm{MAC}$, denoted as $P D R$, is given by

$$
P D R=1-\sum_{i=1}^{M_{\max }}\left(1-p_{s}\right)^{i-1} p_{s} .
$$

With the cooperation enabled transmission, packet dropping rate is given by

$$
P D R_{\text {coop }}=1-\sum_{i=1}^{M_{\max }}\left(1-p_{s}^{c o o p}\right)^{i-1} p_{s}^{c o o p}
$$

where $P D R_{\text {coop }}$ denotes the packet dropping rate of CAH-MAC.

In the next section, we present numerical results to validate the throughput and reliability analysis, and to evaluate performance gain by cooperation. 


\subsection{Numerical Results}

Simulations are performed in MATLAB. Five hundreds nodes are distributed along a road segment following the Poisson distribution ${ }^{2}$. Vehicle densities, $\rho_{l}$ (vehicle per kilometer), are kept equal in both lanes, hence $\rho=L \rho_{l}$. Each simulation result is obtained by simulating 200, 000 frames from 40 different network topologies. The value of $p$ is varied to characterize different channel conditions. Throughput, throughput gain, packet transmission delay and packet dropping rate of $\mathrm{CAH}-\mathrm{MAC}$ are obtained in comparison with ADHOC MAC for several different scenarios.

Figs. 3.6-3.8 compare the throughput of CAH-MAC with that of ADHOC MAC. It is observed that with an introduction of cooperation, throughput of CAH-MAC is in general higher than that of ADHOC MAC. However at two extreme channel conditions, i.e., $p=0$ and 1 , both protocols perform equally as expected. When $p=0$, all transmissions fail due to channel errors; thus there are no potential helpers. On the other hand, at $p=1$, all packets reach to the destination directly from the source; thus cooperation is not needed. The advantage of cooperation starts as $p$ increases from zero, such that a source node can get potential helpers upon a transmission failure. Fig. 3.9 shows the throughput gain of CAH-MAC over ADHOC MAC. Note that, at $p=0$ and $p=1$, the throughput of both protocols are equal, resulting in no throughput gain. All the simulation results match well with the analytical results.

Fig. 3.6 shows that the throughputs are proportional to the vehicle density per lane $\left(\rho_{l}\right)$ values. The smaller the density value, the smaller number of THS members. This tends to increase the number of unreserved time slots in a frame, which has a negative effect on the throughput. In ADHOC MAC, unreserved time slots are left unused, while in $\mathrm{CAH}-\mathrm{MAC}$ the introduction of a cooperation reduces transmission failure by utilizing unreserved time slots. Fig. 3.7 shows that the throughput increases with an increase in the transmission range $R$. For the given vehicle density, increasing the transmission range also increases the number of THS members, leading to more efficient utilization of available time slots in each frame. Fig. 3.8 shows the effect of the frame size $F$ on the throughput.

${ }^{2} \mathrm{~A}$ road segment with two lanes is considered. A line represents a lane that is 5 meters wide. Vehicles are represented by points on the lines. Hence, we have $L=2$ and $w_{l}=5 \mathrm{~m}$. 


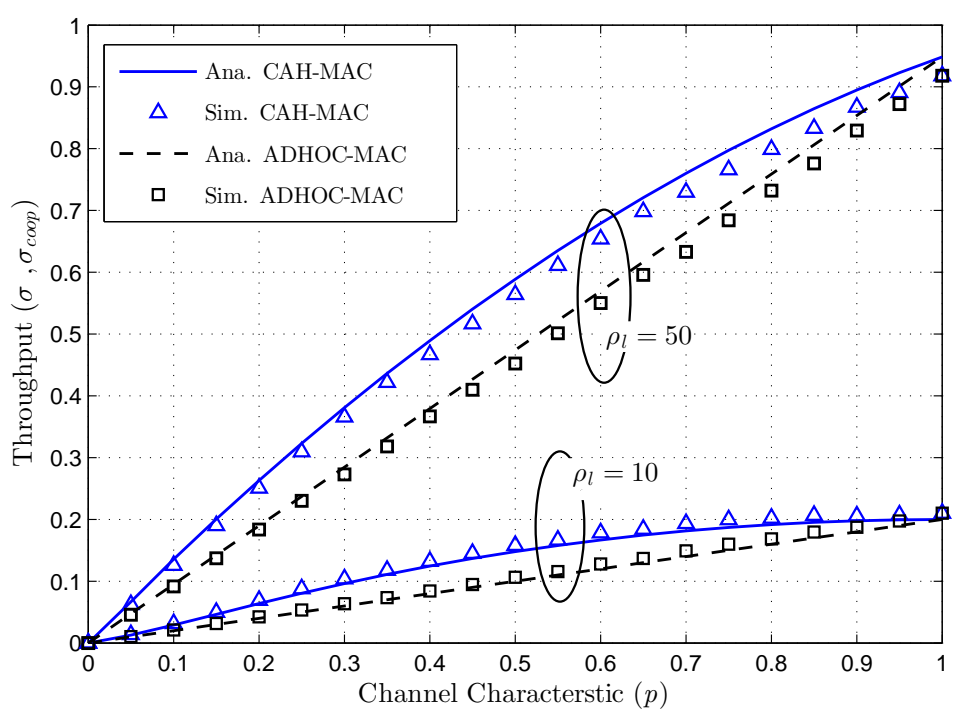

Figure 3.6: Throughput comparison of ADHOC MAC and CAH-MAC with $R=300 \mathrm{~m}$ and $F=60$ time slots ( $\rho_{l}$ is in vehicle per kilometer).

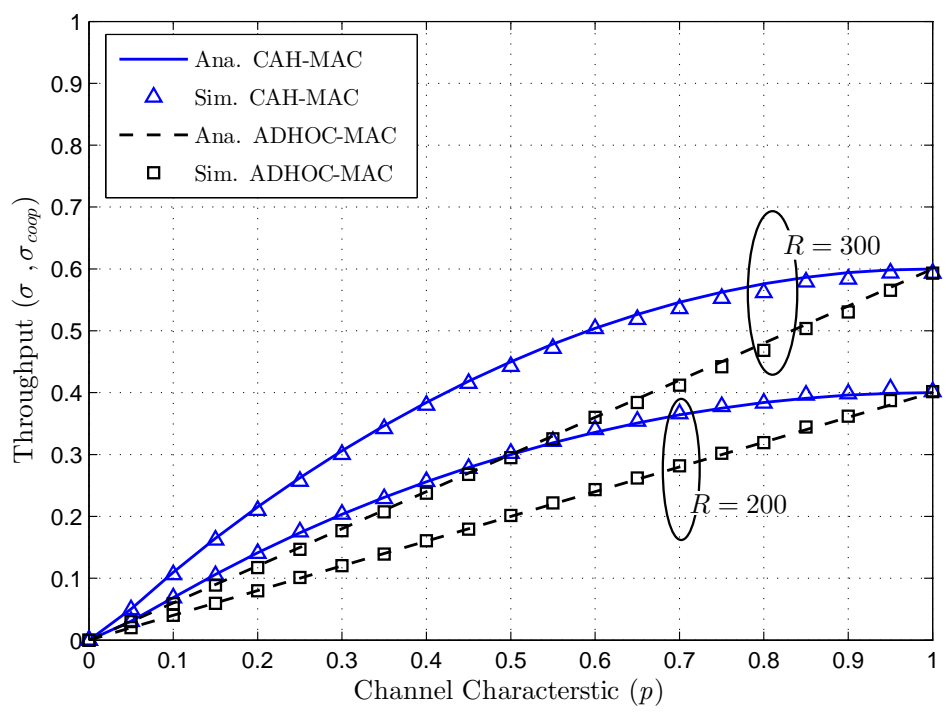

Figure 3.7: Throughput comparison of ADHOC MAC and CAH-MAC with $\rho_{l}=30$ vehicle per kilometer and $F=60$ time slots. 


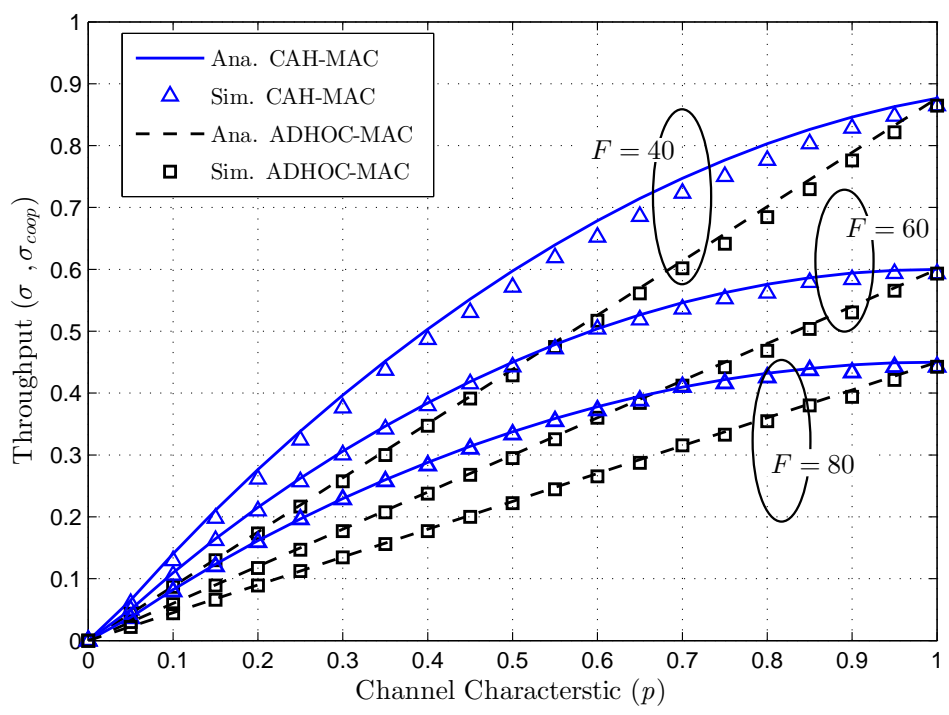

Figure 3.8: Throughput comparison of ADHOC MAC and CAH-MAC with $\rho_{l}=30$ vehicle per kilometer and $R=300 \mathrm{~m}$.

For the given vehicle density and transmission range, the throughput decreases with an increase in $F$. For the relatively small average number of THS members, an increase in $F$ increases the number of unreserved time slots and hence decreases the throughput. Figs. 3.6-3.8 show the increase in throughput of CAH-MAC over ADHOC MAC for the same $p$ value under a similar networking condition. In general, the throughput improvement by cooperation increases as the channel quality improves from a very poor condition (i.e., small $p$ value). The better channel quality increases the probability of successful relay transmission. On the other hand, as the channel quality further improves, the probability of successful direct transmission increases reducing the needs for cooperation and leading to a smaller cooperation gain in the throughput.

Fig. 3.9 shows the throughput gain of CAH-MAC over ADHOC MAC in several scenarios. With an increase in $\rho$ and $R$, the number of helpers increases, which ultimately increases the cooperation gain. It is noted that the throughput gains for parameter pairs $\left[R=200, \rho_{l}=30\right]$ and $\left[R=300, \rho_{l}=20\right]$ are the same, because both cases have the same average number of THS members (i.e., $2 \rho R \simeq 24$ ). The throughput gain decreases when the number of THS members is large as compared with $F$. For the parameter pair $[R=300$, 


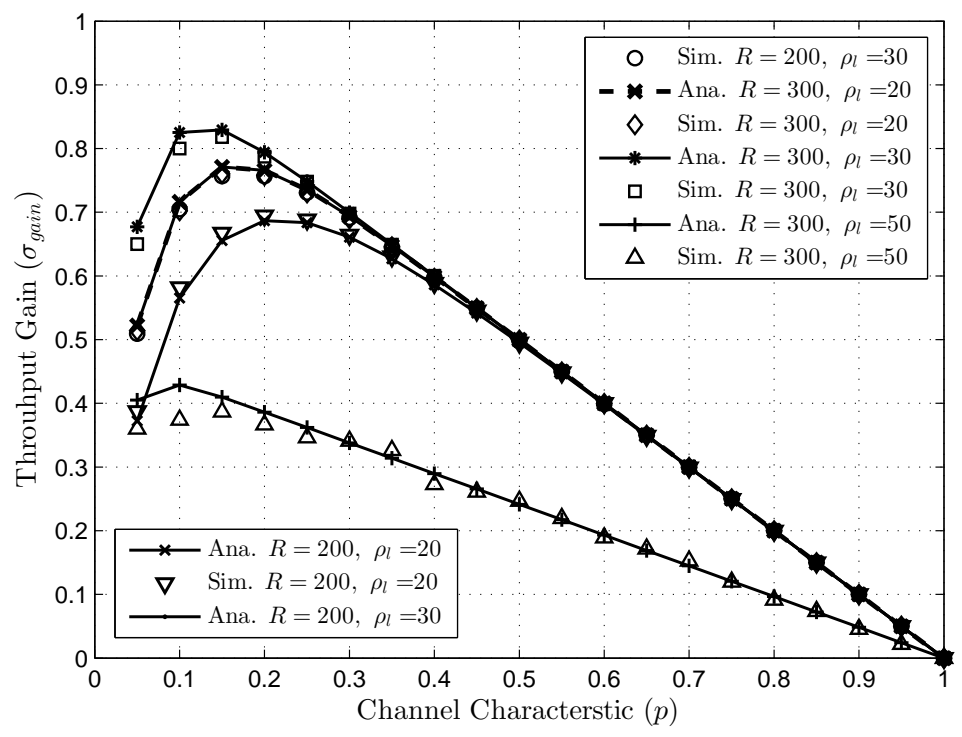

Figure 3.9: Throughput gain of CAH MAC over ADHOC MAC with $F=60$ time slots $\left(\rho_{l}\right.$ is in vehicle per kilometer).

$\left.\rho_{l}=50\right]$, the average number of THS members $(2 \rho R=60)$ is the same as $F$, resulting in a smaller throughput gain than that in the other cases. From Fig. 3.9, it can be seen that the throughput gain reaches its peak at a certain $p$ value and starts decreasing as $p$ further increases. With a large $p$ value, the probability of successful direct transmissions increases and hence cooperation may not be triggered. With a moderate $p$ value, direct transmissions may suffer from channel errors and hence cooperation helps to retransmit the packet that failed to reach the destination. At a small $p$ value, as the helper is likely to suffer from channel errors, the cooperation gain is not significant. Note that the throughput gain increases with an increase in the transmission range and vehicle density when $F \gg 2 \rho R$. As channel characteristics improve (i.e., for $p>0.4$ ), the throughput gain decreases linearly with $p$, irrespective of $R$ and $\rho_{l}$. In such a case, it is very likely that at least one neighboring node successfully receives a packet from the source and there are unreserved time slots for cooperation resulting in $p_{\text {coop }} \simeq 1$. From (3.19), the throughput gain depends only on $p_{s}(=p)$ as $\sigma_{\text {gain }} \simeq 1-p_{s}$. Hence, the throughput gain does not change with $R$ and/or $\rho_{l}$ as long as $F>2 \rho R$, but reduces linearly with $p$. 


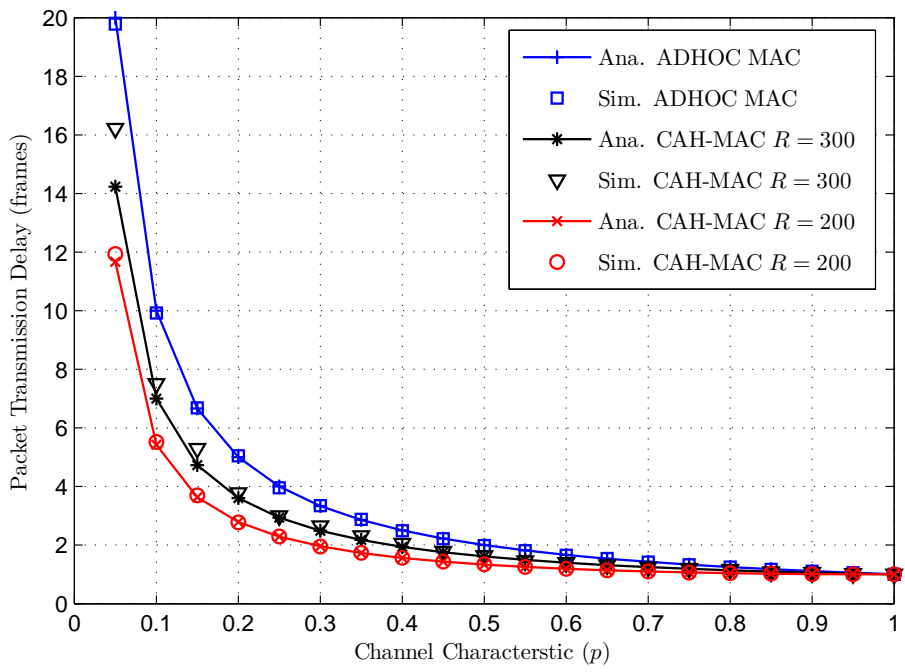

Figure 3.10: Packet transmission delay of ADHOC MAC and CAH-MAC with $\rho_{l}=50$ vehicle per kilometer and $F=60$ time slots.

Fig. 3.10 shows that packet transmission delay of CAH-MAC is almost reduced by $40 \%$ at a poor channel condition (for $p \leq 0.25$ ) as compared with that of ADHOC MAC when $R=200$. However, for the case $R=300$, reduction in packet transmission delay is only about $20 \%$. This is due to the fact that, the advantage of cooperation can be achieved when there are a moderate number of THS members as compared with $F$. In such a case, there are potential helpers and sufficient unreserved time slots to perform cooperative relay transmission. When $R=300$, the average number of THS nodes sharing a frame is almost equal to the total available time slots. i.e., $(2 \rho R=60)$, hence few unreserved time slots. The smaller the number of unreserved time slots or the larger the number of THS members, the smaller the $p_{\text {coop }}$ value, which decreases the cooperation gain. Hence, a less number of unreserved time slots for the cooperative relay transmission results in a higher packet transmission delay for $R=200$. As $p$ increases, the delay improvement starts to decrease. The probability of a successful direct transmission increases with the improvement in the channel condition (i.e., $p \geq 0.85$ ), the gap between packet transmission delay for both protocols decreases as the channel condition deteriorates. 


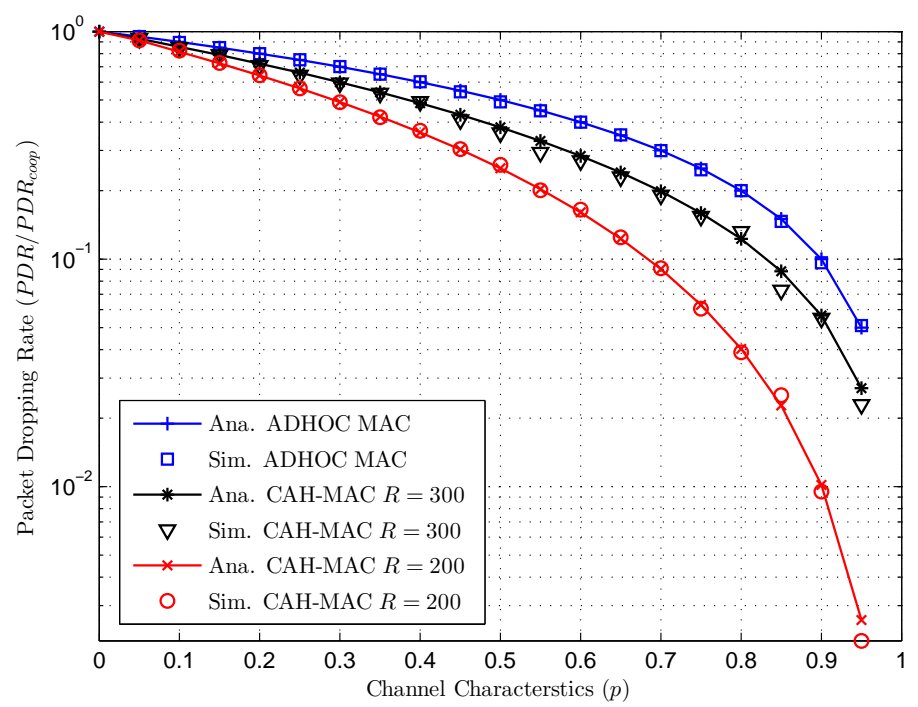

Figure 3.11: Packet dropping rate of ADHOC MAC and CAH-MAC with $M_{\max }=1$ frame, $\rho_{l}=30$ vehicle per kilometer and $F=60$ time slots.

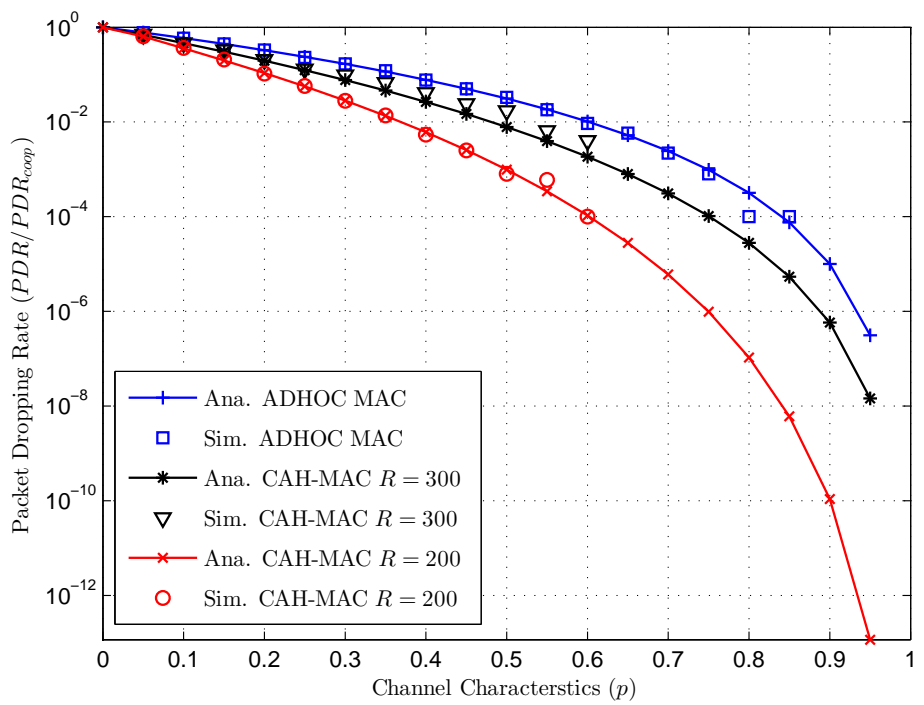

Figure 3.12: Packet dropping rate of ADHOC MAC and CAH-MAC with $M_{\max }=5$ frames, $\rho_{l}=30$ vehicle per kilometer and $F=60$ time slots. 


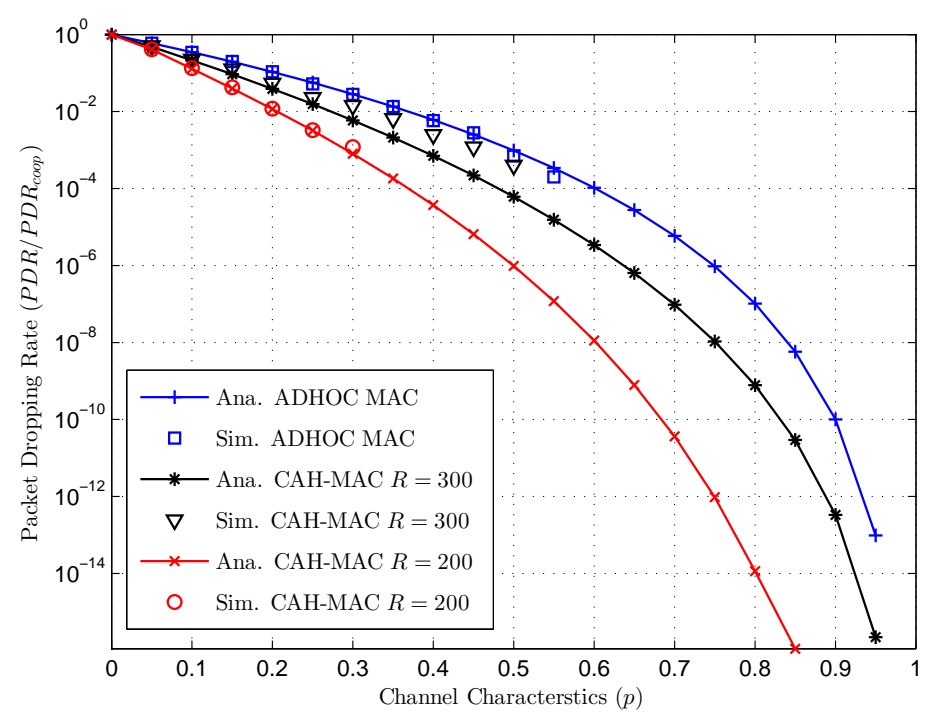

Figure 3.13: Packet dropping rate of ADHOC MAC and CAH-MAC with $M_{\max }=10$ frames, $\rho_{l}=30$ vehicle per kilometer and $F=60$ time slots.

Figs. 3.11-3.13 show packet dropping rate for both protocols at various $M_{\max }$ values. It is observed that the packet dropping rate of CAH-MAC is always less than that of ADHOC $\mathrm{MAC}$ for a given channel condition. However, the gap between the packet dropping rate for $\mathrm{CAH}-\mathrm{MAC}$ and ADHOC MAC increases as the channel gets better in a similar networking condition. Also, the gap increases with the probability of cooperative relay transmission $p_{\text {coop }}$. Hence, the gap between two protocols when $R=200$ is higher than that when $R=300$. For the same channel condition, the larger the $M_{\max }$ value, the larger the gap between the packet dropping rate of two protocols. Upon a transmission failure, in CAH-MAC, the helper node retransmits a packet in an unreserved time slot. Hence, with a larger $M_{\text {max }}$, a node in CAH-MAC gets more retransmission attempts than that of ADHOC MAC. This increases the probability of successful packet delivery to the destination within $M_{\text {max }}$ frames, preventing it from being dropped from the buffer memory. 


\subsection{Summary}

In this chapter, we present a cooperative MAC layer protocol, referred to as CAH-MAC, for VANETs. In CAH-MAC, neighboring nodes cooperate by utilizing unreserved time slots, for retransmission of a packet which failed to reach the target receiver due to a poor channel condition. Through mathematical analysis and simulation, we show that CAH-MAC increases the successful packet transmission probability. Accordingly, system throughput and transmission reliability improve due to cooperative relay transmission. In this chapter, we have not considered relative mobility among nodes. Effects of dynamic network topology changes due to the relative mobility and a more realistic link model on the performance of CAH-MAC are discussed in the next chapter. 


\section{Chapter 4}

\section{Enhanced Node Cooperation}

The previous chapter describes cooperative ADHOC MAC (CAH-MAC) for VANETs that exploits benefits of node cooperation for D-TDMA MAC. Performance evaluation of the CAH-MAC were carried out for a case with negligible relative mobility among nodes. With the mobility among and channel fading, transmission failures occur, due to the transmission collisions and/or channel error, which force a node to release its time slot and seek for a new one (to be discussed). Conflicts occur, in the form of cooperation collisions, if the selected unreserved time slot is chosen by a node seeking a time slot. Thus, cooperation opportunities and time slot reservation attempts fail, and disruption in D-TDMA MAC's operations occurs due to cooperation collisions. To tackle this problem, the existing node cooperation scheme must be improvised to avoid cooperation collisions, thus efficiently utilizing unreserved time slots for either cooperative relay transmissions or time slot reservations.

In this chapter, we present an improvised CAH-MAC with a collision avoidance scheme, referred to as enhanced CAH-MAC (eCAH-MAC) [62, 63]. In eCAH-MAC, cooperative relay transmission is suspended if there is (are) any transmission attempt(s) from the one-hop node(s) of the destination and/or helper nodes, avoiding cooperation collision. Cooperative relay transmission is performed only if the destination and helper nodes do not detect any potential transmissions in their one-hop neighborhood.

Through mathematical analysis and simulations, we show that the proposed collision avoidance scheme increases the utilization of unreserved time slots by either allowing them to 
be reserved by nodes seeking their own time slots or using them to perform cooperative relay transmissions, without disrupting the D-TDMA MAC's normal operations. Furthermore, through extensive simulations we study the performance of eCAH-MAC. A real highway is replicated using PTV VISSIM [73], a microscopic multi-modal traffic flow simulator, to generate vehicle mobility traces. Such mobility traces are used to simulate and evaluate the performance of the newly proposed eCAH-MAC, in comparison with CAH-MAC and ADHOC MAC. Next, necessary assumptions, mainly concerning the transmission channel and time slot access, for tractability in establishing the analytical framework are discussed.

\subsection{System Overview}

\subsubsection{Channel Model}

As the Nakagami- $m$ channel model represents small scale fading in vehicular communication and reflects a realistic driving environment [74], we consider a generalized Nakagami- $m$ channel with correlated amplitudes. For the Nakagami- $m$ channel, the probability density function (pdf) of the received power by a node at $r$ meters from a transmitting node, $\gamma_{r}$, follows a gamma distribution and is given by [75]

$$
f_{\gamma_{r}}(x)=\left(\frac{m}{\bar{\gamma}_{r}}\right)^{m} \frac{x^{m-1}}{\Gamma(m)} e^{\left(-m \frac{x}{\gamma_{r}}\right)}
$$

where $\Gamma(\cdot)$ is the gamma function, $\bar{\gamma}_{r}=\frac{P_{t} C}{r^{\alpha}}$ is the average received power at distance $r$ from the transmitting node, $P_{t}$ is the transmission power, $\alpha$ is the path-loss exponent,

$C=G_{t} G_{r}\left(\frac{c}{4 \pi f_{c}}\right)^{2}$ is a constant, $G_{t}$ and $G_{r}$ are antenna gains at the transmitter and receiver respectively, $f_{c}=5.9 \mathrm{GHz}$ is the carrier frequency, $c=3 \times 10^{8} \mathrm{~m} / \mathrm{s}$ is the speed of light, and $m$ is a distance dependent shape parameter of the Nakagami- $m$ channel, which is 
given as [76]

$$
m= \begin{cases}3, & r \leq 50 \\ 1.5, & 50<r \leq 100 \\ 1, & r>100\end{cases}
$$

In the system, vehicles are moving in a one-way road with the same average speed. In [77] it is shown that, when vehicles move relatively in a similar speed, the auto-correlation function can be approximated by Jake's model [78]. Furthermore, in [79] such approximation is validated simulation for a vehicular environment. Hence, the amplitude correlation coefficient of a signal received, denoted as $\varrho$, at two different time instances, separated by $\tau$ seconds, can be realized by Jake's model, given by [80]

$$
\varrho=J_{0}^{2}\left(2 \pi f_{d} \tau\right)
$$

where, $J_{0}(\cdot)$ is the zeroth-order Bessel function of the first kind and $f_{d}$ is the average Doppler spread. The nature of a time-varying channel greatly depends on the normalized fading rates, which is the product of the average Doppler spread and sample time, i.e., $f_{d} \tau$ [81]. On the other hand, the average Doppler spread, $f_{d}$, of the time-variant vehicle-to-vehicle $(\mathrm{V} 2 \mathrm{~V})$ channel depends on the effective speed, $v_{\text {eff }}=\sqrt{v_{r}^{2}+v_{t}^{2}}$, where $v_{r}$ and $v_{t}$ are the velocities of receiver and transmitter of a link. Moreover, $f_{d}$ depends on the driving environment where the receiver and transmitter are traveling such as highway, rural and suburban environments. As mobile and stationary scatterers (e.g., foliage, pedestrians, passing vehicles) are unavoidable, the presence of such scatterers greatly affect the doppler spread [82] and eventually the channel variations. In [1], the authors present an experiment at $5.9 \mathrm{GHz}$ and derive a close-form expression of Doppler spread in terms of the effective velocity and environment dependent parameters specifically for the V2V channel, and is given by

$$
f_{d}=\frac{\theta}{\lambda \sqrt{2}} v_{e f f}+o
$$

where $\theta$ and $o$ are environment dependent parameters of the V2V channel, referred to as 
slope and offset respectively, whose values are given in Table 4.3 for different environments.

\subsubsection{Cooperation Collisions and Release of Time Slots}

In each frame, a node has information to transmit to a target destination node along with the frame information. Thus, in its own time slot, a node transmits a packet that consists of frame information (FI), packet header $(\mathrm{PH})$, payload data, and cyclic redundancy check (CRC), and makes cooperation decisions (as discussed in Section 3.1). A node puts IDs of its OHS nodes, if it successfully received the packet in the previous frame. Hence, successful reception of FIs helps a node to extract its neighborhood information such as IDs of the one-hop neighboring nodes and their corresponding time slots. Also, FI can be used to detect transmission failures due to poor channel conditions and transmission collisions (access and/or merging) as discussed in Section 2.5. A node releases or continues using its time slot based on the frame information (FI) received from its OHS neighbors. A node releases its time slot, if it fails to detect its ID in FIs from at least one of its OHS member nodes. To avoid unnecessary loss of time slots, a node does not use FI from any new one-hop node that was not in its OHS. However, upon receiving FI from new one-hop nodes, the node updates its OHS. Hence, in the network, there are nodes with and/or without their own time slots. Here, nodes without (with) their own time slots and seeking for one are referred to as contending (resident) nodes, as they contend for time slots in (continue to be a resident of) the corresponding two-hop neighborhood.

Due to channel fading and relative mobility, a node can fail to reserve or retain a time slot; hence, contending nodes remain as contending nodes or resident nodes become contending nodes respectively, in the next frame. Furthermore, in the presence of contending nodes, cooperation collisions may occur during unreserved time slots, among reservation packets from contending nodes and cooperative relay transmissions. Hence, with the introduction of node cooperation, cooperation collisions occur in D-TDMA MAC. Under such a situation, both cooperative relay transmission and time slot reservation fail and contending nodes have to wait longer to acquire time slots. On the other hand, most of the time only resident nodes get an opportunity to access the channel for direct and/or cooperative relay transmission. Furthermore, cooperation collisions occur when cooperative relay transmission is performed 
during a time slot belonging to an existing user. For instance, if a helper node chooses an unreserved time slot to help an $s-d$ pair, collision occurs when a node, which owns the selected unreserved time slot, enters the region where the $s-d$ pair and helper node reside. Under such a case, conflict arises between transmission from newly joined node and scheduled cooperative relay transmission.

A node releases its time slot if it suffers from transmission failure or transmission collision. Upon releasing its time slot, the node seeks a new one. Also, a node which is without a time slot prior to joining the network, seeks a time slot after it joins the network. Furthermore, due to the relative mobility, a node may enter a new THS where its neighboring nodes are not aware of its arrival. If the node owns an unreserved time slot, with respect to the new THS, it keeps on using it as there will not be any conflict. However, a collision occurs if the unreserved time slot is selected to perform cooperative relay transmission. Hence, by the end of each frame, a resident node may lose its time slot and/or a contending node may successfully reserve one. Consequently, a frame consists of reserved and unreserved time slots at the beginning of the next frame. The number of reserved (or unreserved) time slots depends on channel quality, relative mobility, and other networking scenarios.

\subsection{Enhanced Cooperative ADHOC MAC}

The cooperation decisions can lead to cooperation collisions resulting in the failure in time slot reservation and cooperative relay transmission. Hence, in this section, we present the approach in the eCAH-MAC scheme to avoid cooperation collisions. In eCAH-MAC, we propose to use different types of packet structure to resolve cooperation collisions. In the following, we present different packet types and a novel collision avoidance scheme for eCAH-MAC.

\subsubsection{Types of Packet Structure}

A resident node transmits a packet in order to exchange its FI and payload data to the nearby nodes during its own time slot. The resident node must deliver the FI to all nodes in its OHS, to continue using its time slot in the next frame, and payload data to the target 
destination. Furthermore, a resident node may transmit a packet during an unreserved time slot to perform cooperative relay transmission. In such a case, the resident node must deliver the payload data to the target destination(s). On the other hand, a contending node transmits a packet during an unreserved time slot to reserve the time slot for accessing the channel. Thus, the contending node must deliver its FI to all the nodes in its OHS, during the selected unreserved time slot, to successfully reserve the time slot. Hence, it is not necessary to transmit the same information, with the same packet structure or fields, for the aforementioned scenarios. Based on the operations, we define three type of packets that a node can transmit during a time slot, as described below:

- General (Type-G) packet: consisting of FI, PH, payload data and CRC, which is transmitted by a resident node to exchange messages to its nearby nodes. A node transmits Type-G packet only during its own time slot to deliver its FI to the OHS nodes and payload data to the target destination(s). Furthermore, the packet may have a cooperation header $(\mathrm{COH})$ if the transmitting node decides to help an $s-d$ pair that failed to exchange a packet during the source node's time slot.

- Reservation (Type-R) packet: consisting of FI, PH and CRC, which is transmitted by contending nodes to perform time slot reservation. A node first reserves a time slot to access the channel using a Type-R packet (without payload data), then starts to transmit a Type-G packet to exchange payload data with its one-hop neighbor node(s) during the acquired time slot.

- Cooperation (Type-C) packet: consisting of a PH, payload data and CRC, which is transmitted by a helper node to perform cooperative relay transmission. As the helper node has its own time slot to transmit Type-G packets, it is not necessary to transmit its FI during cooperative relay transmission.

In addition to these three types of packet, cooperation acknowledgement $(\mathrm{C}-\mathrm{ACK})$ is transmitted by a destination node to start the cooperative relay transmission phase, which consists of the ID of the helper node, as described in Section 3.1. In the following, advantages of using different packet types in eCAH-MAC are discussed. 


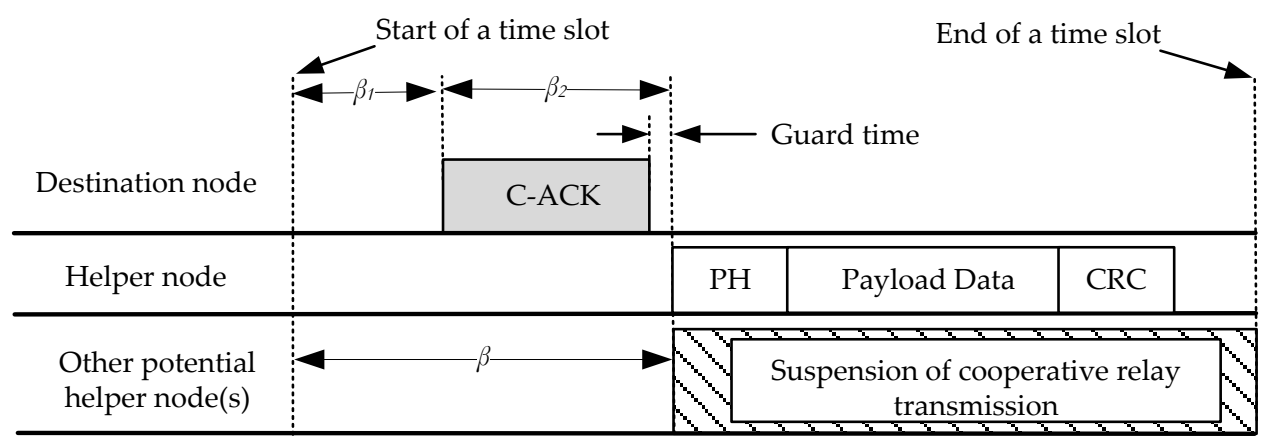

Figure 4.1: Cooperative relay transmission in eCAH-MAC during an unreserved time slot.

\subsubsection{Cooperation Collision Avoidance}

A cooperation collision occurs if an unreserved time slot selected for cooperative relay transmission is accessed by a contending node. One possible way to avoid cooperation collisions is to delay the cooperative relay transmission by some time interval, say $\beta_{1}$ time units. The duration of $\beta_{1}$ units must be long enough for a node to sense whether the channel is idle or busy, such as the distributed inter-frame space (DIFS) in the IEEE 802.11 based MAC protocols [83]. During the selected unreserved time slot, the destination node waits for $\beta_{1}$ time units and then transmits $\mathrm{C}$-ACK if the channel is idle during the waiting time, which is illustrated in Fig. 4.1. Note that in CAH-MAC, the destination node transmits $\mathrm{C}-\mathrm{ACK}$ as soon as the unreserved time slot starts, i.e., $\beta_{1}=0$. The helper node, after receiving its ID in $\mathrm{C}$-ACK from the destination node, transmits a payload data from the source after a guard time. Since the length of C-ACK (in bits) and guard time are constant, the helper node always performs cooperative relay transmission after the fixed duration from the start of a time slot, i.e., $\beta=\beta_{1}+\beta_{2}$ time units as in Fig. 4.1, where $\beta_{2}$ time units correspond to the transmission time of $\mathrm{C}$-ACK plus the guard time.

A helper node transmits a Type-C packet to perform cooperative relay transmission. As each node has its own normal time slot in which it transmits a complete packet, repeated transmission of the FI during cooperative relay transmission is unnecessary. The absence of FI compensates for the delayed time of cooperative relay transmission phase and does not affect the normal operation of D-TDMA and node cooperation. Hence, the transmission of 


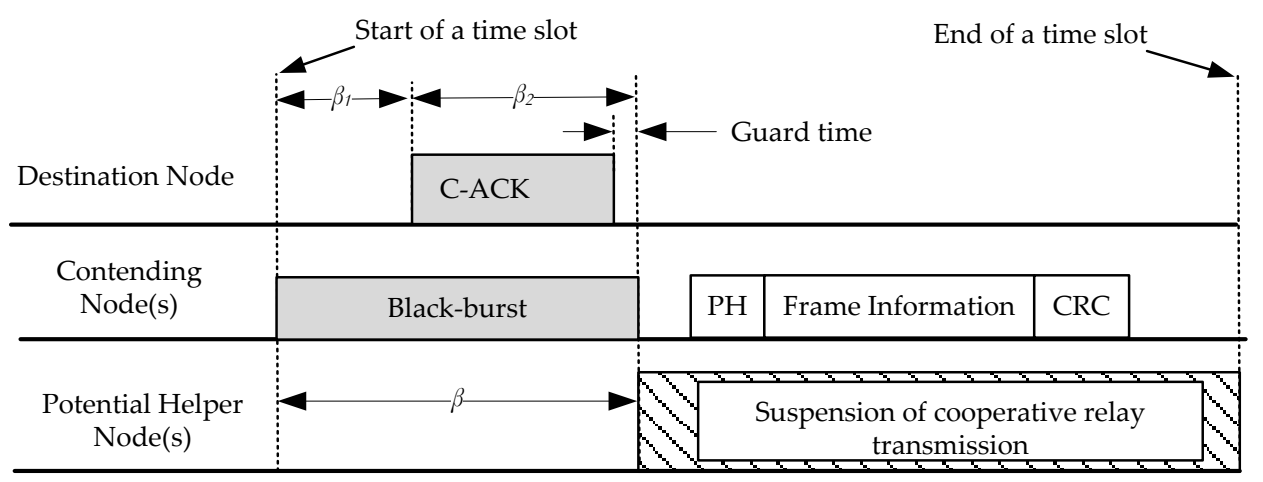

Figure 4.2: Suspension of a cooperative relay transmission by the helper node after failing to receive $\mathrm{C}-\mathrm{ACK}$.

C-ACK and a Type-C packet helps to avoid collision among helper or destination nodes and contending nodes during the cooperative relay transmission.

A contending node may access the unreserved time selected for cooperation. When the destination node detects transmission(s) from the contending node(s), it suspends the cooperation or transmission of the $\mathrm{C}$-ACK. As the helper node does not receive $\mathrm{C}$-ACK, it also suspends cooperative relay transmission. The helper node makes a decision to suspend cooperative relay transmission after $\beta$ time units from the start of a time slot. A collision occurs if a contending node and the destination node are in each others' two-hop distance but not in one-hop distance. In such a case, the destination node does not sense the transmission from the contending node and transmits $\mathrm{C}-\mathrm{ACK}$, resulting in a collision at their common one-hop nodes and/or helper node. To avoid such a collision, both the helper and destination nodes must suspend the cooperative relay transmission. One possible way to force both the destination and helper nodes to suspend cooperation is by using energy-burst or channel jamming signal, also known as black-burst [58]. Black-burst has been used in wireless networks to inform neighboring nodes about the channel usage and to avoid transmission collisions by forcing neighboring nodes to delay or suspend their transmissions $[59,84]$. In eCAH-MAC, a contending node uses black-bursts to inform destination and/or helper nodes about its intention to perform reservation attempt followed by the transmission of a Type-R packet. The contending node transmits a black-burst of $\beta$ time units. If 


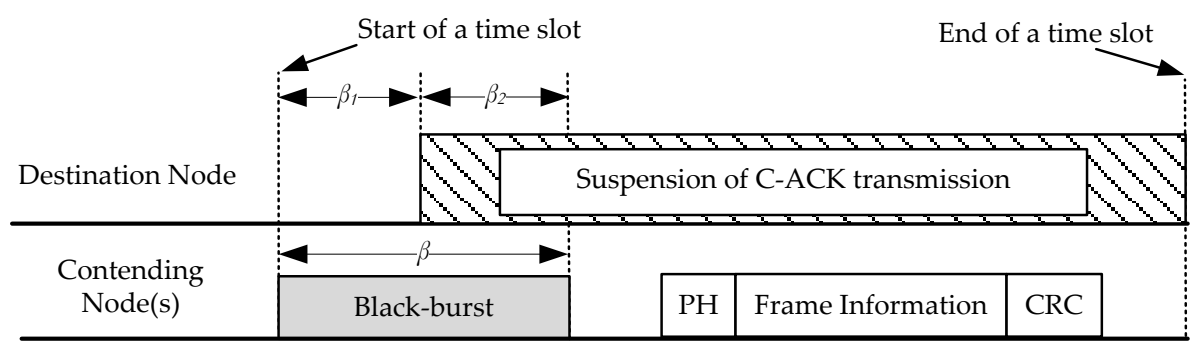

Figure 4.3: Suspension of a cooperative relay transmission by the destination node in the presence of the contending node(s) in its one-hop distance.

the destination node is in the one-hop neighborhood of the contending node, it suspends the cooperative relay transmission after finding the channel busy. On the other hand, if the destination node finds an idle channel, it transmits C-ACK. The transmitted C-ACK collides with the black-burst at helper node's receiver and helper node suspends cooperative relay transmission. After transmitting a black-burst, a contending node transmits a Type$\mathrm{R}$ packet to reserve the corresponding time slot. The suspension of cooperative relay transmission is illustrated in Figs. 4.3 and 4.2. Delaying the cooperative relay transmission phase and the use of a black-burst by contending node allow the destination and/or helper nodes to detect time slot reservation attempts from contending nodes, to avoid cooperation collisions. Access collisions occur if two or more contending nodes transmit black-burst signals and their corresponding Type-R packets during the same unreserved time slot.

Next, we derive a close-form expression for the utilization of an unreserved time slot in eCAH-MAC.

\subsection{Utilization of an Unreserved Time Slot}

Reserved time slots are used in a similar manner in cooperation enabled transmission to that without cooperation. However, with node cooperation enabled, unreserved time slots, if used, are accessed for either time-slot reservations or cooperative relay transmissions. Hence, performance of node cooperation in eCAH-MAC must be evaluated based on how efficiently it utilizes unreserved time slots in comparison with that of D-TDMA MAC, 
such as ADHOC MAC. The objective of such analysis is to study the effectiveness of node cooperation in utilizing unreserved time slots, without affecting the normal operations of D-TDMA MAC. Specifically, we intend to study how an unreserved time slot is utilized in the presence of node cooperation. For tractable analysis, following assumptions are made:

1. A packet is transmitted only once by the source and/or the helper nodes.

2. Cases where a failed direct transmission does not find a helper node and/or unreserved time slot to perform cooperation are ignored. Details of node cooperation and its performance due to the existence of potential helper nodes and unreserved time slots are presented in Chapter 3. Here, we focus on how node cooperation affects time slot reservation, which is one of the important operations in D-TDMA MAC.

3. With a focus on impact of node cooperation in the operation of D-TDMA MAC, only the unreserved time slots chosen to perform cooperative relay transmissions are considered. Time slots that are not chosen for cooperation will not be affected by node cooperation, hence we ignored those cases in the following.

4. We define a parameter $\eta \in(0,1]$, referred to a reserved ratio, which is the ratio of the number of reserved time slots in a frame to the total number of time slots per frame, $F$. The $\eta$ (or 1- $\eta$ ) value reflects the number of reserved (or unreserved) time slots and depends on channel quality, relative mobility, and other networking aspects. At the beginning of a time frame, $\eta F$ time slots are allowed to be reserved. Accordingly, at least $(1-\eta) F$ time slots are unreserved time slots and are left for contending nodes to perform reservation attempts.

To derive a close-form expression for the utilization of unreserved time slots, we consider the following events that can occur during an unreserved time slot selected for cooperative relay transmission:

1. Event $3\left(E_{3}\right)$ : None of the contenting nodes in the THS of the helper and destination nodes sharing a common frame attempts to access the unreserved time slot; 
2. Event $4\left(E_{4}\right)$ : Only one contending node in the THS of the helper and destination nodes sharing a common frame transmits its reservation packet during the selected unreserved time slot.

Next, we derive necessary probability distribution functions required to obtain the probability of the aforementioned events.

\subsubsection{Distribution of Node Number}

Let $N_{C}$ denotes the number of contending nodes in a THS. Contending node exists if the number of THS nodes is greater than $\eta F$, i.e., if $N_{T}>\eta F$, such that $\eta F$ nodes have their own time slots and the remaining are contending nodes seeking their own time slots. Thus, given the reserved ratio, $\eta$, the number of contending nodes in a THS can be written as

$$
N_{C}= \begin{cases}0, & N_{T} \leq \eta F \\ N_{T}-\eta F, & N_{T}>\eta F .\end{cases}
$$

Hence, from (3.2) and (4.5), the pmf of $N_{C}$ can be written as,

$$
\operatorname{Pr}\left\{N_{C}=n_{c}\right\}= \begin{cases}\sum_{n_{t}=0}^{\eta F} \frac{(2 \rho R)^{n_{t}} e^{-2 \rho R}}{n_{t} !}, & \text { if } n_{c}=0 \\ \frac{(2 \rho R)^{\eta F+n_{c}} e^{-2 \rho R}}{\left(\eta F+n_{c}\right) !}, & n_{c}>0 .\end{cases}
$$

The number of unreserved time slot if frame, $U$, is given by

$$
U= \begin{cases}F-N_{T}, & N_{T}<\eta F \\ F-\eta F, & N_{T} \geq \eta F .\end{cases}
$$

Note, a contending node, if exists $\left(N_{C}>0\right.$ or $\left.N_{T}>\eta F\right)$, attempts to transmit a packet during an unreserved time slot. Among $F-\eta F$ available unreserved time slots in a frame, a contending node selects one randomly. Thus, a contending node chooses a given unreserved 
time slot with probability $\frac{1}{F-\eta F}$. Consequently, the probability that a contending node does not choose the given unreserved time slot is $\frac{F-\eta F-1}{F-\eta F}$. Furthermore, if a THS does not contain any contending nodes, i.e., if $N_{C}=0$ or $N_{T} \leq \eta F$, unreserved time slots are not selected to perform reservation attempts. Based on these probability values, next we will derive the probability of occurrence of Events 3 and 4, and use them to derive the required close-form expressions.

\subsubsection{Probability of Event 3}

A contending node performs reservation during an unreserved time slot. Hence, an unreserved time slot remains idle, i.e, Event $E_{3}$ occurs, if none of the contending nodes in the corresponding THS attempt to access it. Based on discussion in the previous subsection, given $N_{C}=n_{c}$, and $N_{T}=n_{t}$, the probability of Event $E_{3}$ occurrences can be written as

$$
\operatorname{Pr}\left\{E_{3} \mid N_{C}=n_{c}, N_{T}=n_{t}\right\}= \begin{cases}1, & n_{t} \leq \eta F \\ \left(\frac{F-\eta F-1}{F-\eta F}\right)^{n_{c}}, & n_{t}>\eta F\end{cases}
$$

Consequently, from (4.5) and (4.8), we have

$$
\operatorname{Pr}\left\{E_{3} \mid N_{C}=n_{c}\right\}= \begin{cases}1, & n_{c}=0 \\ \left(\frac{F-\eta F-1}{F-\eta F}\right)^{n_{c}}, & n_{c}>0 .\end{cases}
$$

From (4.6) and (4.9), the probability that an unreserved time slot is not selected by contending nodes, i.e., the probability of Event 3 occurrences, can be written as

$$
\operatorname{Pr}\left\{E_{3}\right\}=\sum_{n_{t}=0}^{\eta F} \frac{(2 \rho R)^{n_{t}} e^{-2 \rho R}}{n_{t} !}+\sum_{n_{c}>0}\left(\frac{F-\eta F-1}{F-\eta F}\right)^{n_{c}} \frac{(2 \rho R)^{\eta F+n_{c}} e^{-2 \rho R}}{\left(\eta F+n_{c}\right) !}
$$


Which can be further simplified to

$$
\operatorname{Pr}\left\{E_{3}\right\}=\sum_{n_{t}=0}^{\eta F} \frac{(2 \rho R)^{n_{t}} e^{-2 \rho R}}{n_{t} !}+a^{-\eta F} e^{-2 \rho R}\left(e^{b}-\sum_{n_{c}=0}^{\eta F} \frac{b^{n_{c}}}{n_{c} !}\right)
$$

where $a=\frac{F-\eta F-1}{F-\eta F}$ and $b=2 \rho R a$.

\subsubsection{Probability of Event 4}

Event $E_{4}$ occurs if only one of the contending node attempts to access the unreserved time slot selected for node cooperation. Hence, given $N_{C}=n_{c}$ and $N_{T}=n_{t}$, the probability of Event $E_{4}$ occurrences can be written as

$$
\operatorname{Pr}\left\{E_{4} \mid N_{C}=n_{c}, N_{T}=n_{t}\right\}= \begin{cases}0, & n_{t} \leq \eta F \\ n_{c}\left(\frac{1}{F-\eta F}\right)\left(\frac{F-\eta F-1}{F-\eta F}\right)^{n_{c}-1}, & n_{t}>\eta F\end{cases}
$$

Consequently, from (4.5) and (4.12), we have

$$
\operatorname{Pr}\left\{E_{4} \mid N_{C}=n_{c}\right\}= \begin{cases}0, & n_{c}=0 \\ n_{c}\left(\frac{1}{F-\eta F}\right)\left(\frac{F-\eta F-1}{F-\eta F}\right)^{n_{c}-1}, & n_{c}>0\end{cases}
$$

From (4.6) and (4.13), the probability that an unreserved time slot is selected by only one contending node, i.e., the probability of Event 4 occurrences, can be written as

$$
\operatorname{Pr}\left\{E_{4}\right\}=\sum_{n_{c}>0} n_{c}\left(\frac{1}{F-\eta F}\right)\left(\frac{F-\eta F-1}{F-\eta F}\right)^{n_{c}-1} \frac{(2 \rho R)^{\eta F+n_{c}} e^{-2 \rho R}}{\left(\eta F+n_{c}\right) !}
$$

which can be further simplified to

$$
\operatorname{Pr}\left\{E_{4}\right\}=\left(\frac{1-a}{a^{\eta F+1}}\right) e^{-2 \rho R}\left[(b-\eta F) e^{b}+\sum_{n_{c}=0}^{\eta F-1}\left(\eta F-n_{c}\right) \frac{b^{n_{c}}}{n_{c} !}\right] .
$$




\subsubsection{Close-Form Expressions for Time Slot Utilization}

In $\mathrm{ADHOC} \mathrm{MAC}$, as cooperation is not enabled, a successful time slot reservation guarantees the efficient utilization of the unreserved time slot. With cooperation enabled transmission, in addition to successful time slot reservation, successful cooperative relay transmission further guarantees the efficient utilization of unreserved time slots. In the following, we use the probabilities of event occurrences from the previous subsections to derive the utilization of an unreserved time slot for ADHOC MAC, CAH-MAC and eCAH-MAC.

In ADHOC MAC, an unreserved time slot is referred to as efficiently utilized if only one contending node chooses it to perform reservation. In other cases, i.e., if it remains unused or more than one contending nodes transmit their reservation packets, the unreserved time slot is wasted. Hence, the utilization of an unreserved time slot in ADHOC MAC, denoted as $\epsilon_{A}$, can be written as

$$
\epsilon_{A}=\operatorname{Pr}\left\{E_{4}\right\} .
$$

On the other hand, in CAH-MAC, an unreserved time slot selected for cooperation is considered to be efficiently utilized if none of the contending nodes choose it to perform reservation. A cooperation (access) collision occurs if at least one (more than one) contending node chooses to reserve the unreserved time slot selected to perform cooperative relay transmission (time slot reservation). Hence, the utilization of an unreserved time slot in CAH-MAC, denoted as $\epsilon_{C}$, can be written as

$$
\epsilon_{C}=\operatorname{Pr}\left\{E_{3}\right\} .
$$

Finally, in eCAH-MAC, an unreserved time slot selected for cooperation is considered to be efficiently utilized if it is used to perform either cooperative relay transmission, if none of the contending node chooses it, or reservation attempt is performed by only one contending node. Hence, the utilization of an unreserved time slot in eCAH-MAC, denoted as $\epsilon_{E}$, is given by

$$
\epsilon_{E}=\operatorname{Pr}\left\{E_{3}\right\}+\operatorname{Pr}\left\{E_{4}\right\} .
$$


Table 4.1: Parameters used in simulation

\begin{tabular}{|l|l|}
\hline Parameter & Value \\
\hline \hline Path loss exponent $(\alpha)$ & 2,3 and 4 \\
\hline Transmission power $\left(P_{t}\right)$ & $20 \mathrm{~mW}$ \\
\hline Threshold received power $\left(\gamma_{t h}\right)$ & $95 \mathrm{dBm}$ \\
\hline Transmission range $(R)$ & 100 meters \\
\hline Number of time slots per frame $(F)$ & 50 \\
\hline Time slot duration $(\tau)$ & 1 millisecond \\
\hline Simulation time & 120 seconds \\
\hline \hline
\end{tabular}

\subsection{Numerical Results}

In this section, we present numerical results obtained from simulations to analyze the performance of eCAH-MAC. Simulations are performed in MATLAB with parameters given in Table 4.1. We consider free-flow node mobility as in [40] such that, at the beginning of simulation, vehicles are distributed following a Poisson distribution, along a road segment of length 10 kilometers with three lanes ${ }^{1}$ is considered. The speed of vehicles follows a normal distribution ${ }^{2}$, with mean 100 kilometer per hour and standard deviation 20 kilometer per hour. Each vehicle moves with the same speed throughout the simulation. Furthermore, vehicles exiting from one end of the road segment re-enters from the other end. To avoid any unrealistic loss of time slot, a vehicle that is at a distance $r$ from one end of the road segment can communicate with vehicles which are at a distance $R-r$ from the other end, such that vehicles do not break their communications even after exiting from one end of the road segment and entering from the other end. Utilization of eCAH-MAC, CAH-AMC and ADHOC MAC are obtained and compared for several $\eta$ values to validate the close-form expressions for time slot utilization derived in Section 4.3.

Fig. 4.4 compares the utilization with $\eta=0.8,0.5$ and 0.2 , respectively. It can be seen that the analytical results match well with simulation results, thus validating the close-form expressions for utilization of unreserved time slots selected for cooperative relay transmission. For a given average number of two-hop nodes, $\bar{N}_{T}$, the larger the $\eta$ value, the higher the utilization of unreserved time slot. This is obvious as a larger $\eta$ value results

\footnotetext{
${ }^{1} \mathrm{~A}$ line represents a lane that is 5 meters wide. Vehicles are represented by points on the lines.

${ }^{2}$ Negative and zero velocity values are ignored while drawing the speed of a vehicle.
} 


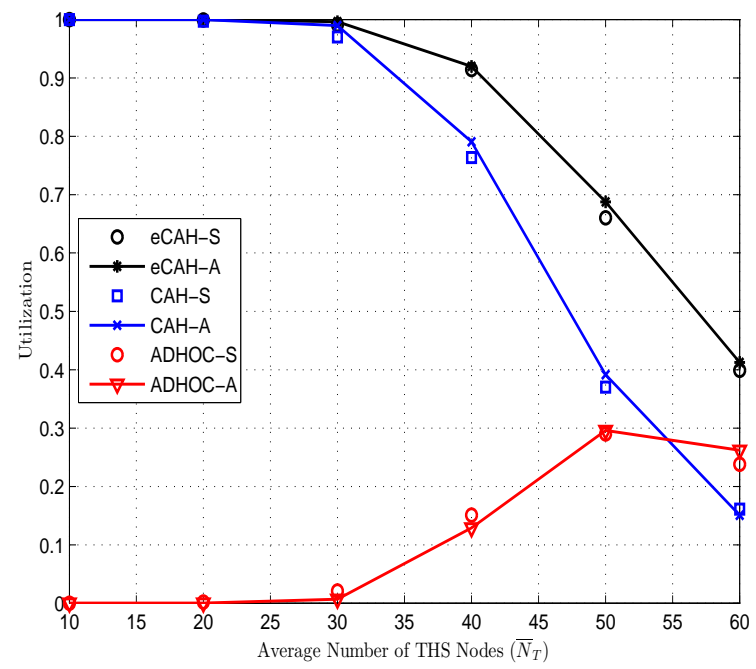

(a)

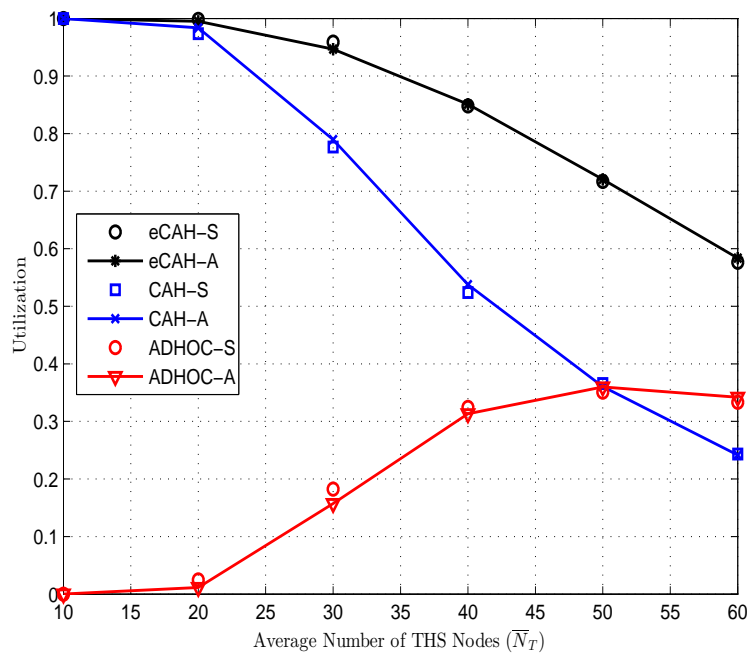

(b)

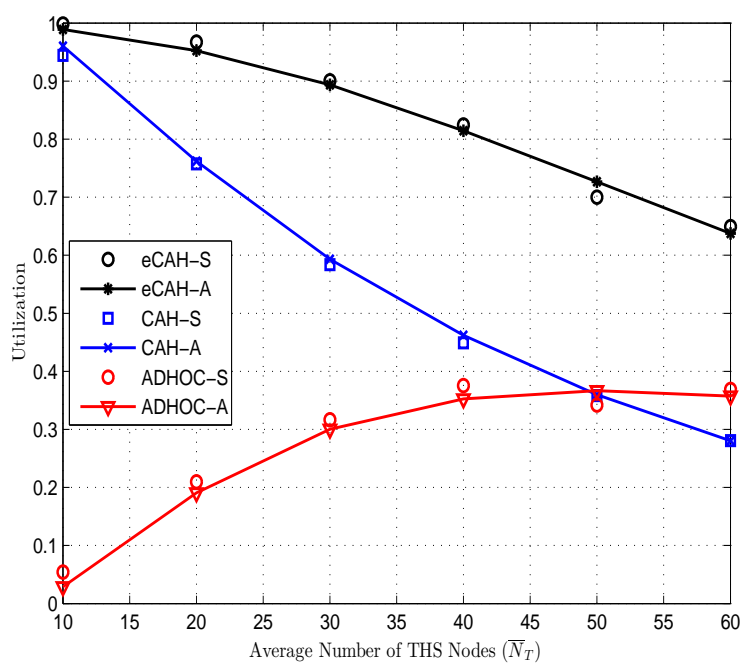

(c)

Figure 4.4: Comparison of utilization of unreserved time slots in eCAH-MAC, CAH-MAC and ADHOC MAC with (a) $\eta=0.80$; (b) $\eta=0.50$; (c) $\eta=0.20$. 
in a lesser number of contending nodes, i.e., a lesser number of reservation attempts and consequently, a lesser number of cooperation collisions. However, for a larger $\bar{N}_{T}$ values, the utilization decreases with an increase in $\eta$ values, for eCAH-MAC and ADHOC MAC. This is because, the lesser the $\eta$ value, the larger the number of contending nodes and unreserved time slots, which results in successful time slot reservation and increases the utilization. As the average number of THS nodes increases, the utilization decreases in cooperation

enabled transmission. As the $\bar{N}_{T}$ value increases, the average number of contending nodes increases, increasing the number of reservation attempts. Such reservation attempts improve the performance of ADHOC MAC and eCAH-MAC (as it is used to reserve a time slot). However, in CAH-MAC, it decreases the performance due to cooperation collisions. When the average number of THS nodes is high, CAH-MAC performs worst among all the D-TDMA MAC (e.g., $\bar{N}_{T}>50$ in both the cases). This is because, at a high $\bar{N}_{T}$ value, reservation attempts by a contending node during an unreserved time slot will fail due to cooperative relay transmission. Thus, it reduces the utilization value as both time slot access and cooperative relay transmission fail.

\subsection{Simulation Results}

Computer simulations with vehicle mobility traces and Nakagami- $m$ channel model are conducted to evaluate the performance of eCAH-MAC and compare it with that of CAHMAC and ADHOC MAC. To simulate mobility among nodes, we use a well-known vehicle traffic simulator PTV VISSIM [73] and MATLAB. Real road networks are replicated in VISSIM to generate vehicle traces. We consider a road network based on a segment of Highway 401 of the province of Ontario in Canada. To keep vehicles in the simulation, a ring of highway segment, of length approximately 4 kilometers with three lanes, is formed, such that the vehicles keep moving in the ring and do not escape throughout the simulation. Road segments, speed limits, and other traffic rules are defined based on realistic observations. Vehicles such as cars, heavy goods vehicles (HGVs) and buses are included. Vehicles follow the Wiedemann99 Car Following Model [85] to follow the headway traffic. Based on these models, appropriate decisions are made to perform lane changes, left or right turns, and to follow the vehicle in front. 
Table 4.2: VISSIM simulation parameters

\begin{tabular}{|c|c|c|c|c|c|c|c|c|c|c|}
\hline \multicolumn{4}{|c|}{$\begin{array}{c}\text { Car-following } \\
\text { (Wiedemann99) }\end{array}$} & \multicolumn{3}{|c|}{ Lane changing } & \multicolumn{4}{|c|}{ Vehicle characteristics } \\
\hline $\begin{array}{l}\text { Para- } \\
\text { meter }\end{array}$ & $\begin{array}{l}\text { Val- } \\
u e\end{array}$ & $\begin{array}{l}\text { Para- } \\
\text { meter }\end{array}$ & $\begin{array}{l}\text { Val- } \\
\text { ue }\end{array}$ & $\begin{array}{l}\text { Para- } \\
\text { meter }\end{array}$ & $\begin{array}{c}\text { Lane } \\
\text { changer }\end{array}$ & $\begin{array}{l}\text { Trailing } \\
\text { vehicle }\end{array}$ & $\begin{array}{l}\text { Para- } \\
\text { meter }\end{array}$ & Car & $H G V$ & Bus \\
\hline $\mathrm{CC} 0$ & $\begin{array}{c}1.5 \\
\mathrm{~m}\end{array}$ & $\mathrm{CC} 1$ & $\begin{array}{c}0.90 \\
\mathrm{~m}\end{array}$ & $\begin{array}{l}\text { Maximum } \\
\text { decelera- } \\
\text { tion }\end{array}$ & $-4 \mathrm{~m} / \mathrm{s}^{2}$ & $-3 \mathrm{~m} / \mathrm{s}^{2}$ & $\begin{array}{l}\text { Average } \\
\text { length }\end{array}$ & $\begin{array}{c}4.44 \\
\mathrm{~m}\end{array}$ & $\begin{array}{c}11.54 \\
\mathrm{~m}\end{array}$ & $\begin{array}{c}11.54 \\
\mathrm{~m}\end{array}$ \\
\hline $\mathrm{CC} 2$ & $\begin{array}{l}4.0 \\
\mathrm{~m}\end{array}$ & $\mathrm{CC} 3$ & -8.0 & $\begin{array}{c}-1 \mathrm{~m} / \mathrm{s}^{2} \\
\text { per } \\
\text { distance }\end{array}$ & $200 \mathrm{~m}$ & $200 \mathrm{~m}$ & Width & $\begin{array}{c}1.5 \\
\mathrm{~m}\end{array}$ & $\begin{array}{c}2.5 \\
\mathrm{~m}\end{array}$ & 2.5 \\
\hline $\mathrm{CC} 4$ & $\begin{array}{c}- \\
0.35\end{array}$ & CC5 & 0.35 & $\begin{array}{c}\text { Accepted } \\
\text { decelera- } \\
\text { tion }\end{array}$ & $-1 \mathrm{~m} / \mathrm{s}^{2}$ & $\begin{array}{l}-0.50 \\
\mathrm{~m} / \mathrm{s}^{2}\end{array}$ & $\begin{array}{c}\% \text { of } \\
\text { the } \\
\text { total \# } \\
\text { of vehi- } \\
\text { cles }\end{array}$ & $95 \%$ & $15 \%$ & $5 \%$ \\
\hline CC6 & 11.44 & $\mathrm{CC} 7$ & $\begin{array}{l}0.25 \\
\mathrm{~m} / \mathrm{s}^{2}\end{array}$ & Simulati & on time: 5 & minutes & $\begin{array}{c}\text { Desire } \\
\text { speed } \\
\text { dist. } \\
(\mathrm{km} / \mathrm{h})\end{array}$ & $\begin{array}{l}\mathrm{U}(90, \\
130)\end{array}$ & $\begin{array}{l}\mathrm{U}(85 \\
120)\end{array}$ & $\begin{array}{l}\mathrm{U}(85, \\
120)\end{array}$ \\
\hline $\mathrm{CC} 8$ & $\begin{array}{c}3.5 \\
\mathrm{~m} / \mathrm{s}^{2}\end{array}$ & CC9 & $\begin{array}{l}1.50 \\
\mathrm{~m} / \mathrm{s}^{2}\end{array}$ & $\begin{array}{l}\text { Units in m. } \\
\text { VISSIM pal } \\
\text { cars, HGVs }\end{array}$ & $\begin{array}{l}\text { and secon } \\
\text { ters such a } \\
\text { buses are }\end{array}$ & $\begin{array}{l}\text { denoted b } \\
\text { imum/des }\end{array}$ & $\begin{array}{l}\text { ind } \mathrm{s}, \mathrm{r} \\
\text { ccelerat } \\
\text { c73]. }\end{array}$ & vely. & $\begin{array}{l}\text { thermc } \\
\text { tion fu }\end{array}$ & $\begin{array}{l}\text { default } \\
\text { tions for }\end{array}$ \\
\hline
\end{tabular}

At the start of simulations, vehicles are injected to the road networks with rate 2100 , 2400 and 7200 vehicle per hour. After the injection period of 5 minutes, the vehicle injection is stopped and the number of vehicles in the network, denoted as $\aleph$, becomes 364, 496 and 622 , respectively, for each vehicle input rate. The generated vehicles are allowed to move according to the corresponding traffic rules and road network parameters. To reduce any transient state effects, vehicle traces are not recorded for a warm-up period of 5 minutes (after the injection period). When the warm-up period ends, vehicle traces are recorded at the interval of 0.1 second till the end of simulation. The simulation time is the time interval between the end of warm-up period and end of simulation, i.e., actual start of simulation is considered only after the end of warm-up period. The generated vehicle traces consist of vehicle positions, i.e., $x$ and $y$ coordinates, and speeds at a given time. Such vehicle traces are used to simulate the performance of eCAH-MAC, which is compared with that of CAH-MAC and ADHOC MAC. The VISSIM simulation parameters are given in Table 4.2. 
Table 4.3: Parameters for doppler spread in different driving environments[1]

\begin{tabular}{|l|c|c|c|}
\hline Parameter & Rural & Highway & Suburban \\
\hline \hline Offset $(o)$ & 0.500 & 0.200 & 11.20 \\
\hline Slope $(\theta)$ & 0.420 & 0.414 & 0.428 \\
\hline \hline
\end{tabular}

To realize the channel, autocorrelated Nakagami- $m$ envelope sequences are generated based on [86], such that the generated sequence follows the rank statistics of the reference Rayleigh sequence that is generated based on Jake model [78]. Correlation coefficient is generated as in (4.3) and (4.4), with slope and offset for highway environment as in Table 4.3. Other simulation parameters are given in Table 4.1.

Fig. 4.5 shows the fractions of resident and contending nodes, and reserved and unreserved time slots in a THS sharing a common frame, for various channel conditions. As the channel degrades, a large number of nodes lose their time slots and become contending nodes. Consequently, more time slots are left unreserved in the corresponding frame. Also, Fig. 4.5(c) shows the frame-by-frame status of time slots, during a stable-state after the initial transitions. At the beginning of the simulation, all nodes in the network try to access the channel, which results in collision. In the steady state, for a given networking scenario, the fractions of reserved (unreserved) time slot remains constant at each time frame.

To study the effects of node cooperation on the operations of D-TDMA MAC, the performance of eCAH-MAC is evaluated based on how efficiently an unreserved time slot selected for cooperative relay transmission is used. To do so, we ignore unreserved time slots during which $(i)$ two or more contending nodes perform reservation attempts, and (ii) cooperative relay transmission is not scheduled for a given failed direct transmission. In such events, irrespective of the presence of node cooperation, reservation attempts are unsuccessful due to access collisions or not affected by node cooperation, respectively. If a cooperation is scheduled during an unreserved time slot, one of the following events occur ( $i$ ) a helper node successfully relays the packet to the destination node, i.e., a successful cooperative relay transmission; ( $i i)$ a helper node fails to relay the packet to the destination node due to a poor channel, i.e., a failed cooperative relay transmission due to poor channel condition; and, ( iii) cooperative relay transmission collides or gets suspended due to the reservation attempt from a contending node that is in one-hop neighborhood from either destination node or helper node. Fig. 4.6 shows the probabilities of such events. As the 


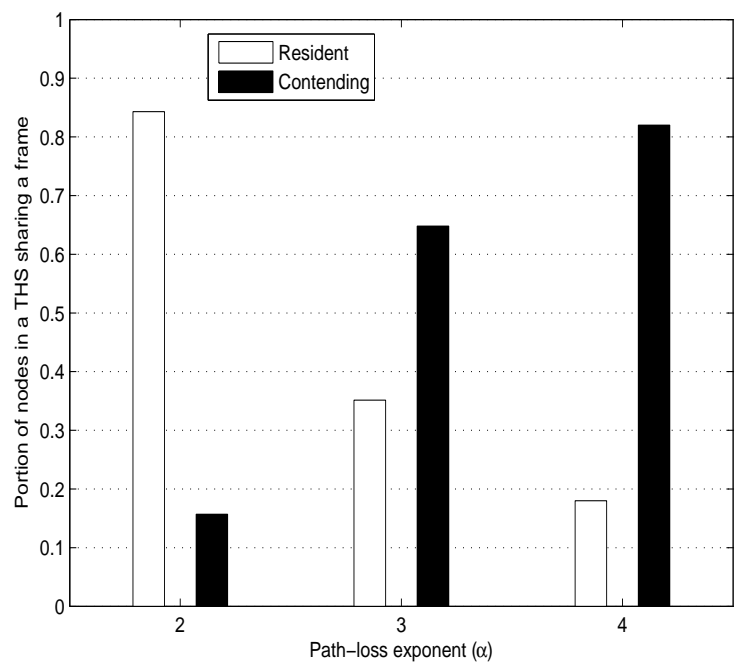

(a)

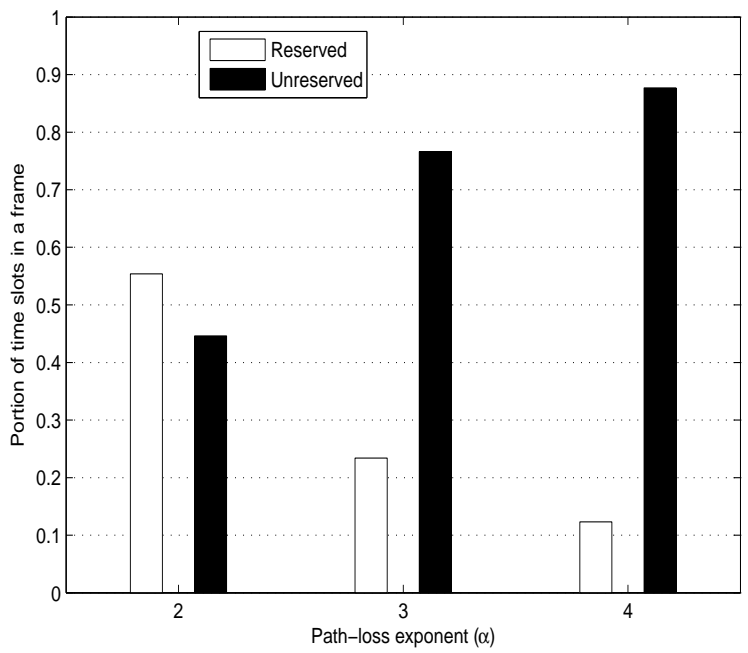

(b)

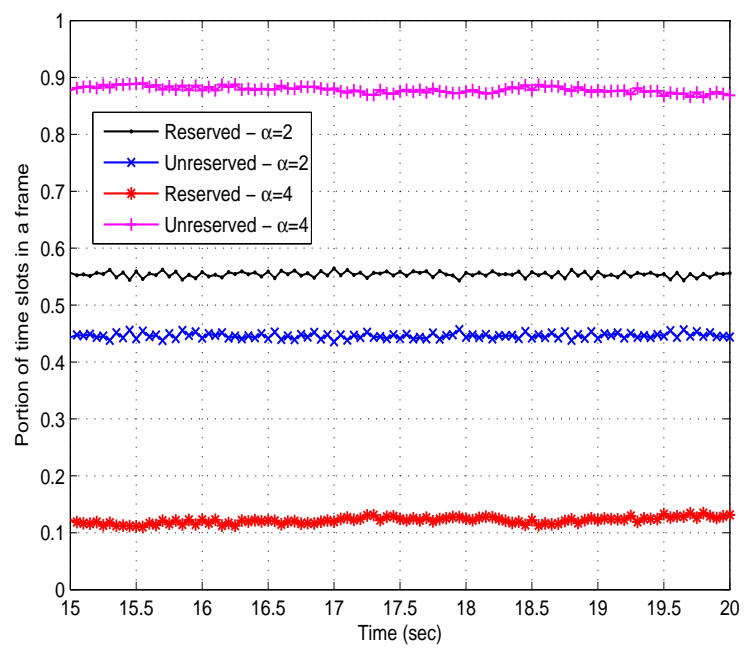

(c)

Figure 4.5: Status of two-hop member nodes and time slots of the corresponding frame with $\aleph=622$ over various channel conditions: (a) Portion of the number of resident and contending nodes in a THS per frame; (b) Portion of reserved and unreserved time slots in a THS per frame; (c) Portion of reserved and unreserved time slots in a THS per frame observed between consecutive frames in a steady state. 


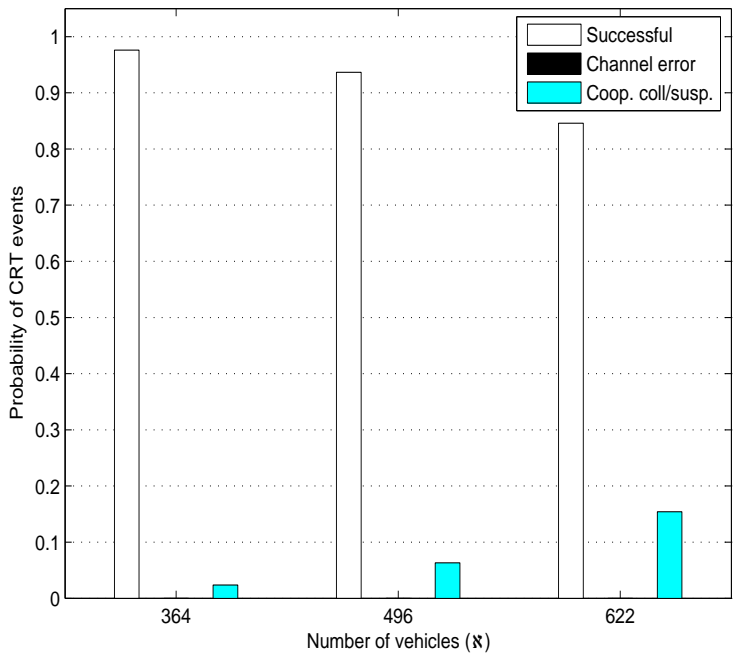

(a)

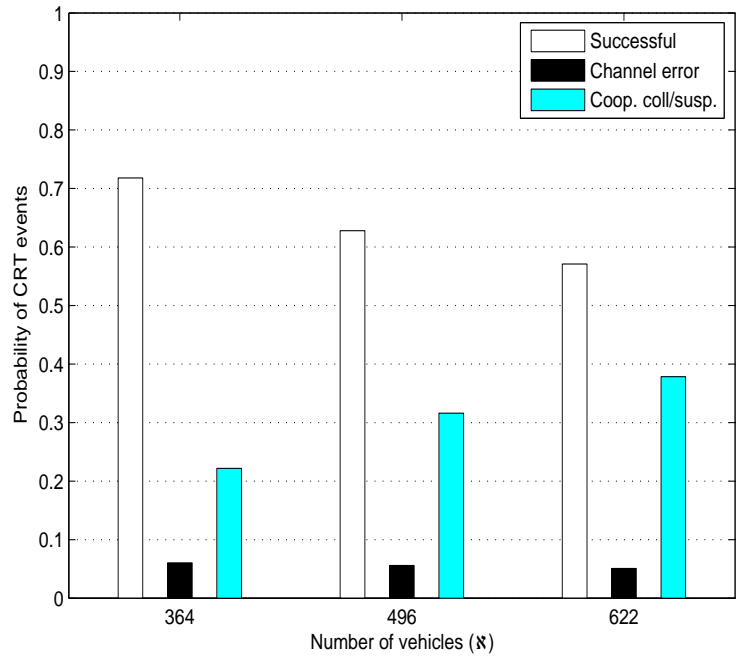

(b)

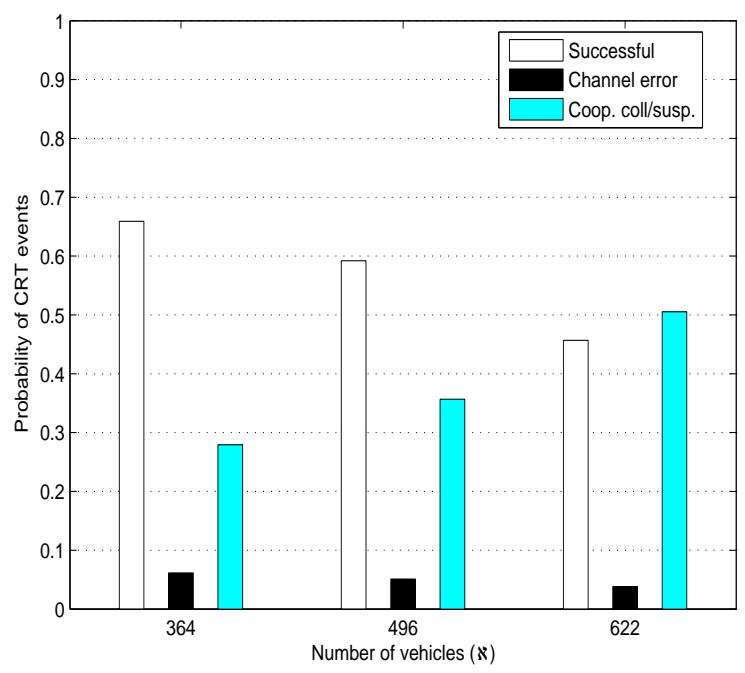

(c)

Figure 4.6: Probability of events during cooperative relay transmission (CRT), such as successful CRT (Successful), failed CRT due to channel error (Channel error), and failed CRT due to cooperation collision in CAH-MAC or suspension of CRT to avoid cooperation collision in eCAH-MAC (Coop. coll/susp.), with (a) $\alpha=2$; (b) $\alpha=3$; (c) $\alpha=4$. 
number of nodes in the network increases or the channel quality degrades (when $\alpha$ value increases), the probability of successful cooperative relay transmission decreases. This is primarily due to an increase in the number of cooperation collisions in eCAH-MAC or suspension of cooperative relay transmissions, as a large number of nodes lose their time slots due to channel errors. Similarly, with an increase in the number of nodes in the network, for a given $F$ value, the number of contending node increases. The larger the contending nodes, the higher the probability of reservation attempt(s) during an unreserved time slot. Thus, such phenomenon forces helper and/or destination nodes to suspend the scheduled cooperative relay transmissions in eCAH-MAC or results in cooperation collisions in CAH-MAC, decreasing the probability of successful cooperative relay transmission. In CAH-MAC, cooperation collisions occur when a helper and a new node simultaneously perform cooperative relay transmission and time slot reservation, whereas in eCAH-MAC the destination node suspends the cooperative relay transmission phase when it detects a reservation packet(s) from the contending node(s).

Furthermore, we study the effectiveness of node cooperation in efficiently using the idle time slots that are selected for node cooperation. Fig. 4.7 shows the probability of using an unreserved time slot in ADHOC MAC, CAH-MAC, and eCAH-MAC. As the number of nodes increases in the network, the average distance between neighboring nodes decreases. As a result, the capability of a contending node to successfully transmit its reservation packet to all of its OHS nodes due to a poor channel condition decreases. Further, in CAH-MAC, the ongoing cooperative relay transmissions lead to cooperation collisions, wasting time slot. On the other hand, cooperative relay transmission is suspended in eCAH-MAC, allowing contending nodes to perform reservation attempts. Hence, eCAH-MAC uses an unreserved time slot better than CAH-MAC and ADHOC MAC.

\subsection{Summary}

In this chapter, we present a collision avoidance scheme for the CAH-MAC protocol, referred to as enhanced Cooperative ADHOC MAC (eCAH-MAC). In the presence of dynamic networking environment, CAH-MAC suffers from cooperation collisions between cooperative relay transmissions and time slot reservations. In eCAH-MAC, the use of three packet 


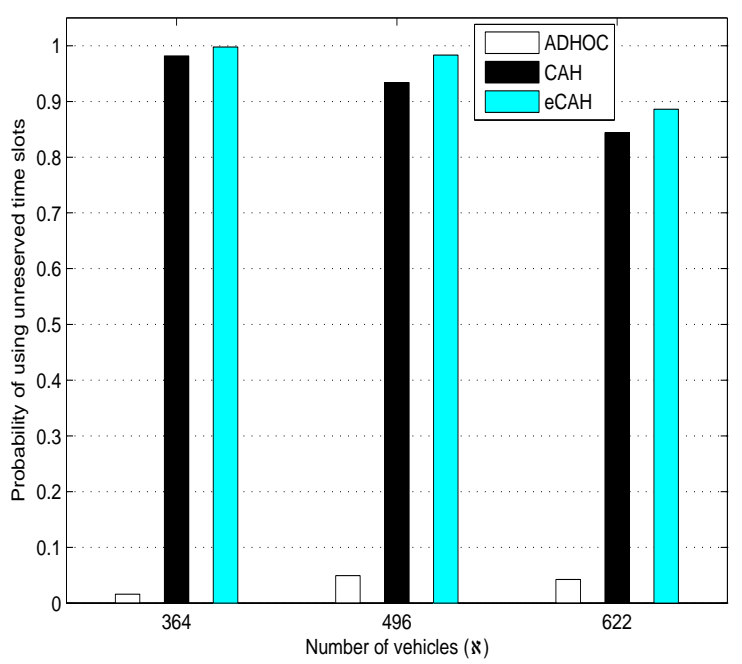

(a)

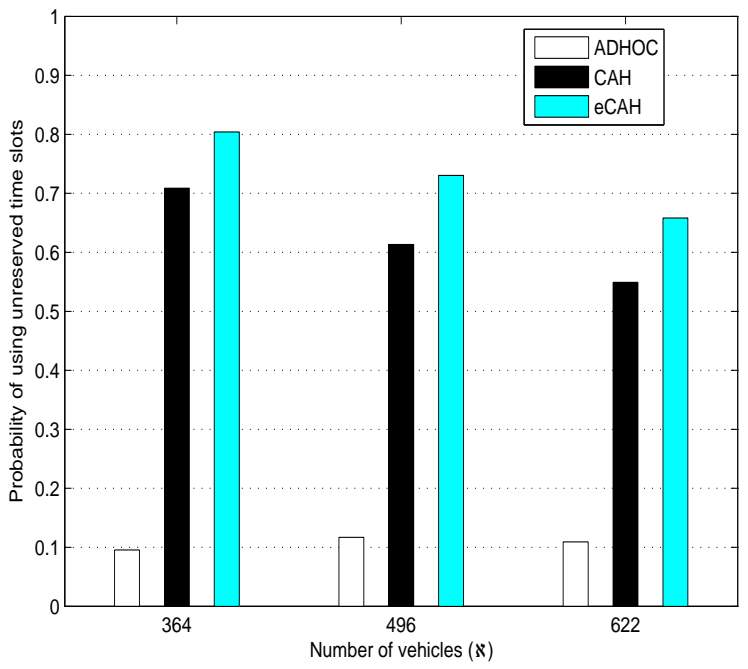

(b)

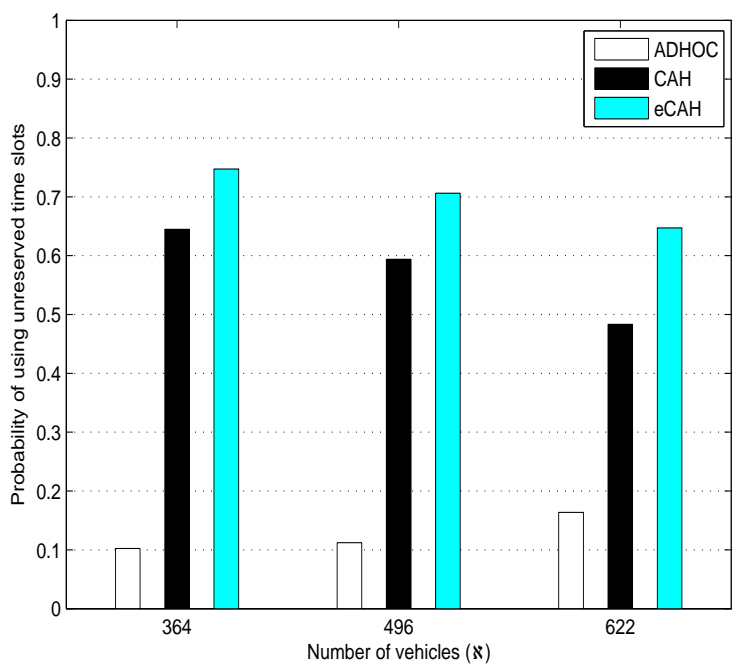

(c)

Figure 4.7: Probability of successful usage of unreserved time slots in ADHOC MAC, CAH-MAC and eCAH-MAC, with (a) $\alpha=2$; (b) $\alpha=3$; (c) $\alpha=4$. 
types, use of black bursts to reserve a time slot, and suspension and/or delay of cooperative relay transmission phase allow to avoid cooperation collision and, thus, efficiently use unreserved time slots either to perform cooperative relay transmissions or new time slot reservations. Through extensive simulation and mathematical analysis, we observe that in eCAH-MAC unreserved time slots are used efficiently either to perform time slot reservation or cooperative relay transmission without interfering each other. 


\section{Chapter 5}

\section{Cooperative Relay Broadcasting}

In this chapter, we present node cooperation based makeup transmission framework for D-TDMA MAC in VANETs, referred to as cooperative relay broadcasting (CRB) [64]. In $\mathrm{CRB}$, nodes with packet from a source node rebroadcast the packet, making it suitable for delay sensitive safety applications with strict QoS requirements. As discussed in Section 1.1, delay sensitive safety applications demand an efficient, quick, and reliable broadcast service. However, the existing D-TDMA MAC protocol lack a makeup strategy to tackle a transmission failure due to the wireless channel impairments in VANETs. The lack of a makeup strategy in D-TDMA MAC results in the following problems in broadcast services:

1. To rebroadcast a safety packet, a source node must analyze its neighborhood information, namely frame information (FI) from all of its one-hop neighbors, which takes the duration of a time frame. This results in an undesirable packet transmission delay, and may result in packet dropping if the packet expiry time limit is exceeded;

2. Upon a transmission failure, a source node waits for its own time slot in the next frame, to rebroadcast the packet even if there are unused time slots before its time slot in the next frame. This results in a delay although the channel is idle during the unused time slots. Unused time slots can be used to rebroadcast the packet (before source node's next transmission) to reduce the packet delay;

3. Retransmission from a source node may not always be helpful to successfully deliver a 
packet that failed to reach some of its one-hop neighbors. For example, the presence of a large obstacle (such as a large vehicle) in between two vehicles may lead to frequent link breakage and failure in transmission even after multiple attempts [87].

Hence, the required QoS may not be achieved if the source node rebroadcasts the packet. As a result, it is necessary for the D-TDMA MAC to have a makeup strategy that provides a quick and reliable one-hop broadcast service specifically to satisfy the strict QoS requirements of the delay sensitive safety applications.

Node cooperation schemes such as CAH-MAC and eCAH-MAC, as discussed in Chapters 3 and 4 respectively, deploy a makeup strategy for a point-to-point communication in DTDMA MAC. However, to the best of our knowledge a makeup strategy for broadcast service in D-TDMA based MAC is not yet available. Furthermore, the existing makeup strategies for broadcast service in wireless networks, as discussed in subsection 1.3.2, cannot be directly applied to D-TDMA. Motivated from the lack of a suitable makeup strategy, in this chapter, we focus on developing a framework for makeup transmission in D-TDMA based broadcast service in VANETs, which supports safety applications demanding a quick and reliable broadcast service. The nodes with a valid packet (broadcast from a source node) cooperate with the remaining nodes to ensure that a maximum number of nodes receive the packet before it expires. The key contributions of this chapter are as follows:

1. We propose a framework that allows cooperative relay broadcasting (CRB) in DTDMA to make up for the transmission failures during the source node's time slot, using the available unused time slots. The CRB is performed by nodes, referred to as the best helper nodes, which are in source node's transmission range and have a good channel condition to the nodes which failed to receive the packet from the source node. The main objective of the proposed CRB framework is to maximize the number of nodes which successfully receive a packet before it expires.

2. We formulate an optimization problem to select the best helper nodes to perform CRB. With the accurate channel state information (CSI), the formulated optimization problem provides an upper bound of the CRB performance in maximizing the number of nodes with successful packet reception. The upper bound can be used as a benchmark for the performance evaluation of other helper selection schemes. 
3. We propose a channel prediction scheme to find the set of the best helper nodes to perform CRB. The proposed channel prediction uses the local information that are transmitted during the normal operation of D-TDMA to determine the channel quality, as well as node cardinality, to perform CRB.

Here, with a focus on developing a CRB scheme to improve transmission reliability of a broadcast service, we consider a network where all nodes have already reserved their time slots. The frame size is large such that there are no contending nodes seeking for time slots. Nodes are aware of positions and velocities of their OHS nodes, which can be shared periodically from the application layer of the protocol stack as some safety applications require such information for their normal operations [18].

\subsection{Node Cooperation for Broadcast Service in D- TDMA MAC}

In this section, we present a node cooperation based makeup framework, referred to as cooperative relay broadcasting, for D-TDMA based MAC protocols. One possible approach to implement a makeup strategy for broadcast service is by enabling node cooperation,

such that one-hop neighbors of a source node can help to rebroadcast a packet for safety applications. However, such a makeup transmission based on node cooperation should ensure that the required QoS be achieved. The requirement in the broadcast service gives rise to issues related to cooperation decisions, such as time slot selection and helper selection to perform CRB. Furthermore, extraction of node cardinality and channel quality information required for cooperation decisions adds challenges in developing the cooperative broadcast scheme. In the following, we discuss the cooperation decisions as a part of CRB framework in D-TDMA MAC to provide a reliable broadcast service in VANETs.

Consider nodes in an area of interest, e.g, within the one-hop transmission distance in either directions of a source node. When the source node, denoted as $S$, broadcasts a safety packet, referred to as tagged packet, to its one-hop neighboring nodes. Due to channel errors, some nodes fail to receive the tagged packet. Let the sets of nodes that have and do not have the tagged packet be denoted as $\mathcal{H}$ and $\mathcal{D}$, respectively. Nodes in sets $\mathcal{H}$ 
and $\mathcal{D}$ are referred to as potential helper nodes $(\mathrm{PHNs})$ and potential destination nodes (PDNs), respectively. Each tagged packet has an expiry time, after which the information in the tagged packet is not valid anymore. For presentation clarity, consider that a packet expires after the duration of one time frame from the start of source node's time slot. With cooperation enabled broadcast service, PHNs cooperate to rebroadcast the tagged packet to PDNs before the packet expires. Rebroadcasting of the tagged packet should not be done after its expiry time. The objective of CRB is to maximize the number of vehicles which successfully receive the tagged packet before the packet expires. Our strategy is that, in each available unreserved time slot, only one PHN performs CRB to serve PDNs that are in its one-hop transmission distance and are in a good channel condition. The CRB operations is detailed in the following:

1. Source node $S$ broadcasts a packet during its time slot to all its one-hop neighboring nodes, denoted by set $\mathcal{O}_{S}$. Without the loss of generality, we consider that the source node owns the first time slot.

2. Among all the nodes in $\mathcal{O}_{S}$, some nodes receive the packet and the rest fail to receive it; sets $\mathcal{H} \subseteq \mathcal{O}_{S}$ and $\mathcal{D} \subseteq \mathcal{O}_{S}$ denote the sets of such nodes, respectively.

3. As soon as the source node transmits the packet, PHNs in set $\mathcal{H}$ wait for the next immediate unreserved time slot to perform CRB of the packet to serve the PDNs in their corresponding OHSs.

4. During the next unreserved time slot, the best helper node $z \in \mathcal{H}$ performs CRB to make up for the transmission failures. The best helper node is the one which has a good channel condition to a maximum number of PDNs. How to choose the best helper node to rebroadcast the packet is to be discussed.

5. On receiving the packet successfully from the best helper nodes, a PDN may become a PHN, and sets $\mathcal{D}$ and $\mathcal{H}$ are updated accordingly.

6. Operations from step 4 are repeated, during the next unreserved time slot until the time frame finishes. 
The best helper node should be chosen in such a way that it improves the transmission reliability. The goals are to $(i)$ maximize the number of nodes which successfully receive the packet before it expires, and ( $i i)$ minimize the transmission delay, such that the broadcast packet can spread faster among one-hop neighbors of the source node. To achieve the goals, selection of the best helpers and unreserved time slots to perform CRB should be made based on information such as node cardinality, channel condition and time slot usage, without imposing extra overhead on the D-TDMA MAC.

In the following, we present helper selection for CRB of a tagged packet. First, we present an optimal helper selection scheme under the assumption of accurate CSI, in which each node is aware of channel conditions to its one-hop neighboring nodes. Such a helper selection scheme provides the maximum achievable performance gain over non-cooperative broadcasting and can be used to set a benchmark for performance evaluation. As the accurate knowledge of CSI requires a high overhead and therefore is not practical to implement, we further present a simple helper selection scheme based on local information, which is shown to provide a performance gain over existing D-TDMA MAC.

\subsection{Optimal Helper Selection with Accurate Channel Information}

We formulate an optimization problem to find the best helper node to perform CRB with precise CSI among nodes in the network. First, we describe the variables of the optimization problem and their relations with sets $\mathcal{D}$ and $\mathcal{H}$. Then, we formulate the optimization problem to select the best helper node for maximizing the number of nodes which successfully receive the tagged packet broadcast from the source node in a given unreserved time slot.

Define set $\mathcal{K}$ as

$$
\mathcal{K}=\left\{k_{x y} \mid k_{x y}=\{0,1\}, x \in \mathcal{D}, y \in \mathcal{H}\right\}
$$

where $k_{x y}$ is a binary indicator variable, equal to 1 if node $x$ in $\mathcal{D}$ receives the tagged packet from node $y$ in $\mathcal{H}$, and 0 otherwise. 
Similarly, define set $\mathcal{Q}$ as

$$
\mathcal{Q}=\left\{q_{y} \mid q_{y}=\{0,1\}, y \in \mathcal{H}\right\}
$$

where $q_{y}$ is a binary indicator variable, equal to 1 if node $y$ in $\mathcal{H}$ is selected to forward the packet, and 0 otherwise.

Let $\mathcal{V}$ be the set of transmission results which depend on channel quality, defined as

$$
\mathcal{V}=\left\{v_{y x} \mid v_{y x}=\{0,1\}, x, y \in\left\{\mathcal{O}_{s} \cup s\right\}\right\}
$$

where $v_{y x}$ is a binary indicator variable, equal to 1 if the transmission from node $y$ to node $x$ is successful and 0 otherwise.

Assume that set $\mathcal{V}$ is known to all the nodes in the area of interest. With the knowledge of set $\mathcal{V}$, we want to find a PHN in set $\mathcal{H}$ to relay the packet in a given unreserved time slot, such that the number of PDNs in $\mathcal{D}$ receiving the packet before it expires is maximized. Helper selection is required only if there is at least one PHN and one PDN, i.e., iif $|\mathcal{H}|>0$ and $|\mathcal{D}|>0$. The objective in the helper selection is to maximize $\sum_{x \in \mathcal{D}} \sum_{y \in \mathcal{H}} k_{x y}$. With the knowledge of set $\mathcal{V}$ and OHS information from exchanged FIs, sets $\mathcal{D}$ and $\mathcal{H}$ can be determined. There are constraints on $\mathcal{K}$ and $\mathcal{Q}$, as follows:

1. The cooperation opportunity during an unreserved time slot should be utilized. There must be at least one PHN to relay the tagged packet. On the other hand, in each unreserved time slot, there should be no more than one PHN to relay the packet, in order to avoid transmission collisions;

2. Each PDN should receive the packet relayed by only one PHN at a time. If two or more PHNs relay the packet to a PDN, collision occurs at the PDN, resulting in transmission failure and hence wasting the cooperation opportunity;

3. If node $y$ in $\mathcal{H}$ relays the packet, node $x$ in $\mathcal{D}$ receives the tagged packet only if $v_{y x}=1$.

Based on the preceding discussion, an optimization problem can be formulated for the helper selection and is given by 


$$
\begin{aligned}
\underset{k_{x y}, q_{y}}{\operatorname{maximize}} & \sum_{x \in \mathcal{D}} \sum_{y \in \mathcal{H}} k_{x y} & & \\
\text { Subject to: } & \sum_{y \in \mathcal{H}} q_{y}=1, & & \\
& \sum_{y \in \mathcal{H}} k_{x y} \leq 1, & & \forall x \in \mathcal{D}, \\
& k_{x y}-v_{y x} q_{y}=0, & & \forall y \in \mathcal{H}, \forall x \in \mathcal{D}, \\
& k_{x y}, q_{y}=\{0,1\} & & \forall y \in \mathcal{H}, \forall x \in \mathcal{D} .
\end{aligned}
$$

The optimization problem in (5.4) is a binary integer linear optimization problem and can be solved by using any binary integer linear programming technique. The best potential helper node, $y^{\prime}$, is the one among all the PHNs, which has $q_{y^{\prime}}=1$ from (5.4). Similarly, the set of optimum PDNs, denoted as $\mathcal{D}^{\prime}$, is a subset of $\mathcal{D}$ and is given by

$$
\mathcal{D}^{\prime}=\left\{x \mid x \in \mathcal{D}, v_{y^{\prime} x}=1\right\}
$$

After node $y^{\prime}$ rebroadcasts the packet, sets $\mathcal{D}$ and $\mathcal{H}$ are updated as

$$
\mathcal{D}=\mathcal{D}-\mathcal{D}^{\prime}, \quad \mathcal{H}=\mathcal{H} \cup \mathcal{D}^{\prime}
$$

\subsection{Helper Selection with Channel Prediction}

The optimal helper selection based on (5.4) requires the accurate knowledge of CSI, which is not practical to realize, especially in the highly dynamic vehicular networking environment. Here, a more realistic best helper selection scheme is presented. Each node in set $\mathcal{H}$ performs helper evaluation based on its local information, namely the number of OHS nodes, time slot usage information and its link quality. The first two information items can be extracted from FIs exchanged with its OHS nodes, while the link quality is estimated from positions and velocities of its OHS nodes.

A PHN, say $z \in \mathcal{H}$, considers itself as the best helper node to rebroadcast the tagged 
packet if it can successfully deliver the packet to a largest number of nodes that failed to receive the packet, referred to as failed nodes. While evaluating the number of neighboring failed nodes, node $z$ counts its one-hop neighbors that have already announced transmission failures during their time slots, referred to as reported failed nodes (whose time slots are earlier than the selected unreserved time slot in the current frame). Further, it predicts the transmission status of the remaining one-hop nodes that have not yet accessed the channel to send their FIs, referred to as predicted failed nodes. Let $\mathcal{R}_{z}^{f}$ and $\mathcal{P}_{z}^{f}$ denote the sets of reported and predicted fail nodes, respectively, from the perspective of node $z$. Hence, nodes in set $\mathcal{O}_{z}^{f}\left(\triangleq \mathcal{R}_{z}^{f} \cup \mathcal{P}_{z}^{f}\right)$ are the neighboring one-hop nodes which failed to receive the tagged packet. In addition, node $z$ determines the sets of reported and predicted successful nodes, denoted as $\mathcal{R}_{z}^{s}$ and $\mathcal{P}_{z}^{s}$ respectively, which have already announced and have not yet announced the successful reception of the tagged packet, respectively. Consequently, nodes in set $\mathcal{O}_{z}^{s}\left(\triangleq \mathcal{R}_{z}^{s} \cup \mathcal{P}_{z}^{s}\right)$ are the one-hop neighboring nodes which successfully received the tagged packet. Note that $\mathcal{R}_{z}^{s}$ and $\mathcal{R}_{z}^{f}$ are determined by node $z$ based on FIs that it received after the source node's time slot. On the other hand, node $z$ estimates $\mathcal{P}_{z}^{s}$ and $\mathcal{P}_{z}^{f}$. In order to avoid redundant transmissions, node $z$ considers CRBs that are already performed in its one-hop neighborhood while estimating the predicted sets. By doing so, it excludes nodes in $\mathcal{P}_{z}^{f}$ which may have received the packet during the previous CRBs. Next, we discuss how to determine the sets of predicted failed and successful nodes based on channel prediction.

\subsubsection{Prediction of Failed and Successful Nodes}

Channel quality is predicted based on a calculated average probability of successful communication, denoted as $\pi_{g}$. The channel quality is considered to be in a good condition, if the calculated probability value is greater or equal to a threshold value, denoted as $\pi_{t h}$. Hence, the channel is in a good condition if $\pi_{g} \geq \pi_{t h}$ and in a poor condition otherwise. The average probability of successful communication is calculated based on a two-state first-order Markov channel model (as discussed in Appendix B), such that $\pi_{g}$ is the steady state probability that the channel remains in the good state. As nodes are aware of the positions and velocities of their one-hop neighboring nodes, they can determine the probability of finding a channel in the good state, denoted as $\pi_{g}^{x y}$, from node $x$ to node $y$. Set 


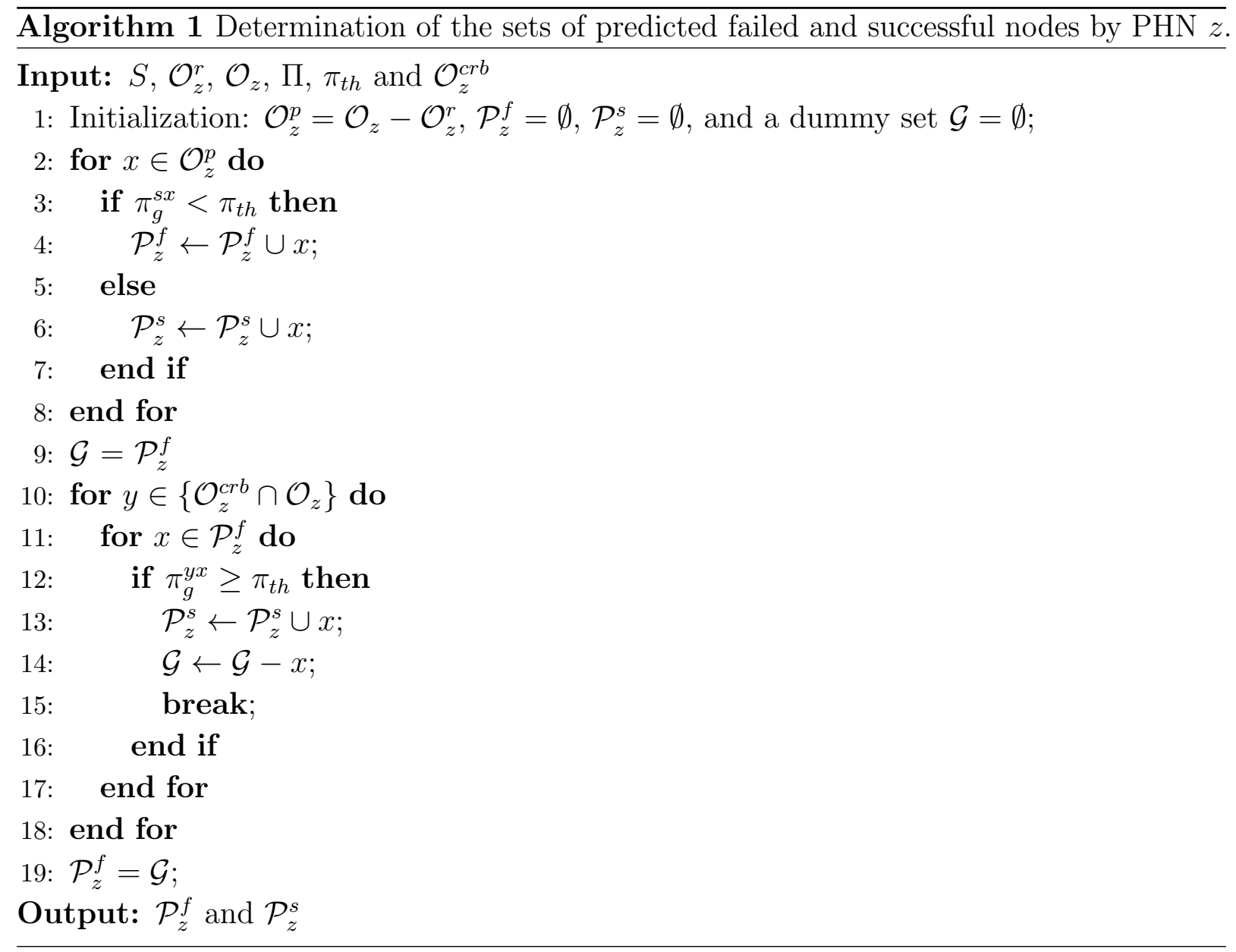

$\Pi=\left\{\pi_{g}^{x y}\right\}$ is then used to determine the sets of predicted failed and successful nodes. The procedure to determine such predicted sets is given in Algorithm 1 for node $z$, where $\mathcal{O}_{z}^{\text {crb }}$ denotes the set of PHNs that are in one-hop transmission distance from node $z$ and already performed CRB before the selected unreserved time slot, and $\mathcal{O}_{z}^{r}\left(\triangleq \mathcal{R}_{z}^{s} \cup \mathcal{R}_{z}^{f}\right)$ denotes the set of one-hop neighboring nodes of $z$ that have already announced their transmission status from the source node. 


\subsubsection{Cooperation Decisions}

Once $\mathcal{O}_{z}^{s}$ and $\mathcal{O}_{z}^{f}$ are determined, node $z$ evaluates itself and compares with successful nodes (both predicted and reported) in its one-hop neighborhood, i.e, nodes in set $\mathcal{O}_{z}^{s}$. First, it determines a set of failed nodes to which it can successfully relay the tagged packet in a given unreserved time slot, denoted as $\mathcal{A}_{z} \subseteq \mathcal{O}_{z}^{f}$. Then, it determines sets $\mathcal{A}_{y} \subseteq \mathcal{O}_{z}^{f}$ for node $y, \forall y \in \mathcal{O}_{z}^{s}$, as given in Algorithm 2. Based on $\mathcal{A}_{z}$ and $\mathcal{A}_{y}, \forall y \in \mathcal{O}_{z}^{s}$, node $z$ performs $\mathrm{CRB}$ in the selected unreserved time slot, if all of the following conditions are satisfied:

1. There is at least one failed node, either predicted or reported, which can successfully receive the tagged packet from $z$, i.e., $\left|\mathcal{A}_{z}\right|>0$;

2. Node $z$ can relay the packet to a largest number of failed nodes (both predicted and reported combined) among all nodes in $\mathcal{O}_{z}^{s}$, i.e., $\left|\mathcal{A}_{z}\right|>\left|\mathcal{A}_{y}\right|$ for any node $y$ in $\mathcal{O}_{z}^{s}$;

3. When there is one or more nodes that can relay to the same maximum number of failed nodes, node IDs will be used to make the cooperation decision. If $\left|\mathcal{A}_{z}\right|=\left|\mathcal{A}_{y}\right|$, $\forall y \in \mathcal{O}_{z}^{s}$, node $z$ relays the packet instead of node $y$ if node $z$ 's ID is less than the ID of node $y$.

\subsubsection{Cooperative Relay Broadcasting}

Errors may occur when a PHN predicts the channel conditions and, consequently, determines the sets of predicted failed and successful nodes. Due to such errors, two or more PHNs that are in each others' one-hop distance may find themselves as the best potential helper nodes to relay the tagged packet. In such an event, simultaneous CRBs result in transmission collisions and waste cooperation opportunities. To avoid such undesired events, a node uses an energy-burst or channel jamming signal, also known as black-burst [58]. In doing so, after finding itself as the best potential helper node, node $z$ transmits a black-burst for a random time interval, say $\delta_{z} \Delta$ time units from the start of a time slot, where $\delta_{z}$ is randomly drawn from set $\{1,2, \cdots, \delta\}$ and $\Delta$ is a fixed and small time duration (such as a slot time in the IEEE 802.11 based MAC protocols). Then, it listens to the channel and 


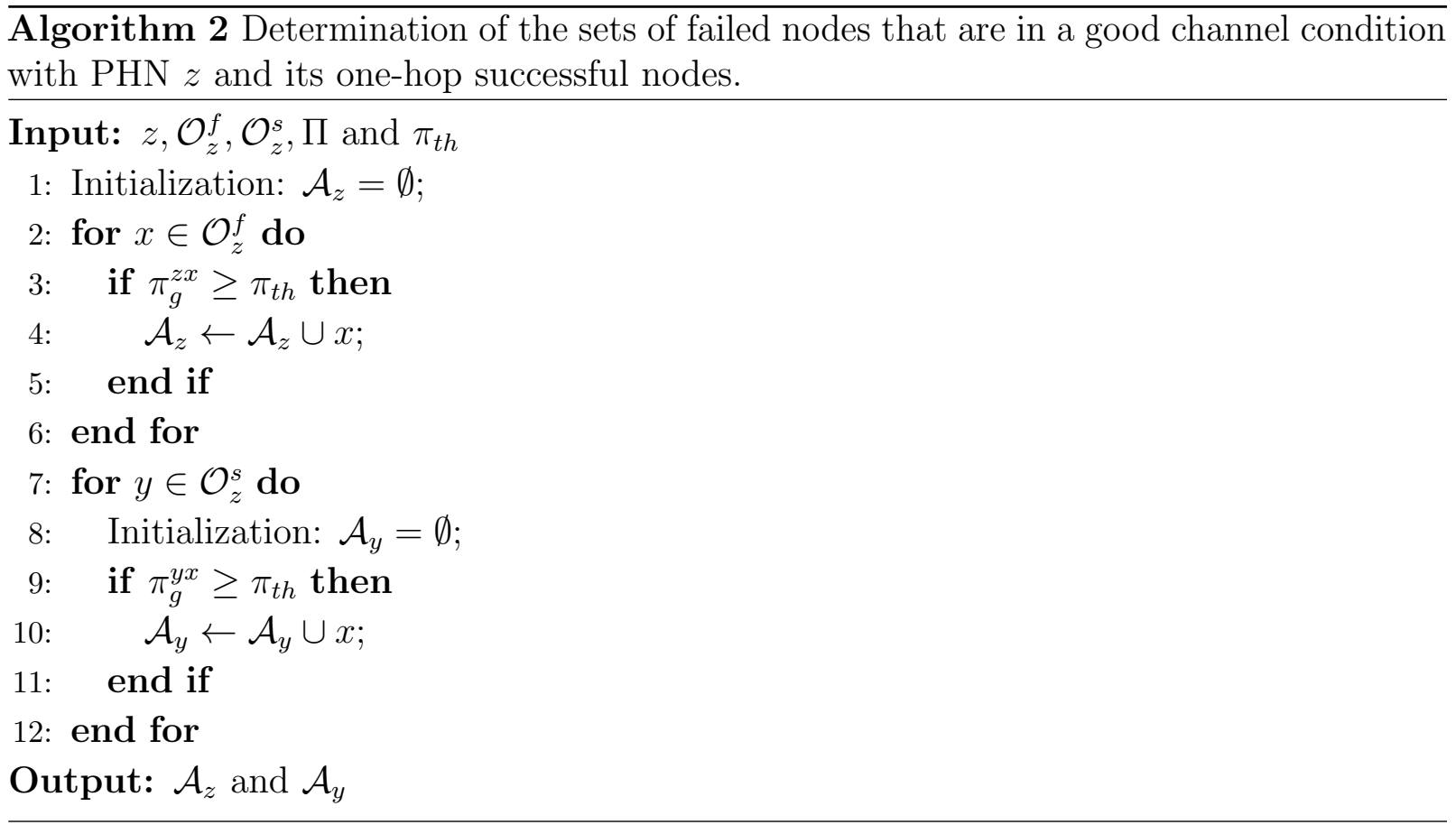

relays the tagged packet only if the channel is idle, as illustrated in Fig. 5.1. When node $y$ with a smaller black-burst period detects the black-burst from node $z$, it suspends its potential CRB. With a large $\delta$ value the probability that two or more PHNs choosing the same black-burst period is small. Thus, performing CRB after a random time interval from the start of a time slot reduces transmission collisions from two or more potential helper nodes in CRB. Note that the sum of $\delta \Delta$ time units and the transmission time of a CRB packet should be equal to the duration of a time slot. As each node owns a time slot to transmit a complete packet, repeated transmission of the FI during CRB is unnecessary. Hence, a packet from the best helper node consists of a packet header ( $\mathrm{PH})$, payload data and cyclic redundancy check (CRC). The absence of FI compensates for the black-burst period and should not affect the normal operation of D-TDMA.

Collisions occur only if two or more nodes that are not in each other's one-hop distance evaluate themselves as the best helper nodes. In such a case, they do not sense each others' black-burst signals and perform CRB, resulting in collisions at their common one-hop neighboring nodes. However, it matters only if the collisions occur at PDNs, as PHNs nodes 


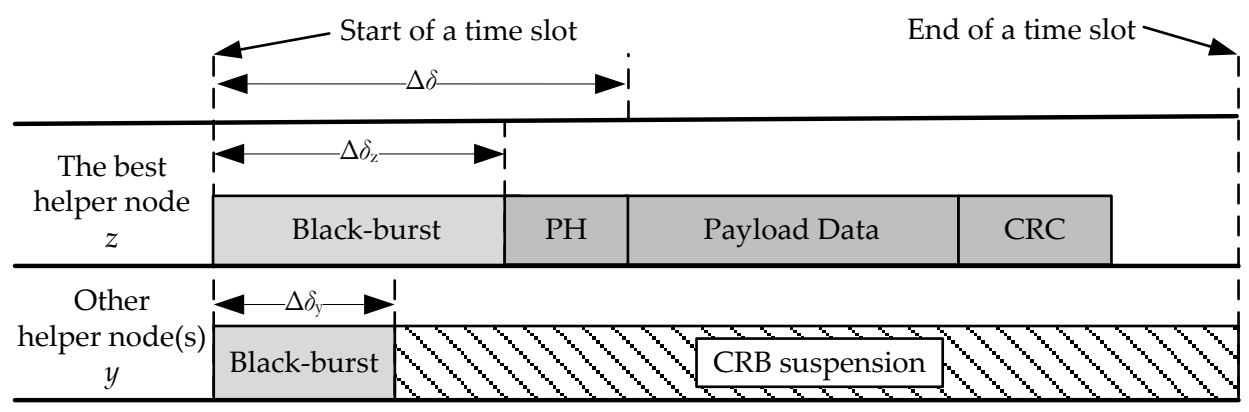

Figure 5.1: CRB performed by the best helper node and suspension of CRB(s) from the other potential helper node(s) that is (are) not within the one-hop distance from the transmitting helper node.

are not the target receivers during the CRB. Next, we present computer simulation results to evaluate the performance of the proposed CRB schemes.

\subsection{Simulation Results}

Simulations are performed in MATLAB considering the similar networking scenarios, road segment and node mobility as described in Section 4.4. As the channel estimation, as discussed in Appendix $\mathrm{B}$, is valid for integer $m$ values, here we consider $m=2$ for $R \leq 100$ meters. Performance of CRB schemes with optimal helper selection (CRB-OPT) and with helper selection based on channel prediction (CRB-HSCP) are evaluated and compared with the D-TDMA MAC, in terms of the transmission reliability and efficiency. The following performance metrics are considered:

1. Packet received rate, which is the ratio of the number of nodes in source node's one-hop neighborhood that received the packet, before it expires, to the total number of source node's one-hop neighbors [88]. It reflects the ability of a node to receive the tagged packet, either through direct transmission from a source node or through CRB, before it expires;

2. Packet delivery ratio, which is the ratio of the number of packets that are received by the required percentage of one-hop neighbors over the total number of broadcast 
packets [88]. It reflects the capability of a broadcast service to disseminate a packet to achieve the required QoS. Here, three QoS levels are considered: the tagged packet must be delivered to $50 \%, 75 \%$, and $99 \%$ of nodes in the one-hop neighborhood of the source node;

3. Normalized number of retransmission attempts, which is the ratio of the number of broadcast to the maximum number of possible broadcast of a packet in a frame to achieve the required QoS. It reflects the efficiency of a broadcast service to achieve the required QoS. Note that, for the system under consideration, a packet can be rebroadcast until there is no unreserved time slot before the packet expires. The maximum number of possible broadcast is the number of available unreserved time slots in a frame. Here, we consider a large frame size relative to the average number of THS nodes. Thus, from (3.6), the maximum number of possible broadcast is given by $F-N_{T}$.

Fig. 5.2 shows the packet received rate with different path-loss exponent values $(\alpha)$. With $\alpha=2$, the packet received rate of D-TDMA MAC reaches 1 , as a source node is capable of disseminating packets to all of its one-hop neighbors during its own time slot and does not require node cooperation to rebroadcast the packet. However, the packet received rate decreases to 0.88 and 0.29 , as the channel quality degrades for $\alpha=3$ and 4 respectively. At a moderate channel condition with $\alpha=3$, the CRB schemes recover the transmission failures from the source node and increase packet received rate to 1 . However with $\alpha=4$, the packet received rate of CRB schemes are lower than that with $\alpha=3$. The packet received rate reaches its peak when the number of nodes is moderate, relative to the number of time slots in a frame ${ }^{1}$. At a small or large $\bar{N}_{T}$ value, either there are no helper nodes to perform $\mathrm{CRB}$ or the number of PDNs is large and the number of unreserved time slots is not enough to serve all the PDNs, respectively. Hence, the packet received rate is lower as the $\alpha$ value increases from 3 to 4 .

Figs. 5.3 and 5.4 show the packet delivery ratio for different QoS requirements and with $\alpha$ equal to 3 and 4 , respectively. When the channel condition is good with $\alpha=2$,

\footnotetext{
${ }^{1}$ Here the average number of nodes per THS per frame, $\bar{N}_{T}$, is compared with the number of time slots per frame, $F$, such that $\bar{N}_{T} \ll F$ reflects a small number of nodes and $\bar{N}_{T} \approx F$ reflects a large number of nodes.
} 


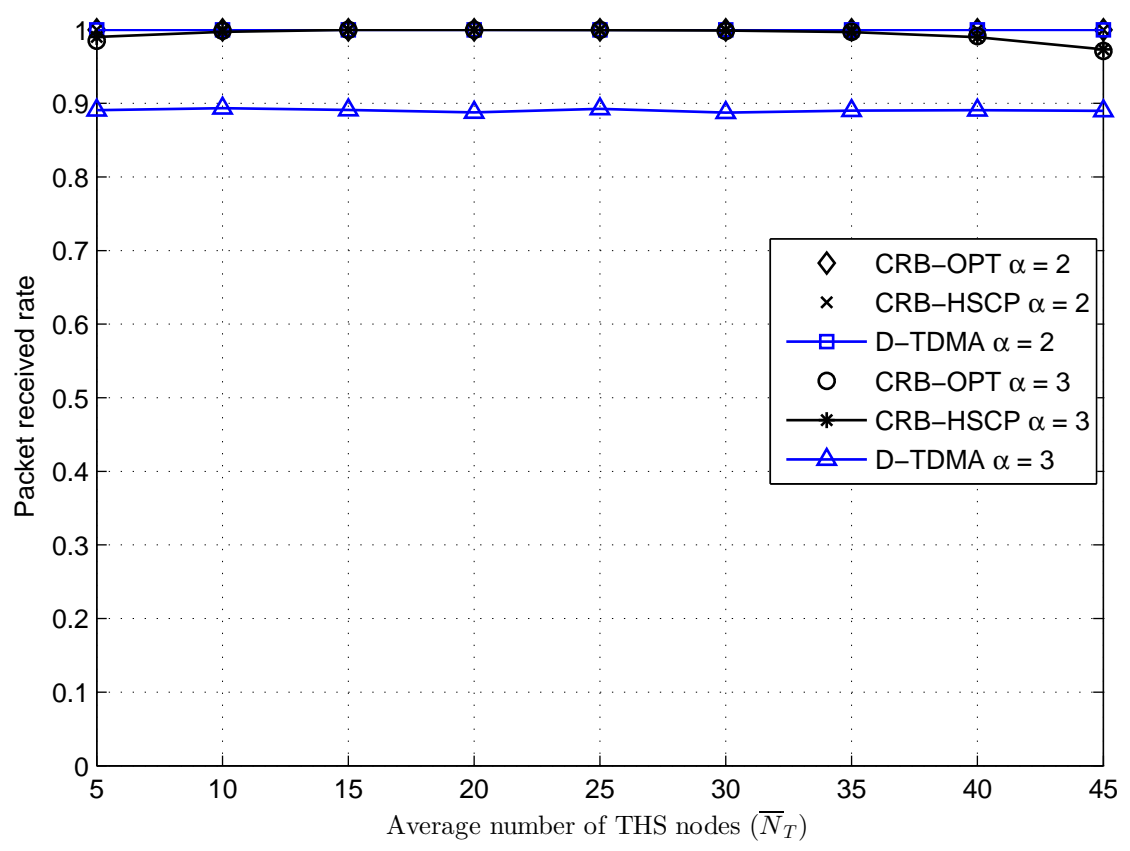

(a)

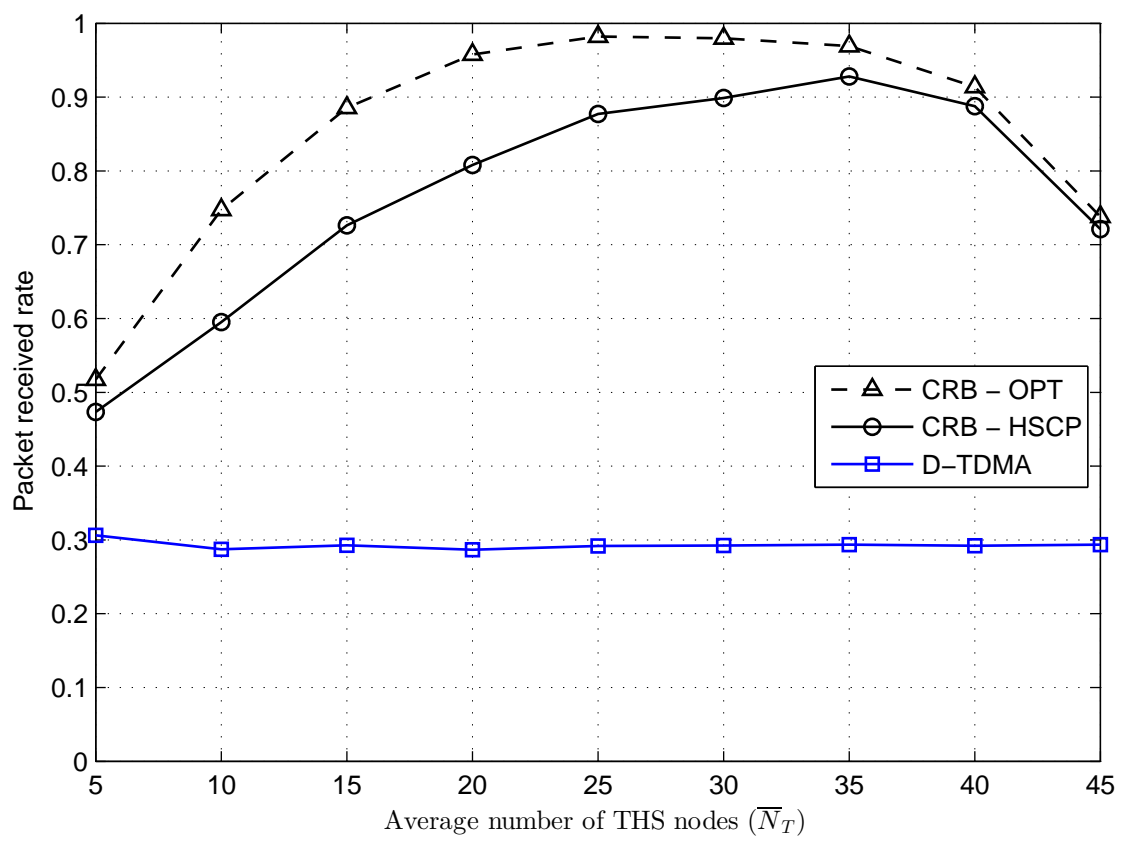

(b)

Figure 5.2: Packet received rate in D-TDMA MAC, CRB-OPT and CRB-HSCP with (a) $\alpha=2$ and 3 ; (b) $\alpha=4$. 


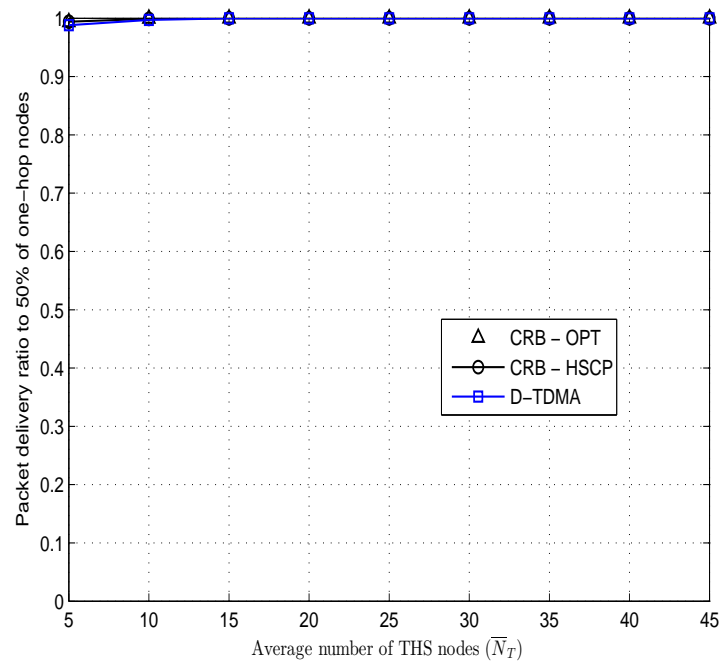

(a)

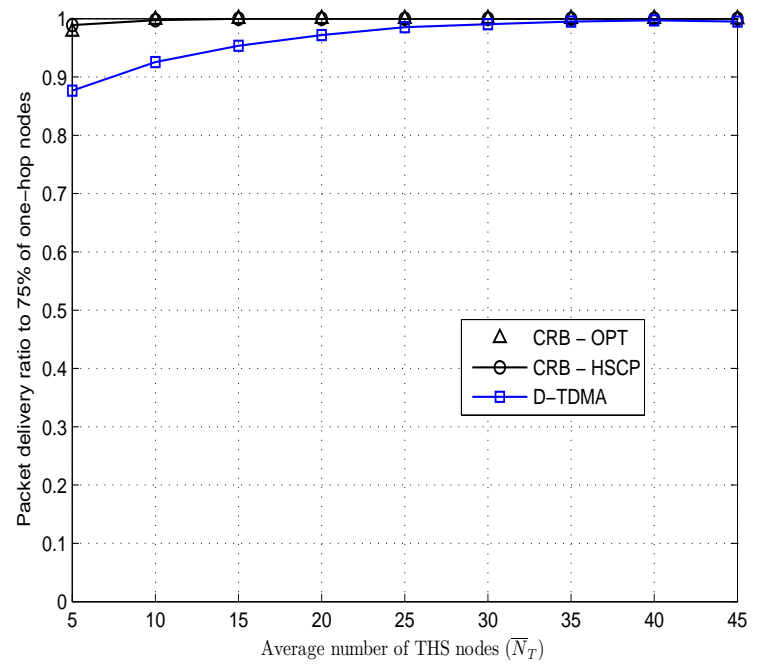

(b)

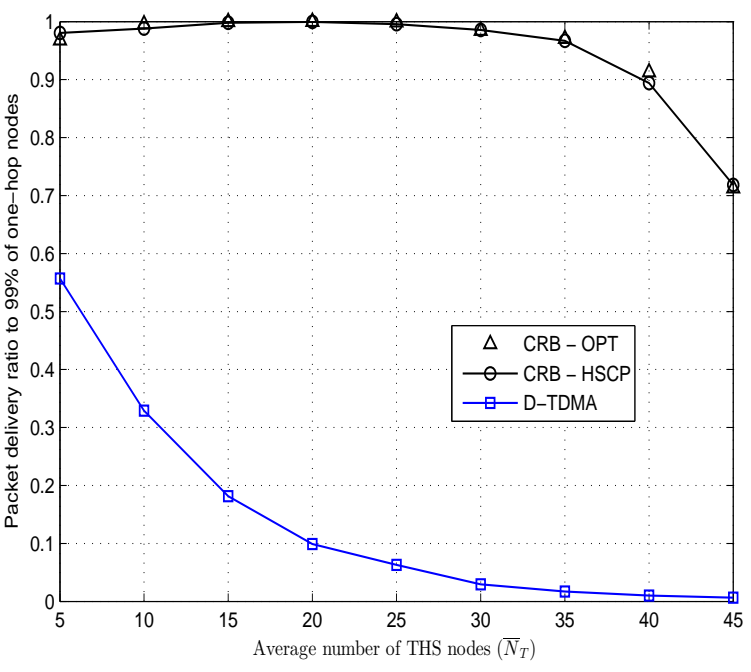

(c)

Figure 5.3: Packet delivery ratio with $\alpha=3$ in D-TDMA MAC, CRB-OPT and CRB-HSCP such that (a) $50 \%$; (b) $75 \%$; (c) $99 \%$ of nodes in the area of interest received packet within a duration of one time frame. 
D-TDMA MAC is capable of achieving a $99 \%$ delivery ratio. Thus, the packet delivery ratio with $\alpha=2$ are not included in the following analysis. As the channel degrades when $\alpha$ value increases to 3 and 4, D-TDMA fails to achieve the required QoS. Particularly, D-DTMA MAC can achieve only up to 75\% QoS level with $\alpha=3$, as in Fig. 5.3. Moreover, the packet delivery ratio decreases to 0 as the QoS requirement increases to $99 \%$, for a relatively high number of nodes. On the other hand, the packet delivery ratio reaches to 1 using CRB schemes with 99\% QoS requirement, even when the number of nodes increases. This is because the tagged packet is repeatedly rebroadcasted, such that all the one-hop neighbors of the source node receive the packet before it expires. When the channel quality degrades as $\alpha$ value increases to 4 , in Fig. 5.4, D-TDMA is not even effective to meet the $50 \%$ QoS requirement. With the CRB schemes, on the other hand, the packet delivery ratio improves when the average number of THS nodes is moderate. When the average number of THS nodes is relatively large or small, the packet delivery ratio decreases. Fig. 5.5 shows the normalized number of retransmission attempts of CRB schemes. When the number of nodes is relatively large and the channel is in a poor condition, the normalized number of retransmission attempts reaches 1 , because all the unreserved time slots are used for cooperative relay broadcasting to deliver the packet to a large number of failed nodes. On the other hand, when the number of nodes is relatively small, the normalized number of retransmission attempts is less than 0.1, due to the lack of PHNs to relay the tagged packet.

At a good $(\alpha=2)$ or moderate $(\alpha=3)$ channel condition, as in Figs. 5.2(a), 5.3 and 5.5(a), the CRB-HSCP scheme performs equally well in comparison with the CRB-OPT scheme. As the channel quality degrades with $\alpha=4$ as in Figs. 5.2(b) and 5.4, the channel prediction is not as effective as that of the earlier cases. Fig. 5.5(b) shows that the CRB-HSCP scheme performs more CRBs than the CRB-OPT scheme. This is mainly due to the errors in channel prediction and collisions among the best helper nodes that are not in each others' one-hop transmission distance, which are not considered in CRB-OPT. Hence, the performance of CRB-HSCP is lower than that of CRB-OPT when the channel is in a poor condition. However, the performance achieved by CRB-HSCP is significantly higher than that of D-TDMA MAC in all the cases, and even reaches up to that of CRB-OPT scheme, the upper limit, when the number of nodes is relatively large in the network. 


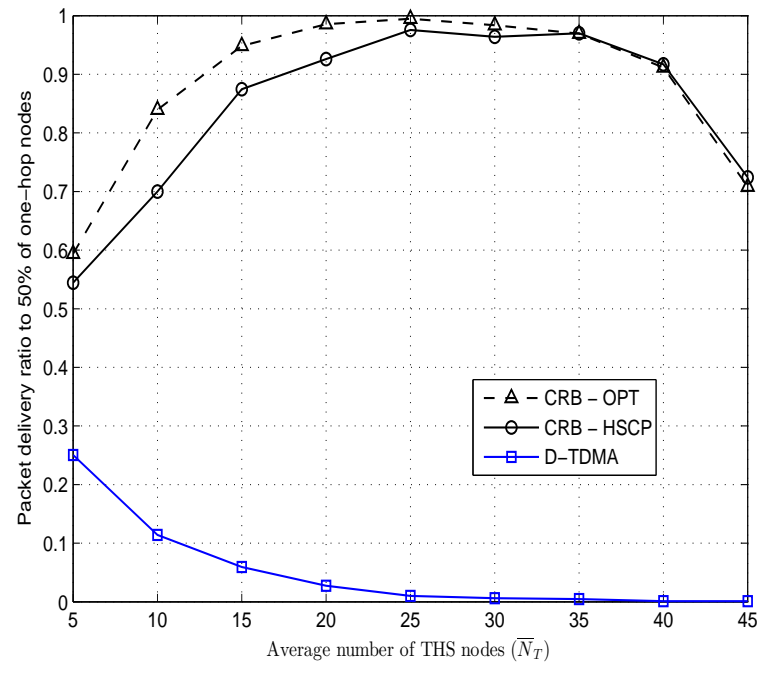

(a)

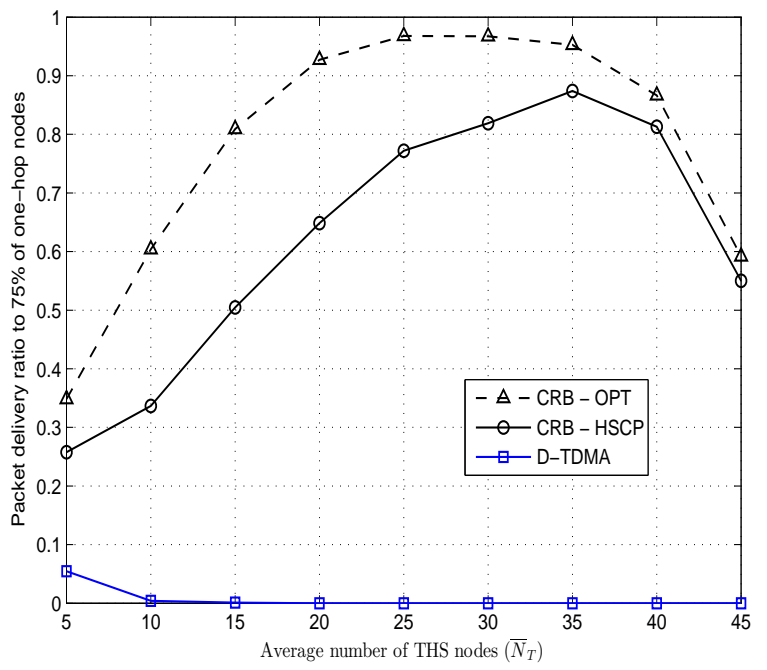

(b)

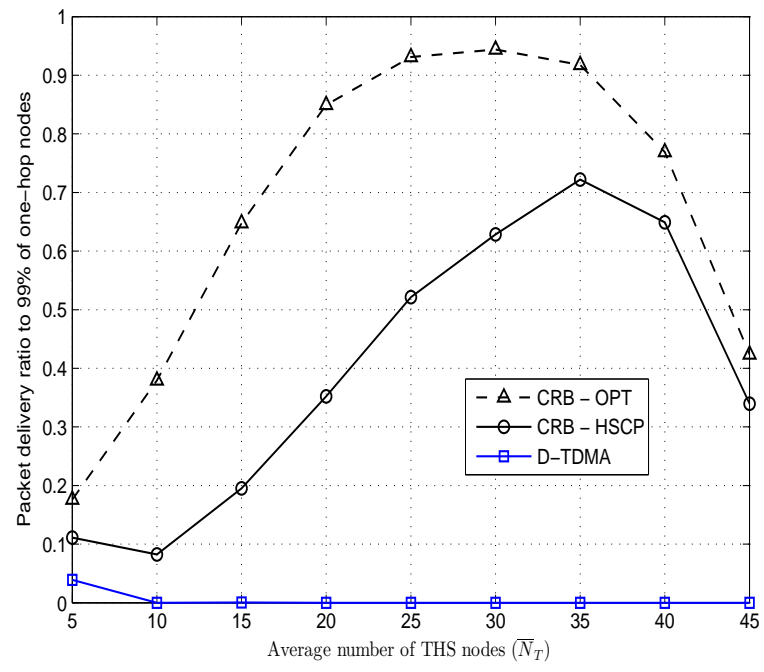

(c)

Figure 5.4: Packet delivery ratio with $\alpha=4$ in D-TDMA MAC, CRB-OPT and CRB-HSCP such that (a) 50\%; (b) 75\%; (c) 99\% of nodes in the area of interest received packet within a duration of one time frame. 


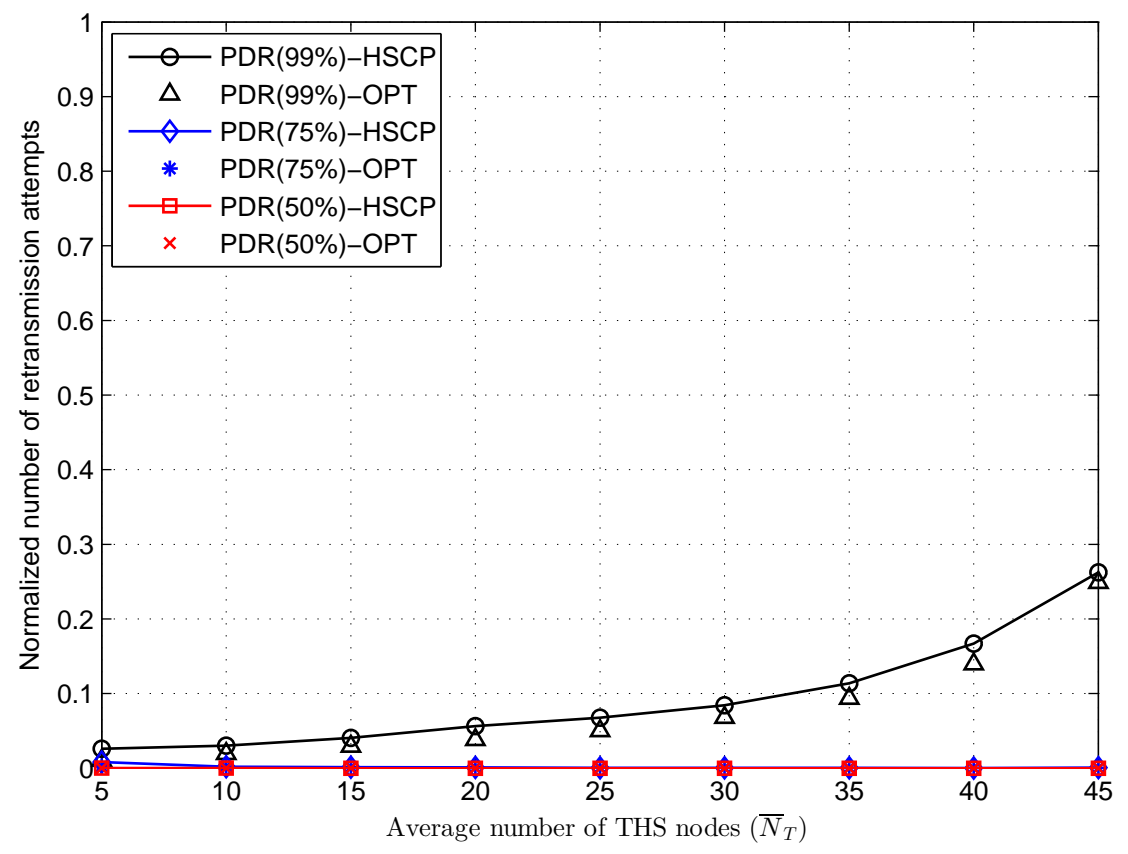

(a)

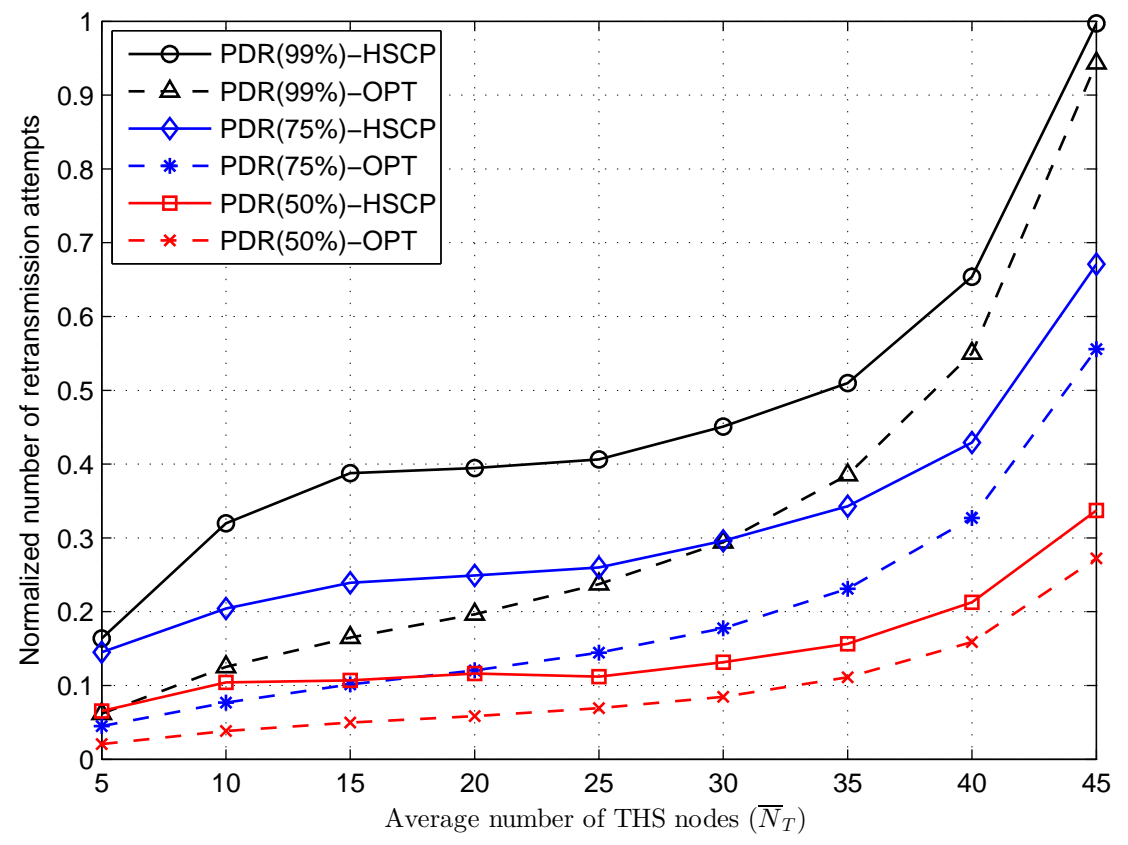

(b)

Figure 5.5: Normalized number of retransmission attempts over the number of unreserved time slot in CRB-OPT and CRB-HSCP with (a) $\alpha=3$; (b) $\alpha=4$. 


\subsection{Summary}

In this chapter, we present a node cooperation based makeup transmission framework for VANETs, referred to as cooperative relay broadcasting (CRB). The proposed CRB framework is a generic framework and can be implemented with any given helper selection scheme. To study the advantages of the proposed CRB framework, we first propose a helper selection scheme that requires a perfect knowledge of channel information which provides an upper bound of the CRB performance. Furthermore, we propose a realistic helper selection scheme that uses channel prediction based on the first-order two-state Markov channel, to evaluate helper nodes to perform CRB. Our analysis shows that the proposed CRB framework improves the reliability of the broadcast service in VANETs. 


\section{Chapter 6}

\section{Conclusions and Future Works}

\subsection{Conclusions}

Vehicular ad hoc networks will be an essential component of intelligent transportation systems, to enable a wide range of mobile distributed applications to improve the safety and efficiency of vehicle transportation and support onboard passenger infotainment. The existing MAC approaches for VANETs are not free from packet dropping and throughput reduction due to the dynamic networking conditions and are not always suitable to support the wide range of mobile distributed applications. On the other hand, link-layer node cooperation can be used to improve the performance, establishing a more efficient, robust and reliable communication among nodes. However, the existing link-layer node cooperation schemes are not suitable in the vehicular dynamic networking conditions and cannot be applied directly to VANETs. Thus, this thesis aims to present node cooperation frameworks at the MAC layer of VANETs to provide efficient and reliable communication to support the wide range of applications with strict service requirements, and to provide more robust communication to tackle the dynamic networking conditions.

We propose and analyze a cooperative MAC protocol, referred to as Cooperative ADHOC MAC, for distributed TDMA MAC in vehicular networks. In CAH-MAC, upon detecting a transmission failure between a pair of source and destination nodes, a neighboring node offers cooperation to relay the packet to the destination during an unreserved time slot. 
When the relative node mobility is negligible, numerical results show that the CAH-MAC performs better than the existing D-TDMA MAC under similar networking conditions. As unreserved time slots are used for retransmission, throughput improvement is achieved. In addition, as a packet is retransmitted by a helper node as oppose to the retransmission by the source node in the next frame, the transmission delay is reduced and packet dropping rate is decreased. Numerical results demonstrate that CAH-MAC performs better when the number of nodes in a two-hop neighborhood is moderate as compared with the total number of time slots available in a frame. Furthermore, performance gain is significant for a moderate channel condition.

In a dynamic networking environment, node cooperation during unreserved time slots may lead to conflicts in the form of cooperation collisions, thus disrupting the normal operations of the D-TDMA MAC. To tackle such conflicts, we present a collision avoidance scheme for the CAH-MAC protocol, referred to as enhanced Cooperative ADHOC MAC (eCAH-MAC). In eCAH-MAC, the cooperative relay transmission phase is delayed, so that cooperation collisions can be avoided. It uses available bandwidth resources efficiently in the presence of time slot reservation attempts, which is a consequence of vehicular network dynamics, improving the performance of node cooperation at the MAC layer protocol. Our analysis shows that effectiveness of node cooperation decreases with an increase in the number of nodes mainly due to increase in the number of reservation attempts. However, as time slot reservations are allowed despite of the scheduling of cooperative relay transmission, eCAH-MAC does not disrupt the normal operations of the D-TDMA MAC. Furthermore, we consider a practical channel model and vehicle traces to perform extensive simulations. We demonstrate the efficiency and robustness of eCAH-MAC in the presence dynamic networking environment. Through mathematical analysis and simulations, we observe that eCAH-MAC is capable of avoiding cooperation collisions by suspending a cooperative relay transmission phase, which allows more nodes, seeking time slots, to efficiently reserve unused time slots.

We present a node cooperation based makeup transmission framework for D-TDMA $\mathrm{MAC}$, referred to as cooperative relay broadcasting (CRB). In the proposed CRB scheme, nodes with a packet from a source node relay the packet until it expires, making it suitable for delay sensitive safety applications with strict QoS requirements. The packets are forwarded 
by the best helper nodes during unreserved time slots. Accordingly, we first propose an optimal helper selection scheme that requires accurate channel state information to select the best helper nodes. The proposed helper selection scheme provides an upper bound of the CRB performance, which can be used as a benchmark for performance evaluation. Furthermore, we propose a channel prediction based helper selection scheme that uses the local information to estimate the channel quality and selects the best helper nodes. Through extensive simulations, we observed that CRB is useful in a poor channel condition. Our analysis shows that the channel prediction based helper selection scheme performs equally well in comparison with the optimal helper selection scheme, when the channel condition and the number of nodes in the area of interest are moderate. However, as the channel quality degrades, due to errors in channel prediction and occurrence of transmission collisions, the performance of the proposed helper selection is lower than the maximum performance limit, but is significantly better than that of D-TDMA MAC.

\subsection{Future Research Directions}

The existing D-TDMA MAC protocols for VANETs do not comprise scheme(s) to handle an event when the number of nodes sharing a frame is too small or large as compared to the total number of time slots available in a frame. It is necessary to develop a scheme to handle such an issue to enhance the performance of D-TDMA MAC and make it more robust to tackle the change in vehicle density. One possible way to tackle such issue is by varying the transmission power $\left(P_{t}\right)$, which consequently varies the transmission range $(R)$ and adjusts the number of nodes in a given two-hop set sharing a frame. Hence, how to determine the occurrences of such undesired event and how to implement a scheme to resolve the event need further investigation. In addition, the existing D-TDMA MAC protocols suffer from the unnecessary loss of time slots due to a temporal channel fading. A node, after suffering from a transmission collision or channel error or both, releases its time slot to avoid conflicts. However, release of time slots due to channel errors may not be necessary and degrades the system performance. Hence, it is an important research topic to differentiate transmission collisions and channel errors in D-TDMA MAC, in order to avoid unnecessary loss of time slots and improve the system performance. Furthermore, to 
avoid negative impacts due to the unnecessary release of time slots, a maximum allowable transmission limit can be set, such that a node releases its time slot only if it does not detect its ID for a given number of consecutive frames due to poor channel conditions. With the aforementioned provisions, the proposed node cooperation schemes in this thesis are expected to be more effective in correcting the transmission failures due to a poor channel condition.

A more efficient helper selection scheme to perform CRB can be considered to evaluate the performance of the proposed CRB framework. Advance signal processing and/or network coding can be implemented to achieve an accurate channel estimation. Such accurate channel estimation will improve the performance of the proposed CRB framework, reduce the performance gap with the upper bound from optimal helper selection scheme, and result in a more efficient, reliable and robust broadcast service.

This thesis focuses on node cooperation for D-TDMA which operates in a single frequency channel. An investigation regarding the possibility of node cooperation for multi-channel MAC in VANETs is an interesting research direction. The existing D-TDMA multi-channel $\mathrm{MAC}$, such as VeMAC, is capable of providing a collision free broadcast service in the control channel $(\mathrm{CCH})$. A VeMAC-enabled node broadcasts packets belonging to safety applications in the $\mathrm{CCH}$ and gets ACKs (or NACKs) from all of its one-hop neighbors which own time slots. Also, it advertises its services, to be provided in a service channel (SCH), in the $\mathrm{CCH}$ (see Appendix A for detail). Provider and user exchange packets related to non-safety applications in the $\mathrm{SCH}(\mathrm{s})$, based on negotiations in the $\mathrm{CCH}$. However, channel coordination for VeMAC, to avoid a channel conflict problem, is not defined in [40]. A channel conflict arises due to the lack of channel coordination when two or more providers attempt to use the same service channel at the same time. When a new $s-d$ pair $^{1}$ agrees to use time slot(s) of an $\mathrm{SCH}$, which was already agreed on by a different $s-d$ pair, a channel conflict problem occurs. This is due to the lack of coordination among nodes (such as exchanging channel usage information) and relative mobility among the nodes. In VeMAC, when channel conflicts occur, the provider (which first reserves the channels) aborts the agreed negotiation, to avoid the possible collisions, and the new $s-d$ pair gets the channel access. Hence, how to implement a node cooperation approach, for instance

\footnotetext{
${ }^{1} \mathrm{~A}$ pair of provider and user is denoted as an $s-d$ pair.
} 
deploying cooperation among one-hop nodes to exchange the channel usage information, to solve the channel conflict problem needs further research. 


\section{References}

[1] L. Cheng, B. Henty, F. Bai, and D. Stancil, "Doppler spread and coherence time of rural and highway vehicle-to-vehicle channels at $5.9 \mathrm{GHz}$," in Proc. IEEE Globecom, Nov. 2008.

[2] United States Department of Transportation, "Intelligent transportation systems." [Online]. Available: http://www.its.dot.gov/index.htm

[3] http://goo.gl/Oe1hBG.

[4] R. Baldessari, B. Bdekker, A. Brakemeier, M. Deegener, A. Festag, W. Franz, A. Hiller, C. Kellum, T. Kosch, A. Kovacs, M. Lenardi, A. Lbke, C. Menig, T. Peichl, M. Roeckl, D. Seeberger, M. Strassberger, H. Stratil, H.-J. Vgel, Weyl, and W. Zhang, "Car-2-car communication consortium manifesto," Tech. Rep. Version 1.1, Aug. 2007.

[5] "FleetNet Projects." [Online]. Available: http://uk.nec.com/en_GB/emea/about/ neclab_eu/projects/fleetnet.html

[6] "CarTalk Projects." [Online]. Available: http://www.cartalk2000.net/

[7] "Network on Wheels Projects." [Online]. Available: http://www.network-on-wheels.de/

[8] "SAFESPOT Projects." [Online]. Available: http://www.safespot-eu.org/

[9] "CVIS Projects." [Online]. Available: http://www.cvisproject.org/

[10] "Coopers Projects." [Online]. Available: http://www.coopers-ip.eu/ 
[11] "Car-to-Car Communication Consortium." [Online]. Available: http://www.car-2-car. org/

[12] "Vehicle Infrastructure Integration." [Online]. Available: http://www. vehicle-infrastructure.org/

[13] J. Jakubiak and Y. Koucheryavy, "State of the art and research challenges for VANETs," in Proc. $5^{\text {th }}$ IEEE Consumer Communications and Networking Conference, Jan. 2008.

[14] H. Moustafa and Y. Zhang, Vehicular Networks: Techniques, Standards, and Applications. Boston, MA, USA: Auerbach Publications, 2009.

[15] H. Hartenstein and K. Laberteaux, "A tutorial survey on vehicular ad hoc networks," IEEE Commun. Mag., vol. 46, no. 6, pp. 164-171, June 2008.

[16] E. Schoch, F. Kargl, M. Weber, and T. Leinmuller, "Communication patterns in VANETs," IEEE Commun. Mag., vol. 46, no. 11, pp. 119 -125, Nov. 2008.

[17] G. A. G. Mosqueda, R. A. Santos, L. A. V. Gonzlez, V. R. Licea, and A. E. Block, Mobile Ad-Hoc Networks: Applications, $1^{\text {st }}$ ed. Rijeka, Croatia: Janeza Trdine, 2011.

[18] The CAMP Vehicle Safety Communications Consortium, Tech. Rep. DOT HS 809 859, "Vehicle safety communications project task 3 final report," Mar. 2005.

[19] H. T. Cheng, H. Shan, and W. Zhuang, "Infotainment and road safety service support in vehicular networking: From a communication perspective," Mech. Syst. Signal Process., vol. 25, no. 6, pp. 2020-2038, 2011.

[20] F. Bai, D. D. Stancil, and H. Krishnan, "Toward understanding characteristics of dedicated short range communications (DSRC) from a perspective of vehicular network engineers," in Proc. Int. Conf. Mobile Compt. and Netw., 2010.

[21] C. Mecklenbrauker, A. Molisch, J. Karedal, F. Tufvesson, A. Paier, L. Bernado, T. Zemen, O. Klemp, and N. Czink, "Vehicular channel characterization and its implications for wireless system design and performance," Proceedings of the IEEE, vol. 99, no. 7, pp. 1189-1212, July 2011. 
[22] J. Karedal, N. Czink, A. Paier, F. Tufvesson, and A. Molisch, "Path loss modeling for vehicle-to-vehicle communications," IEEE Trans. Veh. Technol., vol. 60, no. 1, pp. 323-328, Jan. 2011.

[23] Q. Xu, T. Mak, J. Ko, and R. Sengupta, "Vehicle-to-vehicle safety messaging in DSRC," in Proc. 1st ACM Int. Workshop Veh. Ad Hoc Networks, 2004.

[24] X. Ma, J. Zhang, X. Yin, and K. Trivedi, "Design and analysis of a robust broadcast scheme for VANET safety-related services," IEEE Trans. Veh. Technol., vol. 61, no. 1, pp. 46-61, Jan. 2012.

[25] F. Yu and S. Biswas, "Self-configuring TDMA protocols for enhancing vehicle safety with DSRC based vehicle-to-vehicle communications," IEEE J. Sel. Areas Commun., vol. 25 , no. 8, Oct. 2007.

[26] F. Ros, P. Ruiz, and I. Stojmenovic, "Acknowledgment-based broadcast protocol for reliable and efficient data dissemination in vehicular ad hoc networks," IEEE Trans. Mobile Comput., vol. 11, no. 1, pp. 33-46, Jan. 2012.

[27] F. Watanabe, M. Fujii, M. Itami, and K. Itoh, "An analysis of incident information transmission performance using MCS/CDMA scheme," in Proc. IEEE Intell. Veh. Symp., June 2005.

[28] H. Nakata, T. Inoue, M. Itami, and K. Itoh, "A study of inter vehicle communication scheme allocating PN codes to the location on the road," in Proc. IEEE Intell. Transp. Syst., Oct. 2003, pp. 1527-1532.

[29] J. J. Blum and A. Eskandarian, "A reliable link-layer protocol for robust and scalable intervehicle communications," IEEE Trans. Intell. Transp. Syst., vol. 8, no. 1, pp. 4-13, Mar. 2007.

[30] R. Mangharam, R. Rajkumar, M. Hamilton, P. Mudalige, and F. Bai, "Boundedlatency alerts in vehicular networks," in Proc. Mobile Netw. Veh. Environments, May 2007. 
[31] B. Sikdar, "Design and analysis of a MAC protocol for vehicle to roadside networks," in Proc. IEEE WCNC, Apr. 2008, pp. 1691-1696.

[32] "IEEE standard for information technology-local and metropolitan area networksspecific requirements-Part 11: Wireless LAN medium access control (MAC) and physical layer (PHY) specifications Amendment 6: Wireless access in vehicular environments," IEEE Std 802.11p-2010 (Amendment to IEEE Std 802.11-2007 as amended by IEEE Std 802.11k-2008, IEEE Std 802.11r-2008, IEEE Std 802.11y-2008, IEEE Std 802.11n-2009, and IEEE Std 802.11w-2009), pp. 1-51, July 2010.

[33] S. Eichler, "Performance evaluation of the IEEE 802.11p WAVE communication standard," in Proc. IEEE VTC Fall, Oct. 2007.

[34] Y. Wang and B. Bensaou, "Achieving fairness in IEEE 802.11 DFWMAC with variable packet lengths," in Proc. IEEE Globecom, 2001.

[35] F. Borgonovo, A. Capone, M. Cesana, and L. Fratta, "ADHOC MAC: New MAC architecture for ad hoc networks providing efficient and reliable point-to-point and broadcast services," Wireless Networks, vol. 10, pp. 359-366, 2004.

[36] M. Hassan, H. Vu, and T. Sakurai, "Performance analysis of the IEEE 802.11 MAC protocol for DSRC safety applications," IEEE Trans. Veh. Technol., vol. 60, no. 8, Oct. 2011.

[37] D. Jiang, V. Taliwal, A. Meier, W. Holfelder, and R. Herrtwich, "Design of 5.9 GHz DSRC-based vehicular safety communication," IEEE Wireless Commun., vol. 13, no. 5, pp. 36-43, Oct. 2006.

[38] F. Borgonovo, L. Campelli, M. Cesana, and L. Coletti, "MAC for ad-hoc inter-vehicle network: Services and performance," in Proc. IEE VTC Fall, Oct. 2003.

[39] F. Borgonovo, L. Campelli, M. Cesana, and L. Fratta, "Impact of user mobility on the broadcast service efficiency of the ADHOC MAC protocol," in Proc. IEEE VTC Spring, June 2005. 
[40] H. Omar, W. Zhuang, and L. Li, "VeMAC: A TDMA-based MAC protocol for reliable broadcast in VANETs," IEEE Trans. Mobile Comput., vol. 12, no. 9, pp. 1724-1736, Sept. 2013.

[41] H. Omar, W. Zhuang, A. Abdrabou, and L. Li, "Performance evaluation of VeMAC supporting safety applications in vehicular networks," IEEE Trans. Emerg. Topics Comput., vol. 1, no. 1, pp. 69-83, June 2013.

[42] H. Zhu and G. Cao, "rDCF: A relay-enabled medium access control protocol for wireless ad hoc networks," IEEE Trans. Mobile Comput., vol. 5, no. 9, pp. 1201-1214, Sept. 2006.

[43] P. Liu, Z. Tao, S. Narayanan, T. Korakis, and S. S. Panwar, "CoopMAC: A cooperative MAC for wireless LANs," IEEE J. Sel. Areas Commun., vol. 25, no. 2, pp. 340-354, Feb. 2007.

[44] S. Moh and C. Yu, "A cooperative diversity-based robust MAC protocol in wireless ad hoc networks," IEEE Trans. Parallel Distrib. Syst., vol. 22, no. 3, pp. 353-363, Mar. 2011.

[45] Z. Hu and C.-K. Tham, "CC-MAC: Coordinated cooperative MAC for wireless LANs," Comput. Networks, vol. 54, no. 4, pp. 618-630, 2010.

[46] H. Shan, W. Zhuang, and Z. Wang, "Distributed cooperative MAC for multihop wireless networks," IEEE Commun. Mag., vol. 47, no. 2, pp. 126-133, Feb. 2009.

[47] J. Zhang, Q. Zhang, and W. Jia, "VC-MAC: A cooperative MAC protocol in vehicular networks," IEEE Trans. Veh. Technol., vol. 58, no. 3, pp. 1561-1571, Mar. 2009.

[48] T. Zhou, H. Sharif, M. Hempel, P. Mahasukhon, W. Wang, and T. Ma, "A novel adaptive distributed cooperative relaying MAC protocol for vehicular networks," IEEE J. Sel. Areas Commun., vol. 29, no. 1, pp. 72-82, Jan. 2011.

[49] H. Shan, H. T. Cheng, and W. Zhuang, "Cross-layer cooperative MAC protocol in distributed wireless networks," IEEE Trans. Wireless Commun., vol. 10, no. 8, pp. 2603-2615, Aug. 2011. 
[50] A. Sadek, K. Liu, and A. Ephremides, "Collaborative multiple-access protocols for wireless networks," in Proc. IEEE ICC, June 2006.

[51] G. Yuan, M. Peng, and W. Wang, "Opportunistic user cooperative relaying in TDMAbased wireless networks," Wireless Commun. and Mobile Comput., vol. 10, no. 7, pp. 972-985, July 2010.

[52] Z. Yang, Y.-D. Yao, X. Li, and D. Zheng, "A TDMA-based MAC protocol with cooperative diversity," IEEE Commun. Lett., vol. 14, no. 6, pp. 542-544, June 2010.

[53] J.-K. Lee, H.-J. Noh, and J. Lim, "Dynamic cooperative retransmission scheme for TDMA systems," IEEE Commun. Lett., vol. 16, no. 12, pp. 2000-2003, Dec. 2012.

[54] X. Liu, C. Chen, A. Huang, and Q. Zhou, "A new TDMA-based cooperative MAC scheme," in Proc. Int. Conf. Telecomm., Apr. 2015.

[55] J.-K. Lee, H.-J. Noh, and J. Lim, "TDMA-based cooperative MAC protocol for multi-hop relaying networks," IEEE Commun. Lett., vol. 18, no. 3, pp. 435-438, Mar. 2014.

[56] M. Li, K. Zeng, and W. Lou, "Opportunistic broadcast of event-driven warning messages in vehicular ad hoc networks with lossy links," Comput. Networks, vol. 55, no. 10, pp. 2443-2464, 2011.

[57] Z. J. Haas, J. Y. Halpern, and L. Li, "Gossip-based ad hoc routing," IEEE/ACM Trans. Netw., vol. 14, no. 3, pp. 479-491, June 2006.

[58] G. Korkmaz, E. Ekici, and F. Ozguner, "Black-burst-based multihop broadcast protocols for vehicular networks," IEEE Trans. Veh. Technol., vol. 56, no. 5, pp. 3159-3167, Sept. 2007.

[59] N. Wisitpongphan, O. Tonguz, J. Parikh, P. Mudalige, F. Bai, and V. Sadekar, "Broadcast storm mitigation techniques in vehicular ad hoc networks," IEEE Wireless Commun., vol. 14, no. 6, pp. 84-94, Dec. 2007.

[60] S. Bharati and W. Zhuang, "Performance analysis of cooperative ADHOC MAC for vehicular networks," in Proc. IEEE Globecom, Dec. 2012. 
[61] S. Bharati and W. Zhuang, "CAH-MAC: Cooperative ADHOC MAC for vehicular networks," IEEE J. Sel. Areas Commun., vol. 31, no. 9, pp. 470-479, Sept. 2013.

[62] S. Bharati, L. Thanayankizil, F. Bai, and W. Zhuang, "Effects of time slot reservation in cooperative ADHOC MAC for vehicular networks," in Proc. IEEE ICC, June 2013.

[63] S. Bharati, W. Zhuang, L. Thanayankizil, and F. Bai, "Link-layer cooperation based on distributed TDMA MAC for vehicular networks," IEEE Trans. Veh. Technol. (submitted).

[64] S. Bharati and W. Zhuang, "CRB: A cooperative relay broadcasting framework to support safety applications in vehicular networks," IEEE Trans. Veh. Technol. (submitted).

[65] W. Ding, J. Wang, Y. Li, P. Mumford, and C. Rizos, "Time synchronization error and calibration in integrated GPS/INS systems," ETRI J., vol. 30, no. 1, pp. 59-67, Feb. 2008.

[66] P. Mumford, "Relative timing characteristics of the one pulse per second (1PPS) output pulse of three GPS receivers," in Proc. 6th Int. Symp. Satellite Navigation Technol. Including Mobile Positioning and Location Services, July 2003.

[67] W. Franz, H. Hartenstein, and M. Mauve, Inter-vehicle communications based on ad hoc networking principles: the FleetNet project. Universitätsverlag Karlsruhe, 2005.

[68] J. Laneman, D. Tse, and G. Wornell, "Cooperative diversity in wireless networks: Efficient protocols and outage behavior," IEEE Trans. Inf. Theory, vol. 50, no. 12, pp. 3062-3080, Dec. 2004.

[69] A. D. May, Traffic Flow Fundamentals, $1^{\text {st }}$ ed. Prentice Hall, 1990.

[70] H. Reijmers and R. Prasad, "The influence of vehicle distribution models on packet success probability on a three lane motorway," in Proc. IEEE VTC, May 1998.

[71] R. Fantacci and S. Nannicini, "Multiple access protocol for integration of variable bit rate multimedia traffic in UMTS/IMT-2000 based on wideband CDMA," IEEE J. Sel. Areas Commun., vol. 18, no. 8, pp. 1441-1454, Aug. 2000. 
[72] D. Vassis and G. Kormentzas, "Delay performance analysis and evaluation of IEEE 802.11e EDCA in finite load conditions," Wirel. Pers. Commun., vol. 34, no. 1-2, pp. 29-43, July 2005.

[73] http://vision-traffic.ptvgroup.com/en-us/products/ptv-vissim/.

[74] L. Cheng, B. Henty, D. Stancil, F. Bai, and P. Mudalige, "Mobile vehicle-to-vehicle narrow-band channel measurement and characterization of the $5.9 \mathrm{GHz}$ dedicated short range communication (DSRC) frequency band," IEEE J. Sel. Areas Commun., vol. 25, no. 8, pp. 1501-1516, Oct. 2007.

[75] M. K. Simon and M.-S. Alouini, Digital Communication over Fading Channels, $2^{\text {nd }}$ Edition. Wiley-IEEE Press, 2005.

[76] M. Torrent-Moreno, S. Corroy, F. Schmidt-Eisenlohr, and H. Hartenstein, "IEEE 802.11-based one-hop broadcast communications: Understanding transmission success and failure under different radio propagation environments," in Proc. ACM Int. Symp. MSWiM, 2006.

[77] G. M. T. Abdalla, M. A. Abu-Rgheff, and S.-M. Senouci, "An adaptive channel model for VBLAST in vehicular networks," EURASIP J. Wireless Commun. and Netw., vol. 11, 2009.

[78] W. C. Jakes and D. C. Cox, Eds., Microwave Mobile Communications. Wiley-IEEE Press, 1994.

[79] S. Lin, Y. Li, Y. Li, B. Ai, and Z. Zhong, "Finite-state Markov channel modeling for vehicle-to-infrastructure communications," in Proc. IEEE WiVeC, Sept. 2014.

[80] F. Lopez-Martinez, D. Morales-Jimenez, E. Martos-Naya, and J. Paris, "On the bivariate Nakagami-m cumulative distribution function: Closed-form expression and applications," IEEE Trans. Commun., vol. 61, no. 4, pp. 1404-1414, Apr. 2013.

[81] C. Pimentel, T. Falk, and L. Lisboa, "Finite-state Markov modeling of correlated Rician-fading channels," IEEE Trans. Veh. Technol., vol. 53, no. 5, pp. 1491-1501, Sept. 2004. 
[82] A. Borhani and M. Patzold, "Correlation and spectral properties of vehicle-to-vehicle channels in the presence of moving scatterers," IEEE Trans. Veh. Technol., vol. 62, no. 9, pp. 4228-4239, Nov. 2013.

[83] M. Rubinstein, I. Moraes, M. Campista, L. Costa, and O. Duarte, "A survey on wireless ad hoc networks," Mobile and Wireless Commun. Networks, vol. 211, pp. 1-33, Jan. 2006.

[84] N. Wisitpongphan, F. Bai, P. Mudalige, V. Sadekar, and O. Tonguz, "Routing in sparse vehicular ad hoc wireless networks," IEEE J. Sel. Areas Commun., vol. 25, no. 8, pp. 1538-1556, Oct. 2007.

[85] R. Wiedemann, Simulation des Strassenverkehrsflusses. Band 8, Karlsruhe, Germany: Schriftenreihe des Instituts fr Verkehrswesen der Universitt Karlsruhe, 1974.

[86] J. Filho, M. Yacoub, and G. Fraidenraich, "A simple accurate method for generating autocorrelated Nakagami-m envelope sequences," IEEE Commun. Lett., vol. 11, no. 3, pp. 231-233, Mar. 2007.

[87] M. Boban, T. Vinhoza, M. Ferreira, J. Barros, and O. Tonguz, "Impact of vehicles as obstacles in vehicular ad hoc networks," IEEE J. Sel. Areas Commun., vol. 29, no. 1, pp. 15-28, Jan. 2011.

[88] X. Ma, J. Zhang, and T. Wu, "Reliability analysis of one-hop safety-critical broadcast services in VANETs," IEEE Trans. Veh. Technol., vol. 60, no. 8, pp. 3933-3946, Oct. 2011.

[89] "IEEE standard for wireless access in vehicular environments (wave)-Multi-channel operation," IEEE Std 1609.4-2010 (Revision of IEEE Std 1609.4-2006), pp. 1-89, 2011.

[90] C. Campolo and A. Molinaro, "Cooperative multichannel management in IEEE 802.11p/WAVE vehicular ad hoc networks," Int. J. Veh. Inf. and Commun. Syst., vol. 2, no. 3/4, pp. 147-176, 2011. 
[91] M. Nakagami, "The m-distribution - A general formula of intensity distribution of rapid fading," Statistical Methods Radio Wave Propag., 1960. 


\section{Appendix A}

\section{Multi-Channel Operation in VANETs}

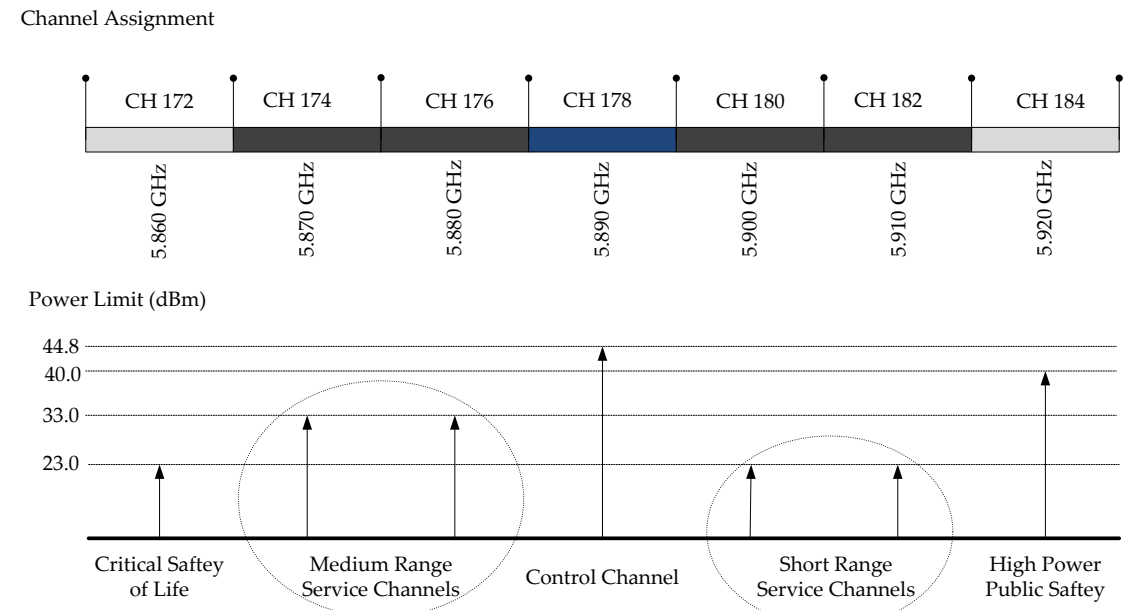

Figure A.1: Spectrum and Power Allocation for DSRC.

The Dedicated Short Range Communication (DSRC) spectrum in $5.9 \mathrm{GHz}$ band consists of 7 channels [13]. Fig. A.1 shows the allocated channels and respective power allocation in DSRC spectrum. Out of 7 available channels, Channel 178, also known as the control channel $(\mathrm{CCH})$, is used to exchange high priority safety messages and other control information. Two channels at the edges are allocated for safety related applications and reserved for the future use. The remaining four channels, known as the service channels (SCHs), are 
allocateed for non-safety related applications. It is worth noting that vehicles negotiate in the $\mathrm{CCH}$ to determine which $\mathrm{SCH}$ to be tuned into for exchanging packets. Once the nodes negotiate, packets are exchanged in the negotiated $\mathrm{SCH}$.

In a VANET multi-channel environment, a set of packets that are associated with the safety and non-safety related applications and to be transmitted in SCHs is known as a service. A provider is a source node which offers a service and transmits associated packets to its neighboring nodes, referred to as users. A provider and users of a service negotiate in the $\mathrm{CCH}$ regarding the use of an $\mathrm{SCH}$ to transmit and receive the corresponding packets, respectively. The provider establishes a WAVE-mode Basic Service Set (WBSS) in a distributed manner by transmitting an announcement of the service through WAVE Service Advertisement (WSA). The announcement is performed in the $\mathrm{CCH}$, and the provider chooses an $\mathrm{SCH}$ where the service is to be provided. A user, if interested in the service, joins the WBSS established by the provider. In the wireless access in vehicular environment (WAVE)/IEEE 1609.4 standard [89], which governs the the multi-channel operations, the process of choosing an $\mathrm{SCH}$ to provide a service is not specified; however, it suggests that the provider should choose an $\mathrm{SCH}$ which has the least interference from its neighboring nodes (i.e., not used in the two-hop transmission distance of the provider) [90]. 


\section{Appendix B}

\section{Two-state Markov Channel}

Let the channel be either in good or bad state and remains unchanged in each time slot. In a new time slot, the channel either remains in the same state as in the previous time slot or alters to the other state. The channel is considered to be in the good state, if the received power $\gamma_{r}$, at the receiving node which is at distance $r$ meters from the transmitting node, is equal to or greater than a threshold value $\gamma_{t h}$; otherwise, the channel is considered to be in the bad state. Let random process $\left\{\mathcal{M}_{i}\right\}$ represents the channel state during time slots, $i=1,2,3, \ldots$. Hence, $\mathcal{M}_{i}$ is a two-state first-order Markov chain with transition probabilities $P_{g g}, 1-P_{g g}, 1-P_{b b}$ and $P_{b b}$, as shown in Fig. B.1. The transition probability, $P_{g g}$, can be written as

$$
\begin{aligned}
P_{g g} & =\operatorname{Pr}\left\{\mathcal{M}_{i+1}=\text { good } \mid \mathcal{M}_{i}=\text { good }\right\} \\
& =\frac{1-F_{\gamma_{r_{i}}}\left(\gamma_{t h}\right)-F_{\gamma_{r_{i+1}}}\left(\gamma_{t h}\right)+F_{\gamma_{r, 2}}\left(\gamma_{t h}, \gamma_{t h}\right)}{1-F_{\gamma_{r_{i}}}\left(\gamma_{t h}\right)}
\end{aligned}
$$

where $F_{\gamma, 2}(.,$.$) is the bivariate cumulative distribution function (cdf) and F_{\gamma_{r_{i}}}($.$) is the cdf$ of the amplitude of received signals. Similarly,

$$
\begin{aligned}
P_{b b} & =\operatorname{Pr}\left\{\mathcal{M}_{i+1}=b a d \mid \mathcal{M}_{i}=b a d\right\} \\
& =\frac{F_{\gamma, 2}\left(\gamma_{t h}, \gamma_{t h}\right)}{F_{\gamma_{r_{i}}}\left(\gamma_{t h}\right)} .
\end{aligned}
$$




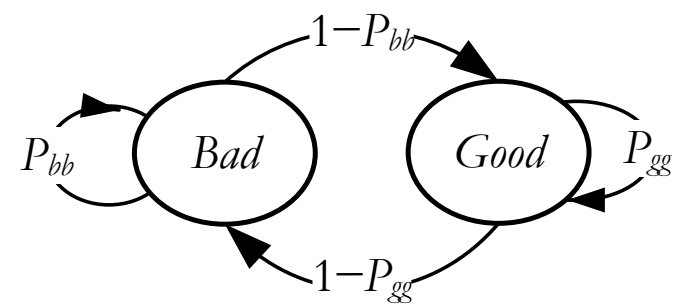

Figure B.1: Two-state Markov chain channel.

Consequently, the steady state probability of the channel to be in the good state, denoted as $\pi_{g}$, is given by

$$
\pi_{g}=\frac{1-P_{b b}}{2-P_{g g}-P_{b b}} .
$$

Transition probabilities in (B.1) and (B.2) depend on the bivariate cdf and cdf of the fading statistics. In the system, a generalized Nakagami- $m$ channel with correlated amplitudes is considered. For the Nakagami- $m$ channel, the corresponding cdf of the received power is given by

$$
F_{\gamma_{r}}(x)=1-\frac{\Gamma\left(m, m \frac{x}{\bar{\gamma}_{r}}\right)}{\Gamma(m)}
$$

where $\Gamma(\cdot, \cdot)$ is the upper incomplete Gamma function. Given two non-negative correlated Nakagami- $m$ random variables, say $X_{1}$ and $X_{2}$, the bivariate pdf is given by [91]

$$
\begin{aligned}
f_{X, 2}\left(x_{1}, x_{2} ; m, \varrho\right)= & \frac{4 m^{m+1}\left(x_{1} x_{2}\right)^{m}}{\mu_{1} \mu_{2}(1-\varrho)\left(\sqrt{\mu_{1} \mu_{2} \varrho}\right)^{m-1} \Gamma(m)} \\
& \times \exp \left\{-\frac{m}{1-\varrho}\left(\frac{x_{1}^{2}}{\mu_{1}}+\frac{x_{2}^{2}}{\mu_{2}}\right)\right\} \times I_{m-1}\left(\frac{2 m \sqrt{\varrho}}{\sqrt{\mu_{1} \mu_{1}}(1-\varrho)} x_{1} x_{2}\right)
\end{aligned}
$$

where

- $f_{X, 2}(\cdot, \cdot)$ is the bivariate pdf, 
- $\mu_{i}=E\left(X_{i}^{2}\right), i=\{1,2\}$,

- $I_{v}(\cdot)$ is the $v^{\text {th }}$ order modified Bessel function of the first kind, and

- $\varrho$ is the correlation coefficient.

For integer $m$ values, the corresponding cdf is given by [80]

$$
\begin{aligned}
F_{X, 2}\left(x_{1}, x_{2} ; m, \varrho\right) & =1-\sum_{k=0}^{m-1}\left[\exp \left(-\frac{m x_{2}^{2}}{\mu_{2}}\right)\left(\frac{m x_{2}^{2}}{\mu_{2}}\right)^{k} \frac{1}{k !}\right. \\
& +\left(\frac{m x_{1}^{2}}{\mu_{1}}\right)^{k} \frac{1}{k !}(1-\varrho)^{-k} \exp \left\{-\frac{m}{1-\varrho}\left(\frac{x_{1}^{2}}{\mu_{1}}+\frac{x_{2}^{2}}{\mu_{2}}\right)\right\} \\
& \times\left\{\left(\frac{m x_{2}^{2}}{\mu_{2}}\right)^{k} \frac{1}{k !} \Phi_{3}\left(1, k+1 ; \frac{x_{2}^{2}}{\mu_{2}} \frac{m}{(1-\varrho)}, \varrho\left(\frac{x_{1}}{\sqrt{\mu_{1}}} \frac{x_{2}}{\sqrt{\mu_{2}}} \frac{m}{(1-\varrho)}\right)^{2}\right)\right. \\
& \left.\left.-\sum_{i=1}^{m-k}\left(\frac{m x_{2}^{2}}{\mu_{2}}\right)^{k+i-1} \frac{1}{(k+i-1) !} \Phi_{3}\left(i, k+i ; \frac{x_{2}^{2}}{\mu_{2}} \frac{m \varrho}{(1-\varrho)}, \varrho\left(\frac{x_{1}}{\sqrt{\mu_{1}}} \frac{x_{2}}{\sqrt{\mu_{2}}} \frac{m}{(1-\varrho)}\right)^{2}\right)\right\}\right]
\end{aligned}
$$

where

- $F_{X, 2}(\cdot, \cdot)$ is the bivariate cdf,

- $\Phi_{3}(\cdot, \cdot ; \cdot, \cdot)$ is the confluent hypergeometric function, which can be approximated as

$$
\Phi_{3}(j, e ; \psi, z) \approx \sum_{k=0}^{2 m-1} \frac{(j)_{k} \Gamma(e)}{k !} \frac{\psi^{k}}{z^{(e+k-1) / 2}} I_{e+k-1}(2 \sqrt{z})
$$

- $(j)_{k}$ is the Pochhammer symbol [79], which is defined as

$$
(j)_{k}=j(j+1) \cdots(j+k-1), \quad(j)_{0}=1 \text { and } k=1,2, \cdots .
$$

From (B.4) and (B.6), the transition probabilities in (B.1) and (B.2) can be calculated and used to calculate the steady state probability in (B.3). The transition probabilities depend on the correlation coefficient of a received signal at two different time slots, which is given in (4.3). 